\title{
Advanced solid-state NMR spectroscopy of natural organic matter
}

Jingdong $\mathrm{Mao}^{1 *}$, Xiaoyan $\mathrm{Cao}^{2}$, Dan C. Olk ${ }^{3}$, Wenying $\mathrm{Chu}^{1}$, and Klaus Schmidt-Rohr ${ }^{2}$

${ }^{1}$ Department of Chemistry and Biochemistry, Old Dominion University, 4541 Hampton Blvd., Norfolk, VA 23529 (Jingdong Mao, e-mail: jmao@odu.edu; phone: 757-683-6874; fax: 757683-4628; Wenying Chu, e-mail: wxchu001@odu.edu)

${ }^{2}$ Department of Chemistry, Brandeis University, 415 South Street, Waltham, MA 02453

(Xiaoyan Cao, e-mail: xcao15@ brandeis.edu; phone: 781-736-2580; Klaus Schmidt-Rohr, email: srohr@brandeis.edu; phone: 781-736-2520; fax: 781-736-2516)

${ }^{3}$ National Laboratory for Agriculture and the Environment, 1015 N. University Blvd., Ames, IA 50011, USA. (Dan C. Olk, e-mail: dan.olk@ars.usda.gov; phone: 515-294-8412; fax: 515294-8125)

Submitted to Progress in Nuclear Magnetic Resonance Spectroscopy

*Author to whom correspondence should be addressed:

Jingdong Mao, e-mail: jmao@odu.edu; phone: 757-683-6874; fax: 757-683-4628

Number of pages: 137

Number of figures: 23

Number of tables: 0 


\section{Abstract}

Solid-state NMR is essential for the characterization of natural organic matter (NOM) and is gaining importance in geosciences and environmental sciences. This review is intended to highlight advanced solid-state NMR techniques, especially a systematic approach to NOM characterization, and their applications to the study of NOM. We discuss some basics of how to acquire high-quality and quantitative solid-state ${ }^{13} \mathrm{C}$ NMR spectra, and address some common technical mistakes that lead to unreliable spectra of NOM. The identification of specific functional groups in NOM, primarily based on ${ }^{13} \mathrm{C}$ spectral-editing techniques, is detailed and the theoretical background of some recently-developed spectral-editing techniques is provided. Applications of solid-state NMR to investigating nitrogen in NOM are described, focusing on limitations of the widely used ${ }^{15} \mathrm{~N}$ CP/MAS experiment and the potential of improved advanced NMR techniques for characterizing $\mathrm{N}$ forms in NOM. Then techniques used for identifying proximities, heterogeneities and domains are reviewed, and some examples provided. In addition, NMR techniques for studying segmental dynamics in NOM are reviewed. We also briefly discuss applications of solid-state NMR to NOM from various sources, including soil organic matter, aquatic organic matter, organic matter in atmospheric particulate matter, carbonaceous meteoritic organic matter, and fossil fuels. Finally, examples of NMR-based structural models and an outlook are provided.

Keywords: Natural organic matter; organic nitrogen; solid-state NMR; spectral-editing; domain 


\section{Contents}

1. Introduction

2. Basic considerations for acquiring high-quality, quantitative solid-state ${ }^{13} \mathrm{C}$ NMR spectra of natural organic matter (NOM)

2.1. High-quality spectra

2.1.1. Baseline distortion

2.1.2. Spinning sidebands

2.1.3. On-resonance decoupling

2.2. Quantitative spectra

2.3. Peak overlap

2.4. Observability and spin counting

3. Identification of functional groups

3.1. Introduction to spectral-editing techniques

3.2. Principles and practice of some recently developed spectral-editing techniques

3.2.1. ${ }^{13} \mathrm{C}$ chemical shift anisotropy filter

3.2.2. $\mathrm{CH}$ selection

3.2.3. $\mathrm{CH}_{2}$ selection

3.2.4. Recoupled ${ }^{1} \mathrm{H}-{ }^{13} \mathrm{C}$ long-range dipolar dephasing

3.2.5. Saturation-pulse induced dipolar exchange with recoupling (SPIDER)

3.3. Identification of specific functional groups

3.3.1. Ketones and aldehydes (220-185 ppm)

3.3.2. Carboxylic acids, carboxylates, esters, and amides (185-165 ppm)

3.3.3. Aromatic carbons bonded to heteroatoms (165-145 ppm) 
3.3.4. Other aromatics and olefinics (145-95 ppm)

3.3.5. Separation of anomeric from aromatic carbons (115-95 ppm)

3.3.6. O- and N-alkyl groups (112-52 ppm)

3.3.7. Nonpolar aliphatics (52-0 ppm)

4. Solid-state NMR techniques to investigate organic nitrogen in NOM

5. Connectivities and proximities

6. Heterogeneities and domains

6.1. Introduction to heterogeneities and domains

6.2. Techniques for identifying proximities, heterogeneities and domains

6.2.1. Goldman-Shen spin diffusion with ${ }^{13} \mathrm{C}$ detection

6.2.2. ${ }^{1} \mathrm{H}$ inversion recovery with ${ }^{13} \mathrm{C}$ detection

6.2.3. 2D HETCOR with spin diffusion

6.2.4. Combined rotation and multiple-pulse spectroscopy (CRAMPS) 2D exchange NMR

6.2.5. Long-range recoupled dipolar dephasing

6.2.6. Principles for selecting different techniques

6.3. Examples of close proximities, heterogeneities, and domains

6.3.1. Lignin residues

6.3.2. Fused-ring aromatic domains

6.3.3. Nonpolar aliphatic domains

7. Segmental dynamics

7.1. Importance of information on segmental dynamics

7.2. ${ }^{1} \mathrm{H}$ wideline spectra and wideline separation (WISE) 
7.3. Slow dynamics: Centerband-only detection of exchange (CODEX) NMR

7.4. Examples of segmental mobility in NOM - mobile lipids detected in NOM from natural waters

8. Applications of solid-state NMR to specific types of organic matter

8.1. Soil organic matter

8.2. Aquatic organic matter

8.2.1. Solid-state ${ }^{13} \mathrm{C}$ NMR applications

8.2.2. Solid-state ${ }^{15} \mathrm{~N}$ NMR applications

8.2.3. Solid-state ${ }^{31} \mathrm{P}$ NMR applications

8.3. Organic matter in atmospheric particulate matter

8.4. Organic matter in carbonaceous meteorites

8.5. Fossil fuels

9. Structural models based on NMR-derived structural information

10. Summary and outlook

Acknowledgments

References 


\section{Introduction}

Abundant nonliving natural organic compounds exist in soil, water, and sediment [1-6]. The generic term for these organic molecules is natural organic matter (NOM). NOM can be involved in agricultural, environmental, geochemical, and energy issues by playing important roles in fundamental processes. For example, in agriculture it controls many physical, chemical and biological processes in soils, such as enhancing soil fertility and improving soil structure [1]. In the environment, it regulates the fate and transport of inorganic and organic pollutants, and thus influences their toxicity [7.] In addition NOM functions as both a sink and source in the global carbon and nitrogen cycles [8]. Fossil fuels such as coal and oil shale still provide a significant proportion of the energy consumed worldwide $[9,10]$. Furthermore, there is intense research interest in converting biomass to biorenewable energy products [11].

The first step towards understanding NOM reactivity, properties and functions is to identify its composition and functional groups, which is extremely challenging though, due to the structural complexity and heterogeneity of NOM. Decades of research have shown that NOM is a heterogeneous mixture of various functional units present in charged macromolecules of polydisperse size $[4,12]$. These functional units include nonpolar alkyl, carbohydrate-like, protein-like, lignin-like, heterocyclic, and polycyclic aromatic moieties. One new paradigm considers soil NOM as a heterogeneous mixture of physical states with a hierarchy of preferred sites [13]. Although several more hypotheses on NOM structures have been proposed, none of them has been confirmed at the molecular level. Various methods have been used to investigate NOM structure, including chemical degradation, thermal degradation and spectroscopic methods $[6,14,15]$. Although chemical and thermal degradation methods could yield structural information on subunits, the structures of these subunits might be 
unrepresentative, or even from artifacts, and difficult to relate directly back to the original structures of NOM. Many spectroscopic methods such as infrared (IR), electron paramagnetic resonance, Raman, fluorescence and nuclear magnetic resonance (NMR) spectroscopies have been applied to NOM [1], and it has been established that non-destructive spectroscopic analyses such as NMR spectroscopy are better choices than destructive approaches [16]. Compared to other spectroscopic methods such as IR or Raman spectrocopy, solid-state NMR allows for comprehensive and quantitative structural information to be obtained from NOM, and is therefore arguably one of the most powerful techniques for its comprehensive characterization.

Both solid- and liquid-state NMR spectroscopy have been extensively used to investigate NOM structure, and have been the topic of several recent excellent reviews [17-22]. Solution NMR is very useful for studying soluble components in NOM, especially those with low molecular weight. However, compared with solid-state NMR, solution NMR has some disadvantages when applied to NOM: 1) Some NOM samples, or some fractions of NOM, are not soluble; 2) solid-state NMR facilitates a much higher sample concentration than solution NMR, enhancing signals and saving instrument time; making the NOM concentration high enough to achieve a strong signal in the solution state may lead to aggregation, resulting in lower sensitivity, lower resolution, and loss of structural information; 3) solid-state NMR generally requires less sample handling, and is free from solvent effects that may introduce artifact peaks; 4) solid-state NMR is conservative, i.e., it does not consume sample. Solution NMR is not conservative; changes that occur during sample preparation and analysis by solution NMR would void further analyses by other methods; 5) it is much easier and more straightforward to detect nonprotonated carbons using solid-state NMR techniques, while most 
advanced modern solution NMR techniques employ ${ }^{1} \mathrm{H}$ detection, greatly overemphasizing, in NOM, the signals of protonated sites; 6) in solution NMR, the fast tumbling of molecules averages anisotropic interactions, while in solid-state NMR these anisotropic interactions can be manipulated with specially developed pulse sequences to extract structural information not available from solution NMR; 7) solid-state techniques can identify domains and heterogeneities within NOM structures, while solution NMR cannot; and 8) the macromolecular structures, aggregates and colloids of NOM slow the tumbling of these molecules, leading to $\mathrm{T}_{2}$ values that are too short to allow many of the pulse sequences of solution NMR to be successfully used. Based on experience with pure organic compounds, one probably has an intuitive notion that solution NMR spectra of complex NOM are of higher resolution than the corresponding solid-state NMR spectra, and that high magnetic fields provide better resolution than lower magnetic fields in solution NMR. However, this does not hold true for NOM samples with inhomogeneous line broadening. Given the aforementioned limitations associated with solution NMR when applied to NOM, solution NMR spectra of NOM should be interpreted with caution; for instance, one should not attempt a complete NOM picture based on partial structures mostly of low-molecular weight and protonated species derived from solution NMR.

Solid-state NMR has been widely employed to study NOM structure, with most studies relying on the routine, at best semi-quantitative ${ }^{13} \mathrm{C}$ cross polarization/magic angle spinning (CP/MAS) technique. This technique has some known limitations, such as non-quantitative peak intensities [23]. The literature indicates additional problems, such as spinning sidebands, baseline distortion, and ambiguous assignments. Recently a number of multi-dimensional techniques and advanced spectral-editing approaches have been applied with great promise for 
systematically characterizing complex NOM in plants, soils, water, sediments, as well as in the Murchison meteorite [24], and have provided substantial additional information over basic CP/MAS studies alone. Routine ${ }^{13} \mathrm{C}$ solid-state NMR spectra consist of broad and heavily overlapped bands in which functional groups cannot be clearly distinguished. This limitation can generally be overcome by using some spectral-editing techniques that selectively retain certain peaks and eliminate others, clearly revealing specific functional groups. Twodimensional ${ }^{1} \mathrm{H}_{-}{ }^{13} \mathrm{C}$ heteronuclear correlation NMR can be used to identify through-bond connectivity or spatial proximity of different specific functional groups. In addition, ${ }^{1} \mathrm{H}$ spin diffusion can be adopted to detect domains or heterogeneities on a 1- to 50-nm scale, as a basis for realistic structural models of different forms of NOM in the environment. Furthermore, solid-state NMR techniques enable detailed investigations of dynamics over a wide range of motional rates $[25,26]$. Application of these advanced NMR techniques has enabled the identification of aliphatic domains, char domains, lignin-derived regions, peptide-sugar polymers, as well as indications of linked aromatic and sugar rings in NOM. Finally, quantitative structural information can be obtained using direct polarization/magic angle spinning (DP/MAS) and DP/MAS with recoupled dipolar dephasing or multiple cross polarization/MAS (multiCP/MAS) and multiCP with dipolar dephasing [27].

This review is intended to highlight advanced solid-state NMR techniques and their applications to the study of NOM. Section 2 discusses some basics of how to acquire highquality, quantitative solid-state ${ }^{13} \mathrm{C}$ NMR spectra, and lists some common technical mistakes that lead to unreliable spectra of NOM. Section 3 details the identification of specific functional groups in NOM, primarily based on spectral-editing techniques. In Section 4, the applications of solid-state NMR to investigating nitrogen in NOM are described. 
Connectivities and proximities are dealt with in Section 5. Section 6 discusses heterogeneity and domains within NOM, as investigated by solid-state NMR techniques such as GoldmanShen spin diffusion. Segmental dynamics within NOM, which are important for addressing the sorption of organic contaminants, are described in Section 7. In Section 8, we review reported applications of advanced solid-state NMR techniques to study NOM from various sources. Section 9 introduces structural models that are based on NMR-derived structural information. The concluding Section 10 presents a summary as well as an outlook for future research in studies of NOM using NMR spectroscopy.

\section{Basic considerations for acquiring high-quality, quantitative solid-state ${ }^{13} \mathrm{C}$ NMR spectra of natural organic matter (NOM)}

Solid-state NMR has been applied to the study of NOM for several decades (see for example, $[11,15,18,19,22,28-35])$. Nevertheless, the acquisition of high-quality, quantitative

${ }^{13} \mathrm{C}$ spectra of NOM is still not routine for this field, due to technical and access limitations. The most common problems are spinning sidebands and baseline distortion resulting from spectrometer dead-time after single-pulse excitation. These issues have become more prominent with the increase of commercially available magnetic field strengths $B_{0}$, as discussed below. Therefore, it is necessary first to address basic principles for acquiring highquality, quantitative solid-state NMR spectra of NOM. Without taking these into account, interpretation of solid-state NMR spectra from complex NOM would be unreliable, because the collection of complex NMR spectra containing broad bands is different from that for pure organic compounds that typically give rise to spectra with sharp lines. NMR spectra of NOM have often been acquired using the solution NMR approach for well-resolved spectra, which 
will cause baseline distortion in the case of broad-line NOM spectra. For example, as shown below, for acquisition of broad NMR spectra of NOM, one needs to insert a Hahn echo before detection in order to avoid baseline distortion. An alternative would be to optimize the resonant circuit to minimize baseline problems.

\subsection{High-quality spectra}

Several problems can interfere with the acquisition of high-quality solid-state ${ }^{13} \mathrm{C} N \mathrm{NR}$ spectra of NOM. Here we focus on baseline distortion, sidebands, and on-resonance decoupling. Several precautions that should be taken are described.

\subsubsection{Baseline distortion}

Almost all reported solid-state ${ }^{13} \mathrm{C}$ NMR spectra of $\mathrm{NOM}$ in the literature contain characteristically broad lines (Fig. 1), primarily due to the presence of potentially thousands of related but different chemical species in NOM samples. The baselines of some published ${ }^{13} \mathrm{C}$ CP/MAS spectra of NOM are significantly distorted, due to the dead time at the start of detection [26, 36], which arises from finite pulse ring-down and filter/receiver response times.

Based on Fourier transform theory, the free induction decay (FID) associated with the broad lines of NOM samples decays very rapidly. Due to pulse ring-down in the probe coil, detection should be started after the "dead time", which is the time period between the end of the pulse and the time at which the energy of the pulse has been sufficiently dissipated that the receiver can be gated on and NMR signal detected. However, during the dead time the FID is already evolving, which means that the first portion of the FID cannot be collected. This poses a serious problem to NOM with its rapidly decaying signals, because the FID lost during the 
dead time accounts for a significant part of the total FID, leading not only to loss of spectral intensity, but also to spectral distortions. Specifically, transverse magnetization evolves under nuclear spin interactions during the dead time, and the isochromats with their different precession frequencies gradually dephase. Therefore, when an FID is recorded only starting after the dead time, different isochromats have acquired different initial phases. Fourier transformation generates severely distorted lineshapes because there is a wide range of phases of transverse magnetization when acquisition starts late for broad NOM signals. In solution NMR of pure compounds, the FID of samples with resolved peaks decays much more slowly and therefore the signal loss is negligible during the dead time. In this case, the phase problem due to the dead time can be nearly eliminated by a first-order phase correction. However, in solid-state NMR of NOM or even solution NMR of NOM where FID decay is much faster and a significant fraction of the signal is lost during the dead time, a first-order phase correction cannot correct the dead-time problem but rather results in serious baseline distortions [26, 36].

\section{Caution 1: Avoid using a first-order phase correction for broad NOM spectra.}

It has been well established that a Hahn echo, $\tau-\pi$-pulse $-\tau$, inserted before detection in pulse sequences can overcome this baseline distortion due to dead time $[26,36]$. However, under MAS, the duration of $\tau$ has to follow some principles. For example, if a $\pi$ pulse is placed close to the middle of the rotation period, the magnetization of sites with significant chemical-shift anisotropy is dephased instead of being refocused [37]. There are two approaches to determine the optimum value of $\tau$. The first is to set $\tau$ equal to a rotation period $\mathrm{t}_{\mathrm{r}}$ (or a multiple thereof). In principle, ${ }^{13} \mathrm{C} \mathrm{T}_{2}$ relaxation reduces signals during the time $2 \tau=2 \mathrm{t}_{\mathrm{r}}$ before detection. In practice, in the case of fast spinning with short rotation periods the ${ }^{13} \mathrm{C} \mathrm{T}_{2}$ 
relaxation effect is negligible. The ${ }^{13} \mathrm{C} \mathrm{T}_{2}$ values of solid samples of humic acids are usually on the order of a few milliseconds. This allows for the use of $2 \tau=2 t_{\mathrm{r}}=143 \mu$ s (at a spinning frequency of $14 \mathrm{kHz}$ ) without significant spectral distortions. The second approach is to use a very short $\tau$ (for example $8 \mu \mathrm{s}$ ), so that the difference in the magnitude of any phase difference acquired by a given isochromat in either of the two $\tau$ periods is small. In the case of low spinning frequencies, during the two $\tau$ periods the segments and interaction tensors change their orientations to an insignificant extent and a quasi-static approximation can be used. But for fast spinning, $\tau$ must be short $(2 \tau<20 \mu \mathrm{s})$ so that the precession phases remain proportionally small, with the result that the MAS effects are of second order. The optimum phases for the $\pi$-pulse are different for the two approaches [38]: With a short $\tau$, the $\pi$-pulse should be applied along the initial magnetization. If $\tau$ is $>20 \mu$ s, the EXORCYCLE scheme should be used, which cycles the phase of the $\pi$-pulse through $x, y,-x,-y$ and inverts the receiver phase with each $90^{\circ}$ shift in the pulse phase [39].

The echo maximum does not necessarily occur exactly at the time $2 \tau$, due to the effects of finite pulse length and finite probe bandwidth, which lead to linear phase distortions that correspond to a shift of the echo maximum in the time domain. To adjust the timing of the start of detection and achieve a perfect baseline, a sample with very good sensitivity, such as a ${ }^{13} \mathrm{C}$ labeled model compound with sharp peaks over the full spectral range ( 30-180 ppm), should be used. In the authors' laboratories, a mixture of three amino acids: ${ }^{13} \mathrm{CH}_{3}$-labelled alanine, ${ }^{13} \mathrm{CH}_{2}$-labelled glycine, and ${ }^{13} \mathrm{COO}$-labelled leucine is routinely used for this purpose. For DP/MAS spectra with a $14 \mathrm{kHz}$ spinning frequency, timings that are mis-set by as little as $2 \mu \mathrm{s}$ can result in significant spectral distortions. In practice, the first part of echo (the first $\tau$ ) in the pulse sequence is fixed, and then the duration of the second part of echo is adjusted until a very 
good baseline is obtained. Figure 2 demonstrates this for the mixture of three amino acids. Figure 2. (a) is the Hahn-echo pulse sequence used. With a well-set Hahn echo, Fig. 2(b) shows a perfect flat baseline. However, if the signal is detected $5 \mu$ s too early (Fig. 2(c)), the baseline on either end of the spectrum will bend down. In this case, the duration of the second delay before the echo needs to be prolonged to achieve a flat baseline as in Fig. 2(b). In contrast, if the magnetization is detected $5 \mu$ s too late (Fig.2(d)), the baseline beyond the peak regions will bend up; then the length of the second part of the echo needs to be shortened. Once the ideal timings have been set on the model compound, they can be used to obtain NOM spectra with good baselines, using only constant phase correction (without linear/first-order correction). An 'echo sequence' before detection is also obligatory in more complicated pulse sequences used for investigating NOM.

Finally, we use a commercial NOM, the International Humic Substances Society (IHSS) peat humic acid (HA) (Fig. 3), to demonstrate the correct set-up of the Hahn echo for NOM spectra. Figure 3(a) is the undistorted ${ }^{13} \mathrm{C}$ DP/MAS spectrum of this material with a good baseline, allowing reliable quantitative structural information to be obtained. Figures 3(b) and (c) display the spectra obtained with the acquisition started $5 \mu$ s and $15 \mu$ s too early, respectively. The phenomenon of "bending down of the baseline" is evident. Figures $3(\mathrm{~d})$ and (e) show the spectra with the acquisition started $5 \mu$ s and $15 \mu \mathrm{s}$, respectively, after the echo maximum. The phenomenon of "bending up of the baseline" is clearly evident for the $15 \mu \mathrm{s}$ delayed acquisition (Fig. 3(e)). Serious spectral distortions occur when an echo is not used before detection (Fig. 3(f)), which is the case for many spectra in the NOM literature. It is clear that the lineshape of the spectrum acquired without a Hahn echo is significantly different from that in Fig. 3(a). In order to obtain an "improved" baseline, a first-order baseline correction 
was often applied to spectra in the literature, as shown in Fig. 3(g), but this causes distorted lineshapes despite an apparently "good" baseline. Thus, in order to obtain a quantitative ${ }^{13} \mathrm{C}$ DP/MAS spectrum, (1) refocusing magnetization using a Hahn echo is absolutely necessary; (2) first-order baseline correction must be avoided; and (3) the timing for a perfect start of detection at the echo maximum should be determined using a model compound.

\section{Caution 2: Magnetization must be refocused before detection.}

\subsubsection{Spinning sidebands}

Under magic-angle spinning at a frequency $v_{\mathrm{r}}$ that is comparable to the chemical-shift anisotropy (CSA), pronounced spinning sidebands are observed at $\pm v_{\mathrm{r}}$ (and others with lower intensity at higher multiples of $v_{\mathrm{r}}$ ). The largest CSAs observed in NOM, for aromatic carbons, spread over about $\pm 100 \mathrm{ppm}$, corresponding to $\pm 10 \mathrm{kHz}$ in a 9.4-T field; the spinning frequency needs to significantly exceed $10 \mathrm{kHz}$ for sidebands to be insignificant. Some spectra in the literature are afflicted with serious sidebands, which make even qualitative conclusions difficult, let alone quantitative determinations. Sidebands reduce the intensity of centerbands, leading to the loss of intensity and distortion of peak areas. More seriously, the aromatic sidebands in spectra of NOM with a large aromatic fraction can overlap with aliphatic signals, making even a qualitative analysis impossible. For example, for a ${ }^{13} \mathrm{C} C \mathrm{CP} / \mathrm{MAS}$ spectrum acquired at a spinning frequency of $5 \mathrm{kHz}$ and a ${ }^{13} \mathrm{C}$ frequency of $100 \mathrm{MHz}$, the ${ }^{13} \mathrm{C}$ sidebands are separated from their centerbands by only $50 \mathrm{ppm}$. Therefore, the strong first-order sidebands of aromatic signals between 120-140 ppm overlap with the aliphatic signals between 70-90 ppm. Under this condition, the sidebands of aromatics embedded in the signals of aliphatics can lead to an overestimate of aliphatic and an underestimate of aromatic intensities. 
There are two common approaches used to remove or reduce sidebands, namely (i) spinning samples fast and (ii) inserting a sequence of total suppression of sidebands (TOSS) before detection [40]. Sufficiently fast MAS can be achieved only with relatively small rotors, which reduces the sensitivity because less sample material can be packed in small rotors. In a 400-MHz spectrometer, i.e., at a field of $9.4 \mathrm{~T}$, spinning at $14 \mathrm{kHz}$ with $4-\mathrm{mm}$ rotors moves sidebands out of the centerband region and makes them almost negligible. At higher fields, proportionally faster spinning is required to achieve this favorable situation [36, 41]. Currently, spinning frequencies up to $111 \mathrm{kHz}$ are accessible on commercial instruments, but again at the expense of sample volume and signal.

At low spinning frequencies, the insertion of four- $\pi$-pulse TOSS [40] before detection can remove sidebands. However, with the TOSS sequence signals of $\mathrm{sp}^{2}$-hybridized carbons are less completely refocused because of their larger CSA than are $\mathrm{sp}^{3}$-hybridized carbon signals. Therefore, $\mathrm{sp}^{2}$-hybridized carbons are underestimated and $\mathrm{sp}^{3}$-hybridized carbons overestimated. Also, ${ }^{13} \mathrm{C} \mathrm{T}_{2}$ relaxation occurs during the four TOSS $\pi$-pulses, in particularly for $\mathrm{CH}_{2}$ groups. Thus, TOSS spectra are not quantitative. The TOSS sequence can be implemented using a sample with good sensitivity such as a ${ }^{13} \mathrm{COO}-$ labeled glycine or a model lignin. Since the TOSS $\pi$-pulses generate an echo, a good baseline of a ${ }^{13} \mathrm{C} \mathrm{CP} / \mathrm{TOSS}$ spectrum can be achieved, similar to the set-up of the Hahn echo, by adjusting the delay after the last $\pi$ pulse in the TOSS sequence and before detection. The baseline patterns of "detection too early" and "detection too late" are the same as in Fig. 2 and Fig. 3 and can be corrected by prolonging or shortening the last delay before detection until a perfect baseline is achieved. Sometimes, residual sidebands can still be observed in a TOSS spectrum due to imperfect $\pi$ pulse length; varying the $\pi$ pulse length slightly can usually suppress such residual sidebands. 


\subsubsection{On-resonance decoupling}

In solid-state NMR, one of the essential techniques to obtain well-resolved spectra is heteronuclear dipolar decoupling of protons. For several decades, high-power continuous-wave (CW) decoupling was the most common decoupling technique [26, 41]. However, CW decoupling only achieves a zero-order approximation to a full decoupling system under MAS. Under MAS, anisotropic heteronuclear dipolar couplings are time-dependent; moreover the radiofrequency irradiation also imposes a time dependence on the spin system. There are interference effects between these two time-dependent processes if they occur on the same timescale [42]. The most important interference effects occur under either the condition $n \omega_{\mathrm{r}}=$ $\omega_{1}$ (with $\mathrm{n}=1,2 ; \omega_{\mathrm{r}}$ the spinning frequency; and $\omega_{1} \propto B_{1}$ the decoupling strength), which leads to a recoupling of heteronuclear dipolar couplings, or the condition $1 / 2 \omega_{\mathrm{r}}=\omega_{1}$, which results in a recoupling of homonuclear dipolar interactions. Thus, efficient decoupling cannot be achieved if the two time-dependent processes occur on the same timescale. Two-pulse phasemodulated decoupling (TPPM) [43] provides significant improvements in line width and intensity, which is due to the reduced second-order cross-term between the chemical shift of the irradiated spins and the heteronuclear dipolar coupling [44-46]. This high-power multiplepulse decoupling consists of two pulses with nutation angles of about $165-180^{\circ}$ and a phase difference between the two pulses of $10-50^{\circ}$. Figure 4 shows the ${ }^{13} \mathrm{C}$ spectrum of cholesterol under different decoupling conditions and two spinning frequencies $(6.5 \mathrm{kHz}$, left column; 14 $\mathrm{kHz}$, right column). Figures 4 (a)-(h) and (i)-(p) are spectra obtained with $\mathrm{CW}$ decoupling and TPPM decoupling, respectively. Figure 4 demonstrates several points concerning decoupling

quality: for all the decoupling powers shown, use of the higher spinning frequency of $14 \mathrm{kHz}$ 
leads to poorer decoupling relative to that with the lower spinning frequency of $6.5 \mathrm{kHz}$, and the linewidths under CW decoupling are larger than those with TPPM decoupling. Also, the increase of decoupling power level from 48, 54 to $61 \mathrm{kHz}$ progressively improves the decoupling, while applying the decoupling field off-resonance leads to poorer decoupling than if it is applied on-resonance.

\subsection{Quantitative spectra}

Although CP can enhance sensitivity and also shorten recycle delays, several problems such as low CP efficiency of nonprotonated and mobile carbons or regions having short $\mathrm{T}_{1 \rho}{ }^{\mathrm{H}}$ (proton rotating-frame spin-lattice relaxation time), spinning sidebands, and baseline distortions can make ${ }^{13} \mathrm{C}$ CP/MAS spectra non-quantitative. These problems can be solved by using the ${ }^{13} \mathrm{C}$ DP/MAS technique. In the DP/MAS, direct polarization is used, and thus the problems associated with $\mathrm{CP}$ are avoided. Moreover, unlike CP/MAS, DP/MAS remains insensitive to rf-field inhomogeneity even at high spinning frequencies where the sidebands are small and outside the region of the centerbands; therefore, it is easy to integrate the small sidebands and add their intensities to that of the centerband. In our case, at a spinning frequency of $14 \mathrm{kHz}$ the total sidebands of the aromatic and aromatic C-O groups are reduced to $10 \%$ and $6.4 \%$ of the centerbands, respectively. Furthermore, as previously discussed, with the introduction of a rotation-synchronized Hahn echo before detection, baseline distortion can be avoided.

However, the major obstacle to more widespread use of DP in studies of NOM is its reduced sensitivity and long recycle delays relative to $\mathrm{CP}$. The choice of the recycle delay of a

${ }^{13} \mathrm{C}$ DP/MAS experiment is controlled by ${ }^{13} \mathrm{C} \mathrm{T}_{1}$ values, not by ${ }^{1} \mathrm{H}_{\mathrm{T}}$ values as in the case of 
${ }^{13} \mathrm{C} \mathrm{CP} / \mathrm{MAS}$. Usually, ${ }^{13} \mathrm{C} \mathrm{T}_{1}$ values are much longer than the corresponding ${ }^{1} \mathrm{H} \mathrm{T}_{1}$ values, due to efficient ${ }^{1} \mathrm{H}$ spin diffusion from fast-relaxing sites such as rotating $\mathrm{CH}_{3}$ groups. In order to acquire a quantitative ${ }^{13} \mathrm{C}$ DP/MAS spectrum, the recycle delay must be four to five times longer than the longest ${ }^{13} \mathrm{C} \mathrm{T}_{1}$ value in an NOM sample. This suggests that the acquisition of a quantitative ${ }^{13} \mathrm{C}$ DP/MAS spectrum can be very time-consuming. However, ${ }^{13} \mathrm{C} \mathrm{T}_{1}$ relaxation times of NOM measured by the $\mathrm{CP} / \mathrm{T}_{1}$ method $[47,48]$ are not too dauntingly long, probably due to the effects of some unpaired electrons. Recycle delays required for most of the NOM samples studied by the authors are within 50 s, and almost all within 200 s. Around 1024 scans are often sufficient to obtain a quantitative spectrum with acceptable signal-to-noise ratio $(\mathrm{S} / \mathrm{N})$. This indicates that a ${ }^{13} \mathrm{C}$ DP/MAS spectrum with reasonable $\mathrm{S} / \mathrm{N}$ can be obtained within 15 hrs. Considering the quantitative reliability of ${ }^{13} \mathrm{C}$ DP/MAS spectra and unreliability of ${ }^{13} \mathrm{C}$ $\mathrm{CP} / \mathrm{MAS}$, it is absolutely worthwhile to employ ${ }^{13} \mathrm{C}$ DP/MAS if quantitative structural information is needed. Moreover, ${ }^{13} \mathrm{C} \mathrm{CP} / \mathrm{MAS}$ spectra are significantly distorted for NOM samples with abundant nonprotonated carbons, for instance from soils containing significant char residues [49] and with significant mobile groups such as some marine sinking particles [50], or for 'dirty' samples [51] such as insoluble organic matter from carbonaceous meteorites containing significant $\mathrm{Fe}$ [52]. In these cases, ${ }^{13} \mathrm{C}$ DP/MAS is more reliable and thus deemed necessary. For some dirty samples such as humin [53] and insoluble organic matter from carbonaceous meteorites [52], unpaired electrons in ferromagnetic and paramagnetic components spread the ${ }^{1} \mathrm{H}$ resonance frequencies over a wide range and thus prevent onresonance ${ }^{1} \mathrm{H}$ decoupling $[52,53]$. At standard spinning frequencies, this can make a significant fraction of $\mathrm{CH}_{2}$ and some $\mathrm{CH}$ signals too broad to detect. For these samples, the 
anisotropic couplings to the unpaired electrons can be partially "spun out" by using high spinning frequencies such as 30-kHz MAS [54].

At present, CP/MAS is still regarded as a quantitative technique by some researchers in the NOM community. In order to demonstrate that this is not the case, here we show the prominent difference between CP and DP spectra of NOM samples (Fig. 5). Figure 5(a) compares the DP and CP spectra of a Mollisol humic acid, which contains a large aromatic fraction. It is obvious that CP/TOSS overestimates $\mathrm{sp}^{3}$-hybridized carbons (0-90 ppm). Figure 5(b) shows DP and CP spectra of a humic acid from water in the St. Lawrence Estuary. A detailed study [55] found that this humic acid contained significant, highly mobile nonpolar aliphatics. These highly mobile nonpolar aliphatics are only inefficiently excited by $\mathrm{CP}$. Thus, comparing the $\mathrm{CP}$ and DP spectra, some features comprising narrow peaks at 15, 24, and $31 \mathrm{ppm}$ present in the DP spectrum disappear in the CP spectrum because of their high mobility. Figure 5(c) shows DP and CP spectra of a commercial 4-nm nanodiamond. Significant differences exist between these DP and CP spectra; CP selects carbons only on the diamond surface, failing to excite carbons in the diamond core; DP accurately reflects the whole structure of this nanodiamond [56]. These examples demonstrate that DP is more reliable for quantitation than is $\mathrm{CP}$.

\section{Caution 3: CP spectra are at best semi-quantitative.}

Since it is necessary to set the relaxation delay 4-5 times longer than the longest ${ }^{13} \mathrm{C} \mathrm{T}_{1}$ in an NOM sample in order to obtain a quantitative DP/MAS spectrum, one needs to know the

${ }^{13} \mathrm{C}_{1}$ in order to determine the recycle delay required to ensure that all the carbon sites are fully relaxed. Choosing a very long recycle delay, for instance $1000 \mathrm{~s}$, is usually not a workable solution, since then the number of scans that can be averaged within the given 
experiment time will be too small and the resulting spectrum too noisy. Very often in published studies the recycle delays used when acquiring quantitative ${ }^{13} \mathrm{C}$ DP/MAS spectra of NOM were not optimized. However, suitable recycle delays for quantitative ${ }^{13} \mathrm{C}$ DP/MAS spectra can be determined by the $\mathrm{CP} / \mathrm{T}_{1} / \mathrm{TOSS}$ technique [47]. In this technique, after cross-polarization from ${ }^{1} \mathrm{H}$ and before the TOSS $\pi$-pulse train, $\mathrm{a}+\mathrm{z} / \mathrm{-z}$ filter is employed so that the signal decays from full intensity to zero as a result of ${ }^{13} \mathrm{C}_{1}$ relaxation and phase cycling [57].

Figure 6 explains the working principle of the $\mathrm{CP} / \mathrm{T}_{1}$ method $[47,48]$. The storage pulse in alternate scans stores the magnetization along $+\mathrm{z} / \mathrm{-}$ and the receiver phase is correspondingly alternated (+/-). Magnetization stored along $+\mathrm{z}$ as shown in Fig. 6(a) - (c) equilibrates following equation (1):

$$
M\left(t_{z}\right)=(M(0)-M(\infty)) \exp \left(-t_{z} / T_{1}\right)+M(\infty)
$$

while magnetization stored along $-\mathrm{Z}$ as depicted in Fig. 6(d) - (f) follows the analogous equation (2):

$$
M\left(t_{\mathrm{z}}\right)=\left(M^{\prime}(0)-M(\infty)\right) \exp \left(-t_{\mathrm{z}} / T_{1}\right)+M(\infty)
$$

The subtraction of magnetization between successive scans that is achieved by the $+/-$ alternation of the receiver results in:

$$
M_{2 \text { scans }}\left(t_{\mathrm{z}}\right)=\left(M(0)-M^{\prime}(0)\right) \exp \left(-t_{z^{\prime}} / T_{1}\right)=2 M(0) \exp \left(-t_{z^{\prime}} / T_{1}\right)
$$

where in the last step we have used that $M^{\prime}(0)=-M(0)$. Thus, the recorded magnetization exponentially decays with a time constant of $T_{1}$.

In practice, the duration of the $z$-period $t_{\mathrm{z}}$ is increased, typically from $1 \mathrm{~ms}$ through $5 \mathrm{~s}, 20$ s, $40 \mathrm{~s}, 60 \mathrm{~s}, 100 \mathrm{~s}$, until eventually the residual intensity decreases to less than $5 \%$. The reference spectrum with the z-period $\mathrm{t}_{\mathrm{z}}$ of $1 \mathrm{~ms}$, which requires little time per scan, is typically measured with 512 scans, while the relaxed magnetization is recorded with 64 scans and scaled 
up by a factor of $512 / 64=8$ relative to the reference [47]. An important advantage of the $\mathrm{CP} / \mathrm{T}_{1} / \mathrm{TOSS}$ experiment over inversion- or saturation-recovery experiments is the knowledge of the value of the magnetization after very long delays, which is zero. Usually, in the authors' laboratories this experiment has been performed with larger sample rotors at a lower spinning frequency of 5 or $6 \mathrm{kHz}$ in order to increase the sensitivity and thus reduce the numbers of scans needed; most recently, magnetization has instead been generated by multiCP, in a multiCP/T 1 experiment at a $14-\mathrm{kHz}$ spinning frequency.

We also need to discuss the technique of ramped $\mathrm{CP}$, which has been highly recommended in the literature for the characterization of NOM as a reliable, quantitative technique that can potentially replace DP $[38,58-62]$. In order to conduct conventional CP/MAS experiments, proton and carbon radio-frequency field strengths $B_{1, \mathrm{H}}$ and $B_{1, \mathrm{C}}$, respectively, are adjusted so that the Hartmann-Hahn condition $\gamma_{\mathrm{H}} B_{1, \mathrm{H}}=\gamma_{\mathrm{C}} B_{1, \mathrm{C}}$ is met, where $\gamma_{\mathrm{H}}$ and $\gamma_{\mathrm{C}}$ are the gyromagnetic ratios of ${ }^{1} \mathrm{H}$ and ${ }^{13} \mathrm{C}$, respectively. At low spinning frequencies, matching profiles are centered on the Hartmann-Hahn condition and broad, making cross polarization easy to achieve even in the presence of $B_{1}$ inhomogeneity. However, for faster MAS, the Hartmann-Hahn matching profiles split into a series of 5 narrow bands separated by the spinning frequency $\omega_{\mathrm{r}}$ :

$$
\gamma_{\mathrm{H}} B_{1, \mathrm{H}}=\gamma_{\mathrm{C}} B_{1, \mathrm{C}}+n \omega \mathrm{r}, \quad \text { with } n=0, \pm 1, \pm 2
$$

The $\mathrm{CP}$ rate at the centerband $(n=0)$, which corresponds to the exact Hartmann-Hahn condition, is smaller than that at the sidebands. However, the matching condition at the sidebands $(n \neq 0)$ is sensitive to $B_{1}$ inhomogeneity: For instance, $\gamma_{\mathrm{H}} B_{1, \mathrm{H}}=2 \pi 60 \mathrm{kHz}$ and $\gamma_{\mathrm{C}}$ 
$B_{1, \mathrm{C}}=2 \pi 46 \mathrm{kHz}$ in the center of the rf coil at $\omega_{\mathrm{r}}=2 \pi 14 \mathrm{kHz}$ fulfills the matching condition. Near the end of the coil, where the fields are only half as strong, we have $\gamma_{\mathrm{H}} B_{1, \mathrm{H}}=2 \pi 30 \mathrm{kHz}$ and $\gamma_{\mathrm{C}} B_{1, \mathrm{C}}=2 \pi 23 \mathrm{kHz}$, so their difference is only $2 \pi 7 \mathrm{kHz}$ and the matching condition is not fulfilled. To achieve the Hartmann-Hahn match throughout the sample, an amplitude ramp is introduced on either of the radio-frequency channels $\left({ }^{1} \mathrm{H}\right.$ or $\left.{ }^{13} \mathrm{C}\right)$ during the contact time, sweeping one of the first-order sidebands $(n= \pm 1)$ in the Hartmann-Hahn profile $[58,59]$. This ramped $\mathrm{CP}$ has significantly improved the performance of $\mathrm{CP}$ experiments at high spinning frequencies and long contact times for model compounds with long $\mathrm{T}_{1}{ }_{\rho}{ }^{\mathrm{H}}$ relaxation times. Since ramped $\mathrm{CP}$ is applied at high spinning frequencies, the spinning sidebands are significantly reduced and thus, in some cases, the resulting spectrum resembles the DP/MAS spectrum. However, it should be noted that the polarization transfer in ramped CP is slower than that in regular $\mathrm{CP}$ at lower spinning frequencies, because for a given site in the coil the matching condition is fulfilled only during part of the contact time. It has been demonstrated that a contact time of $10 \mathrm{~ms}$ is needed to obtain quantitative spectra $[58,59]$, which is usually not a problem for model compounds. However, for NOM samples such as humic acids (HAs) with short $\mathrm{T}_{1 p}{ }^{\mathrm{H}}$ relaxation times of only a few milliseconds, significant and often differential signal losses occur during long, ramped CP periods. As shown in Fig. 7, a contact time as short as $2 \mathrm{~ms}$ leads to significant and differential relaxation; after $5 \mathrm{~ms}$, most signals have decayed; and at $10 \mathrm{~ms}$, almost none remain. This clearly indicates that ramp-CP does not give quantitative spectra of NOM.

This problem is mostly overcome by the recently developed multiple CP (multiCP) technique [27]. Using this technique, quantitative ${ }^{13} \mathrm{C}$ NMR spectra of organic materials with good signal-to-noise ratios can be obtained in a relatively short time. More than $10 \mathrm{~ms}$ long CP 
from ${ }^{1} \mathrm{H}$ can be implemented without significant magnetization losses due to relaxation by using multiple 1-ms ramped-CP periods alternating with ${ }^{1} \mathrm{H}$ spin-lattice relaxation periods that repolarize the protons. The acquired signal intensity is quite insensitive to the exact field strengths used, due to the ramp of the radio-frequency field strength and the asymptotic limit of cross polarization. At a spinning frequency of $14 \mathrm{kHz}$, spinning sidebands are fairly small $(\leq$ $10 \%$ ) and have little overlap with centerbands. Corresponding multiCP spectra of nonprotonated $\mathrm{C}$ and mobile groups like rotating $\mathrm{CH}_{3}$ can also be obtained by combining the multiCP sequence with 68- $\mu$ s recoupled dipolar dephasing [63]. Due to a twice stronger signal and a $\sim 15$-fold shorter time per scan, multiCP typically reduces the measuring time by more than a factor of 50 compared to quantitative ${ }^{13} \mathrm{C}$ DP/MAS [27]. Thus it enables higherthroughput quantitative NMR studies, in particular for relatively 'clean' organic materials with relatively long relaxation times. However, since this technique is based on $\mathrm{CP}$, it will not give quantitative ${ }^{13} \mathrm{C}$ spectra for samples lacking protons such as diamond or chars made by hightemperature pyrolysis.

\subsection{Peak overlap}

As shown in Fig. 1, most ${ }^{13} \mathrm{C}$ spectra of NOM are broad and the assignment of specific functional groups is difficult due to heavy band overlap. One approach that has been used for extracting structural information from these heavily overlapped spectra is integration [1, 30]. Usually, a broad NOM spectrum such as the one in Fig. 1 is arbitrarily separated into 5-10 different regions, and then each region is assigned to a group of functional groups. However, this integration approach is rather inaccurate. For example, the $90-120$ ppm range in a typical NOM spectrum contains signal contributions from both $\mathrm{sp}^{3}$-hybridized anomeric carbons and 
$\mathrm{sp}^{2}$-hybridized aromatic carbons. Thus, the integration region assigned to aromatics inevitably contains some aliphatics and vice versa. Another approach to dealing with complex NOM spectra is deconvolution [48, 64]. In this approach, different peaks are fitted into the broad NOM bands so that the sum of the fitting peaks matches the experimental spectrum. Deconvolution is useful and reliable if it is known which components are present and what their peak positions and widths are. However, if the identities of specific functional groups and their peak positions and widths within a broad spectral band are not accurately known, as is usually the case for NOM spectra, then the fitting is ambiguous since different combinations of peaks can fit the same band. A better approach for identifying the signals of specific functional groups in heavily overlapped ${ }^{13} \mathrm{C}$ NOM spectra is to use the spectral-editing techniques detailed in Section 3.

\subsection{Observability and spin counting}

It has been widely recognized that some NOM carbons are potentially not visible in NMR experiments. Various factors contribute to this signal loss. First of all, NOM is one of the most complex, heterogeneous carbonaceous materials known. This means that nuclei of different functional groups would be expected to have quite disparate spin dynamics. For example, the

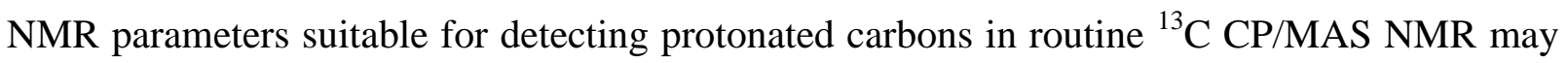
cause low detectability of fused-ring aromatic carbons. Second, almost all NOM samples contain some inorganic materials, many of which include paramagnetic ions. The presence of paramagnetic materials can significantly broaden carbon signals, rendering these signals difficult to detect fully. Short ${ }^{1} \mathrm{H} T_{1}$ relaxation times on the order of $20 \mathrm{~ms}$ indicate that some NOM samples also contain organic free radicals, which can similarly suppress signals of 
nearby carbons. In addition, as mentioned above, poor cross polarization or loss of the initial signal during the pulse dead time leads to poor quality spectra and partial loss of some NMR signals.

In the very first use of ${ }^{13} \mathrm{C}$ CP NMR for the study of coal, VanderHart and Retcofsky [65] estimated that significant amounts of the total carbon spins present in the sample were not detected. Since then, the issue of detectability in NMR analyses of NOM has been a topic of much debate (see references in [66]). The general consensus among the NOM community is that a substantial fraction of carbon atoms is not observed in CP spectra [51].

Spin counting can be used to estimate the proportion of the total NMR signal detected in a measured spectrum. In this approach, the total signal intensity of an NOM sample with known mass and carbon content is compared with that of a model compound which gives a quantitative signal. Spin counting has been extensively used for coal studies [66]. Depending on the rank and maceral types, the CP NMR observability of carbons in coals varies [66]. A few studies have also performed spin counting on soil organic matter. Preston and Newman [67] found that carbon detectability was in the range of $10-30 \%$ for soil particle size fractions, and Preston et al. [68] reported carbon detectability between 7.6-37\% for HF-treated humin fractions. Smernik and Oades [51] investigated the carbon detectability of HF-treated, $<53 \mu \mathrm{m}$ fractions of eight soils and found that carbon observability in DP/MAS experiments was between 84-106\%, whereas that in CP/MAS was between 54-83\%. Keeler and Maciel [69] performed spin counting experiments on a whole forest soil with a fairly high carbon content, low iron content, and short ${ }^{13} \mathrm{C} \mathrm{T}_{1}$, the HF-treated whole soil, and its extracted humin, humic acid, and fulvic acid fractions using both CP and DP. They reported 59-72\% detectability of the carbons for ${ }^{13} \mathrm{C} \mathrm{DP}$ and $48.4 \%-68.5 \%$ for ${ }^{13} \mathrm{C} \mathrm{CP}$; they also showed that $\mathrm{HF}$ increased 
carbon detectability for both DP and CP experiments. Fang et al. [70] conducted spin counting experiments on Iowa Mollisols. DP/MAS detected $\sim 68 \%$ of organic $\mathrm{C}$ in untreated soil, and close to $100 \%$ after HF treatment to concentrate the organic matter. This was probably the first study presenting reliable, quantitative DP NMR spectra of untreated whole soils with $\leq 3$ wt\% C, where most (approximately $68 \%$ ) of organic $\mathrm{C}$ was observed.

In summary, variable proportions of NOM carbon signals may not be detectable in NMR experiments, depending on the sample characteristics and NMR setup; ${ }^{13} \mathrm{C}$ DP leads to higher carbon detectability than does ${ }^{13} \mathrm{C} \mathrm{CP}$, and HF treatment enhances carbon detectability.

\section{Identification of specific functional groups}

\subsection{Introduction to spectral-editing techniques}

Spectral-editing techniques greatly assist peak assignment and simplify complex spectra [71-75]. In solution ${ }^{13} \mathrm{C}$ NMR, various spectral-editing techniques such as distortionless enhancement by polarization transfer (DEPT) and the attached-proton test (APT) are used to distinguish $\mathrm{CH}_{3}, \mathrm{CH}_{2}$, and $\mathrm{CH}$ groups In the solid state, these $\mathrm{J}$-coupling based spectral-editing techniques are only applicable to crystalline materials with long transverse relaxation times [76-78].

Nevertheless, spectral editing is also of interest to NMR spectroscopists who study NOM, since it reduces peak overlap, helps with peak assignment, and provides new information, such as the fraction of nonprotonated carbons. Solid-state spectral-editing techniques suitable for NOM can be based on differences in (1) dipolar couplings (which include CP dynamics), (2) chemical shift anisotropies, (3) evolution of multi-spin states, and (4) relaxation times. The most commonly used technique is routine dipolar dephasing at spinning frequencies $<10 \mathrm{kHz}$ 
[73]. In this technique, ${ }^{1} \mathrm{H}$ decoupling is gated off for a brief period, usually $40 \mu$ s. During this time, magnetization from strongly proton-coupled carbons decays, while the resonances from weakly proton-coupled carbons such as quaternary carbons and mobile segments are retained. Thus, the dipolar dephasing technique selects signals from nonprotonated carbons and mobile groups, including rotating methyl groups.

Under fast MAS (>12 kHz), gating the ${ }^{1} \mathrm{H}$ decoupling off for a half or even a full rotation period does not suppress the protonated-carbon signals, since MAS reverses the effects of the C-H dipolar couplings. This problem can be overcome by simple recoupling: If the period without ${ }^{1} \mathrm{H}$ irradiation is centered on the $\pi$-pulse in the Hahn echo before detection, the pulse prevents MAS from reversing the $\mathrm{C}-\mathrm{H}$ dephasing, and suppression of $\mathrm{C}-\mathrm{H}$ signals can be achieved in a total gated-decoupling time of $\sim 68 \mu$ s [47].

To identify immobile methylene groups, cross polarization under windowless isotropic mixing for spectral editing (WIMSE) [79] was developed: Under homonuclear decoupling of protons, cross polarization of $\mathrm{CH}$ spin pairs is reversed by MAS, while the interference between the two $\mathrm{C}-\mathrm{H}$ dipolar couplings in an immobile methylene group prevents a reversal of $\mathrm{CP}$; so ideally only $\mathrm{CH}_{2}$ signals remain after full rotation periods. However, due to long-range $\mathrm{C}-\mathrm{H}$ couplings and imperfect homonuclear decoupling, the selection is not clean and puremethylene spectra were obtained only as the weighted difference between two spectra, with an empirical scaling factor [79]. A series of spectral techniques which can select $\mathrm{CH}, \mathrm{CH}_{2}, \mathrm{CH}_{3}$ and quaternary carbons was developed [80-84] based on carefully chosen CP periods, cross depolarization, and polarization inversion. Clean selection was only accomplished for immobile $\mathrm{CH}_{2}$ and quaternary carbons at standard MAS rates [80-83]. Selection of $\mathrm{CH}$ and $\mathrm{CH}_{3}$ groups was achieved only partially, and therefore pure $\mathrm{CH}$ - and $\mathrm{CH}_{3}$ - spectra needed to be 
generated as linear combinations of various spectra using coefficients empirically determined from model compounds. The $\mathrm{CH}_{2}$ selection depends strongly on the $\mathrm{CP}$ conditions and is sensitive to spectrometer drift [85]. In addition, these techniques do not work well when C-H dipolar couplings are reduced by segmental motions. Later, Hu et al. modified these spectralediting techniques [86], and the resulting techniques have been applied to a humic sample [87], dried pine leaves [88], and a soil sample treated with $2 \% \mathrm{HF}$ [89]. However, spectral quality is not sufficient for complex organic matter, as partially demonstrated in [85]. Saalwächter et al. [90] and De Vita \& Frydman [91] selectively suppressed $\mathrm{CH}_{2}$ signals based on solid-state dipolar insensitive nuclei enhanced by polarization transfer (INEPT) at high spinning frequencies. Although this approach can cleanly suppress $\mathrm{CH}_{2}$ peaks in rigid systems, the absolute $\mathrm{CH}$ signal intensities are low because, to reach high spinning frequencies, small rotors and thus small amounts of samples must be used. Further, mobile $\mathrm{CH}_{2}$ groups are often not suppressed; this has been taken advantage of for the characterization of $\mathrm{CH}_{2}$ mobility [92]. Recently, a method for separating NMR resonances from protonated and non-protonated aromatic carbons in solids under fast MAS was proposed by employing a spin-echo without ${ }^{1} \mathrm{H}$ decoupling for several milliseconds [93]. Peng and Frydman [94] employed differences in the ${ }^{13} \mathrm{C}$ chemical-shift anisotropies (CSAs) to distinguish $\mathrm{sp}^{3}$-hybridized carbons, with small CSAs due to their nearly tetrahedral bonding symmetry, from $\mathrm{sp}^{2}$-hybridized carbons, which have larger CSAs. However, due to limitations of the pulse sequence, the selection was not very clean, with dispersive spinning sidebands remaining.

Carbons in different domains can be distinguished based on differences in the $T_{1 \rho}, T_{1}$ and $\mathrm{T}_{2}$ relaxation times of nearby protons in CP/MAS NMR [95-101]. Newman introduced the proton spin relaxation editing (PSRE) approach [102] to obtain the subspectra of different 
domains in heterogeneous organic matter. The PSRE technique, which is essentially a modified version of the ${ }^{1} \mathrm{H}$ inversion recovery technique, has been used extensively to study organic matter [103-107]. Straightforward ${ }^{1} \mathrm{H}$ inversion recovery is useful to selectively observe the signal of crystalline polymethylene domains near $33 \mathrm{ppm}$, which are free of unpaired electrons and therefore have particularly long ${ }^{1} \mathrm{H} \mathrm{T}_{1}[108,109] .{ }^{13} \mathrm{C}$ relaxation time differences are also used to differentiate carbons with different segmental mobilities or proximities to unpaired electrons. Specifically, ${ }^{13} \mathrm{C}_{1}$ and $\mathrm{T}_{2}$ filters can be used for discriminating carbon components with different ${ }^{13} \mathrm{C}_{1}$ and $\mathrm{T}_{2}$ values $[24,56,110,111]$. For example, in order to highlight mobile functional groups, ${ }^{13} \mathrm{C}$ DP/MAS spectra with a short recycle delay of $1.5 \mathrm{~s}$ can be used.

Nevertheless, apart from slow-MAS dipolar dephasing and relaxation-time based techniques, spectral editing had barely been applied to study NOM before the authors started to develop robust spectral-editing techniques suitable for NOM, based on multi-spin dynamics or CSA differences. As described in following sections, these techniques include selection of $\mathrm{sp}^{3}$ hybridized carbon signals by a ${ }^{13} \mathrm{C}$ CSA filter [112], $\mathrm{CH}$ selection by dipolar DEPT [113], $\mathrm{CH}_{2}$ selection by three-spin coherence selection [85], selection of nonprotonated C under fast MAS by recoupled dipolar dephasing [47], selection of signals of interior carbons in fused ring carbons by recoupled long-range dipolar dephasing [114], selection of signals of carbons bonded to nitrogen by saturation-pulse induced dipolar exchange with recoupling (SPIDER) [115], and selection of nonprotonated nitrogen by ${ }^{15} \mathrm{~N}$ CP/MAS with recoupled dipolar dephasing [116]. In addition, signals of protonated carbons can be selected by recording a CP spectrum with a short contact time of $50 \mu$ s, running the same experiment with dipolar dephasing before detection, and taking the difference between the two spectra. The second spectrum contains only the signals of the nonprotonated carbons, which are therefore removed 
when the difference is taken. Figure 8 demonstrates the application of these advanced spectralediting techniques to a complex fulvic acid from Pony Lake in Antarctica.

\subsection{Principles and practice of some recently developed spectral-editing techniques}

\subsection{1. ${ }^{13}$ C chemical shift anisotropy filter}

\subsubsection{Introduction}

For ${ }^{13} \mathrm{C}$ spectra of complex NOM, the signals of $\mathrm{sp}^{2}$-hybridized aromatics and $\mathrm{sp}^{3}$ hybridized aliphatics overlap around 90-120 ppm. To separate them, the difference in CSA between $\mathrm{sp}^{2}$-hybridized and $\mathrm{sp}^{3}$-hybridized carbons is explored to generate a subspectrum containing only $\mathrm{sp}^{3}$-hybridized carbon signals by suppressing $\mathrm{sp}^{2}$-hybridized carbon signals using a ${ }^{13} \mathrm{C}$ CSA filter [112]. This technique relies on the differences in carbon bonding symmetry, as reflected in the respective sizes of the ${ }^{13} \mathrm{C}$ CSA. In a perfectly tetrahedral environment, the CSA disappears [117]. Therefore, the ${ }^{13} \mathrm{C}$ CSA values of $\mathrm{sp}^{3}$-hybridized carbons with nearly tetrahedral bonding are much smaller than those of $\mathrm{sp}^{2}$-hybridized carbons with planar bonding [117]. When the chemical shift anisotropy is recoupled using a few rotation-synchronized $\pi$-pulses, the magnetization of $\mathrm{sp}^{2}$-hybridized carbons with large CSAs decays faster than that of $\mathrm{sp}^{3}$-hybridized carbons with small CSAs. Therefore, after an appropriate recoupling time, the magnetization of $\mathrm{sp}^{2}$-hybridized carbons is dephased whereas that of $\mathrm{sp}^{3}$-hybridized carbons is retained and subsequently detected.

\subsubsection{Theoretical background}

There are two different pulse sequences used in the authors' laboratories for the ${ }^{13} \mathrm{C}$ CSA filter technique, referred to as three- and five-pulse versions (counting both the $\pi$ and the $\pi / 2$ 
pulses). Only the theoretical background of three-pulse version is described here, but that of the five-pulse version of ${ }^{13} \mathrm{C}$ CSA dephasing can be derived similarly.

The simple, three-pulse version adopts a single $\pi$ pulse in the middle of a period $2 t_{\text {csa }} \leq t_{r}$, which is similar to that proposed by Peng and Frydman [94]. However, the sequence has been improved by applying a $z$-filter incremented in 4 steps of $t_{r} / 4$ before detection. This " $\gamma$ averaging" removes broad dispersive wings of sidebands and is required for TOSS to properly suppress sidebands up to $4^{\text {th }}$ order [118]. The $\pi$-pulse plus the two $\pi / 2$ pulses for $z$-storage and read-out are the three pulses mentioned above.

For the detected signal $s_{\text {deph }}(t)$, the signal of a given carbon site at $t=0$ is

$$
\mathrm{s}_{\mathrm{deph}}(0)=\mathrm{s}(0)\left\langle\cos \left[\Phi_{\mathrm{tot}}\left(2 \mathrm{t}_{\mathrm{csa}}\right)\right]\right\rangle
$$

where the angle brackets refer to the powder average; $s(0)$ indicates the signal intensity without a CSA filter; and $\mathrm{s}_{\mathrm{deph}}(0)$ represents the overall signal of the carbon site in the CSA-filtered spectrum. The argument of the cosine dephasing function is the total phase of the magnetization due to CSA evolution during the two $\mathrm{t}_{\mathrm{csa}}$ periods:

$$
\begin{aligned}
& \Phi_{\text {tot }}\left(2 t_{C S A}\right)=-\int_{0}^{t_{C S A}} \omega(t) d t+\int_{t_{C S A}}^{2 t_{C S A}} \omega(t) d t \\
& =-\int_{0}^{t_{C S A}} \omega(t) d t+\int_{0}^{2 t_{C S A}} \omega(t) d t-\int_{0}^{t_{C S A}} \omega(t) d t \\
& =\Phi\left(2 t_{\mathrm{csa}}\right)-2 \Phi\left(t_{\mathrm{csa}}\right) \propto \delta_{\mathrm{CSA}}
\end{aligned}
$$


is the phase acquired by the magnetization during a time $t$ without pulses under the influence of the CSA during magic angle spinning. The time dependence of the phase in eq.(6) has been described elsewhere [26]. Most importantly, the dephasing increases proportionally to the CSA parameter $\delta_{\text {CSA }}$. These parameters are dependent on the segmental orientations usually expressed by the Euler angles $(\alpha, \beta, \gamma)$ relative to a rotor-fixed frame. Incrementation of the $z$ period over one rotation period achieves the " $\gamma$-averaging". The signal intensity can then be fully parameterized in terms of $\delta_{\mathrm{CSA}}, \mathrm{t}_{\mathrm{CSA}}, \omega_{\mathrm{r}}$, and $\eta$. After $2 \mathrm{t}_{\mathrm{CSA}}=2 \times 70 \mu \mathrm{s}$ the signals of aromatic carbons can all be dephased [26].

In order to select the signals of nonprotonated O-C-O (ketal) carbons, which may extend up to $120 \mathrm{ppm}$, the CSA filter is combined with a dipolar dephasing time of $40 \mu \mathrm{s}$. In a complementary experiment, selective spectra of protonated anomerics (O-CH-O, acetals) can be achieved by the combination of the CSA filter with short CP of $50 \mu$ s [112].

\subsubsection{Practical considerations}

Using pulse lengths and other parameters from the optimized ${ }^{13} \mathrm{C} C P / T O S S$ experiment, the

${ }^{13} \mathrm{C}$ CSA filter experiment can be easily set up by selecting an appropriate CSA filter time and choosing the increment (typically $t_{\mathrm{r}} / 4$ ) of the $z$-period for " $\gamma$-averaging". The ${ }^{13} \mathrm{C}$ CSA filter time should be chosen according to the $\mathrm{B}_{0}$ magnetic field strength. The spinning frequency can range from 5 to $6 \mathrm{kHz}$ at $9.4 \mathrm{~T}$, and proportionally faster at higher fields. The five-pulse version extends the dephasing to up to two rotation periods and is therefore applicable at up to twice faster spinning frequencies (i.e. $12 \mathrm{kHz}$ at $9.4 \mathrm{~T}$ ).

\subsection{2. $\mathrm{CH}$ selection}




\subsubsection{Theoretical background}

The dipolar-DEPT CH selection technique [113], which is analogous to DEPT in solution NMR [75, 119], is based on the invariance of $\mathrm{C}-\mathrm{H}$ multiple-quantum (MQ) coherence under the $\mathrm{C}-\mathrm{H}$ dipolar coupling as opposed to the efficient dephasing of the corresponding coherence in a $\mathrm{CH}_{2}$ group by the second proton. In order to reduce quaternary-carbon and $\mathrm{CH}_{3}$ signals and to minimize the duration of the pulse sequence, $\mathrm{C}-\mathrm{H}$ multiple-quantum excitation and reconversion for time periods much shorter than a rotation period are implemented. Without relying on specific coupling strengths, this technique can even be applied in the presence of partial motional averaging and suppress mobile- $\mathrm{CH}_{2}$ and quaternary-carbon signals, efficiently retaining $\mathrm{CH}$ peaks up to $14 \%$. The heteronuclear $\mathrm{C}-\mathrm{H}$ coherence calculation is demonstrated in the following.

For the spin-pair dipolar coupling $\mathrm{H}_{\mathrm{IS}}=\omega_{\mathrm{CH}} \mathrm{S}_{\mathrm{Z}} \mathrm{I}_{\mathrm{Z}}$ of a $\mathrm{CH}$ group, heteronuclear multiplequantum coherence $S_{x / y} I_{x / y}$ of an S-I spin-pair such as $\mathrm{CH}$ does not change because $\left[\mathrm{H}_{\mathrm{IS}}\right.$, $\left.S_{x / y} I_{x / y}\right]=0$. In contrast, for a $\mathrm{CH}_{2}$ group with its two protons $A$ and $B, S_{x / y} I_{x / y A}$ does not commute with the second term of $\mathrm{H}_{\mathrm{IS}}=\omega_{\mathrm{CH}, \mathrm{A}} \mathrm{S}_{\mathrm{Z}} \mathrm{I}_{\mathrm{ZA}}+\omega_{\mathrm{CH}, \mathrm{B}} \mathrm{S}_{\mathrm{Z}} \mathrm{I}_{\mathrm{ZB}}$ and therefore is rapidly dephased. The product-operator calculations of the density operator in terms of excitation, MQ evolution, reconversion, and identification of the observable terms at the start of detection for $\mathrm{CH}, \mathrm{CH}_{2}$, and $\mathrm{CH}_{3}$ are as follows [113], with sine and cosine modulations due to heteronuclear

dipolar evolution abbreviated as s and c, respectively, and the powder-averaged signal intensity shown after ' $\rightarrow$ ':

$\mathrm{CH}$ :

Excitation: $\rho\left(\mathrm{t}_{\mathrm{CH}}\right)=\mathrm{I}_{\mathrm{x}} \mathrm{c}+\mathbf{2} \mathbf{S}_{\mathbf{z}} \mathbf{I}_{\mathbf{y}} \mathbf{s}$ 
MQ evolution: $\rho\left(\mathrm{t}_{\mathrm{CH}}+\mathrm{t}_{\mathrm{MQ}}\right)=\mathbf{2} \mathbf{S}_{\mathbf{y}} \mathbf{I}_{\mathbf{y}} \mathbf{S}$ unchanged

Reconversion: $\rho\left(2 \mathrm{t}_{\mathrm{CH}}+\mathrm{t}_{\mathrm{MQ}}\right)=2 \mathrm{~S}_{\mathrm{y}} \mathrm{I}_{\mathrm{z}} \mathrm{s} \mathrm{c}-\mathbf{S}_{\mathrm{x}} \mathbf{s}^{2}$

Observable: $\quad \rho_{\text {obs }}\left(2 \mathrm{t}_{\mathrm{CH}}+\mathrm{t}_{\mathrm{MQ}}\right)=-\mathrm{S}_{\mathrm{x}} \mathrm{s}^{2} \rightarrow 0.5$

$\mathrm{CH}_{2}$ :

Excitation: $\rho\left(t_{C H}\right)=I_{x A} c_{A}+I_{x B} c_{B}+2 S_{\mathbf{z}}\left(\mathbf{I}_{\mathbf{y A}} \mathbf{S}_{\mathbf{A}}+\mathbf{I}_{\mathbf{y B}} \mathbf{S}_{\mathbf{B}}\right)$

MQ evolution: $\left.\rho\left(\mathrm{t}_{\mathrm{CH}}+\mathrm{t}_{\mathrm{MQ}}\right)=\mathbf{2} \mathbf{I}_{\mathbf{y A}} \mathbf{s}_{\mathbf{A}}\left(\mathbf{S}_{\mathbf{y}} \mathbf{c}_{\mathbf{B}}^{\prime}-2 \mathrm{I}_{\mathrm{zB}} \mathrm{S}_{\mathrm{x}} \mathrm{s}_{\mathrm{B}}^{\prime}\right)\right)$

$$
+2 I_{\mathbf{y B}} \mathbf{S}_{\mathbf{B}}\left(\mathbf{S}_{\mathbf{y}} \mathbf{c}^{\prime}{ }_{\mathbf{A}}-2 \mathrm{I}_{\mathrm{zA}} \mathrm{S}_{\mathrm{x}} \mathrm{s}_{\mathrm{A}}^{\prime}\right)
$$

Reconversion: $\rho\left(2 \mathrm{t}_{\mathrm{CH}}+\mathrm{t}_{\mathrm{MQ}}\right)=2\left[\mathbf{I}_{\mathbf{z A}} \mathbf{s}_{\mathbf{A}} \mathbf{c}_{\mathbf{B}}^{\prime}+\mathbf{I}_{\mathbf{z B}} \mathbf{S}_{\mathbf{B}} \mathbf{c}_{\mathrm{A}}^{\prime}\right]$

$$
\begin{aligned}
& \left(\mathrm{S}_{\mathrm{y}}\left(\mathrm{c}_{\mathrm{A}} \mathrm{c}_{\mathrm{B}}-4 \mathrm{I}_{\mathrm{zA}} \mathrm{I}_{\mathrm{zB}} \mathrm{S}_{\mathrm{A}} \mathrm{S}_{\mathrm{B}}\right)\right. \\
& \left.-\mathbf{2 S}_{\mathbf{x}}\left(\mathbf{I}_{\mathbf{z A}} \mathbf{s}_{\mathbf{A}} \mathbf{c}_{\mathbf{B}}+\mathbf{I}_{\mathbf{z B}} \mathbf{c}_{\mathbf{A}} \mathbf{S}_{\mathbf{B}}\right)\right)
\end{aligned}
$$

Observable: $\rho_{\mathrm{obs}}\left(2 \mathrm{t}_{\mathrm{CH}}+\mathrm{t}_{\mathrm{MQ}}\right)=-\mathrm{S}_{\mathrm{x}}\left(\mathrm{s}_{\mathrm{A}}^{2} \mathrm{c}_{\mathrm{B}} \mathrm{c}_{\mathrm{B}}^{\prime}+\mathrm{s}_{\mathrm{B}}^{2} \mathrm{c}_{\mathrm{A}} \mathrm{c}_{\mathrm{A}}^{\prime}\right) \rightarrow \mathbf{0}$

\section{$\mathrm{CH}_{3}$ :}

Excitation: $\rho\left(\mathrm{t}_{\mathrm{CH}}\right)=\left(\mathrm{I}_{\mathrm{xA}}+\mathrm{I}_{\mathrm{xB}}+\mathrm{I}_{\mathrm{xC}}\right) \mathrm{c}+\mathbf{2} \mathbf{S}_{\mathbf{z}}\left(\mathbf{I}_{\mathbf{y A}}+\mathbf{I}_{\mathbf{y B}}+\mathbf{I}_{\mathbf{y C}}\right) \mathbf{s}$

MQ evolution: $\rho\left(t_{\mathrm{CH}}+\mathrm{t}_{\mathrm{MQ}}\right)=\mathbf{2 s}\left\{\mathbf{S}_{\mathbf{y}}\left[\mathbf{c}^{\mathbf{\prime}^{2}}\left(\mathbf{I}_{\mathbf{y A}}+\mathbf{I}_{\mathbf{y B}}+\mathbf{I}_{\mathbf{y C}}\right)\right.\right.$

$$
\begin{aligned}
& \left.-4 \mathrm{~s}^{\prime 2}\left(\mathrm{I}_{\mathrm{yA}} \mathrm{I}_{\mathrm{zB}} \mathrm{I}_{\mathrm{zC}}+\mathrm{I}_{\mathrm{yB}} \mathrm{I}_{\mathrm{zC}} \mathrm{I}_{\mathrm{zA}}+\mathrm{I}_{\mathrm{yC}} \mathrm{I}_{\mathrm{zA}} \mathrm{I}_{\mathrm{zB}}\right)\right] \\
& -\mathrm{S}_{\mathrm{x}} 2 \mathrm{c}^{\prime} \mathrm{s}^{\prime}\left[\mathrm{I}_{\mathrm{yA}}\left(\mathrm{I}_{\mathrm{zB}}+\mathrm{I}_{\mathrm{zC}}\right)+\mathrm{I}_{\mathrm{yB}}\left(\mathrm{I}_{\mathrm{zC}}+\mathrm{I}_{\mathrm{zA}}\right)+\mathrm{I}_{\mathrm{yC}}\left(\mathrm{I}_{\mathrm{zA}}+\mathrm{I}_{\mathrm{zB}}\right)\right\}
\end{aligned}
$$

Reconversion: $\rho\left(2 t_{C H}+t_{M Q}\right)=\mathbf{2}\left(\mathbf{I}_{\mathbf{z A}}+\mathbf{I}_{\mathbf{z B}}+\mathbf{I}_{\mathbf{z C}}\right) \mathbf{s ~ c}^{\mathbf{\prime}^{2}}$

$$
\begin{aligned}
& \left\{S_{y}\left[c^{3}-4\left(I_{z A} I_{z B}+I_{z A} I_{z C}+I_{z B} I_{z C}\right) s^{2} c\right]\right. \\
& \left.\quad-S_{\mathbf{x}}\left[\mathbf{c}^{2} \mathbf{s} \mathbf{2}\left(I_{\mathbf{z A}}+\mathbf{I}_{\mathbf{z B}}+\mathbf{I}_{\mathbf{z C}}\right)-8 \mathrm{I}_{\mathrm{zA}} I_{z B} I_{z C} \mathbf{s}^{3}\right]\right\}
\end{aligned}
$$

Observable: $\rho_{\text {obs }}\left(2 t_{C H}+t_{M Q}\right)=-S_{x} 3 s^{2} c^{2} c^{\prime 2} \rightarrow 3 / 16=0.1875$ 
For a clean selection of $\mathrm{CH}$ signals, the $\mathrm{CH}_{2}$ suppression described so far is combined with suppression of quaternary-C signals via inverse dipolar dephasing, and reduction of $\mathrm{CH}_{3}$ signals via $T_{1 \mathrm{C}}$ relaxation [113].

\subsubsection{Practical considerations}

The initial setup of the experiment for $\mathrm{CH}$ selection can be challenging. Use of a model compound such as cholesteryl acetate for setting up the experiment is recommended. For homonuclear decoupling, it involves the use of the semi-windowless Mansfield-RhimElleman-Vaughn (MREV) pulse sequence [120], with ${ }^{1} \mathrm{H}$ pulse flip angles close to $109^{\circ}$. For fairly rigid solids, convenient pulse timings and a good signal-to-noise ratio are achieved at a spinning frequency near $5.8 \mathrm{kHz}$. By running the experiment at the same spinning frequency for all samples, re-optimization of pulse timings is avoided and only a few power levels need to be adjusted (to produce the required flip angles) when the method is applied to a new sample. If intense signals of highly mobile $\mathrm{CH}_{2}$ groups present a problem, a lower spinning frequency of $4 \mathrm{kHz}$ is recommended, since the resulting longer rotation period provides more time for dephasing of the multiple-quantum coherence of the mobile $\mathrm{CH}_{2}$ groups by the relatively weak second $\mathrm{C}-\mathrm{H}$ coupling.

\subsection{3. $\mathrm{CH}_{2}$ selection}

\subsubsection{Theoretical background}

CP-based $\mathrm{CH}_{2}$-selection techniques are highly sensitive to accurate setting of the Hartmann-Hahn match conditions and therefore require careful adjustment [80, 86, 87]. A more robust $\mathrm{CH}_{2}$ selection technique has overcome this limitation as it does not rely on a steep 
zero-crossing or sensitive Hartmann-Hahn match condition [85]. This technique is based on the selection of the characteristic three-spin coherence of $\mathrm{CH}_{2}$ groups, using a ${ }^{13} \mathrm{C} 90^{\circ}$ pulse and ${ }^{1} \mathrm{H}$ $0^{\circ} / 180^{\circ}$ pulses applied after the first quarter of one rotation period with MREV-8 decoupling. First, ${ }^{13} \mathrm{C}$ magnetization of $\mathrm{CH}_{2}$ groups is converted into two- and three-spin coherences during 0.043-ms of heteronuclear dipolar evolution under MREV-8 homonuclear proton decoupling. A ${ }^{1} \mathrm{H} 0^{\circ} / 90^{\circ}$ pulse composed of two $45^{\circ}$ pulses and the removal of two-spin coherence $\mathrm{S}_{\mathrm{y}} \mathrm{I}_{\mathrm{z}}$ by a ${ }^{13} \mathrm{C} 90^{\circ} \mathrm{x}$-pulse (see details below) allows the three-spin coherence $\mathrm{S}_{\mathrm{x}} \mathrm{I}_{\mathrm{Z}} \mathrm{J}_{\mathrm{Z}}$ in the $\mathrm{S} \mathrm{I} \mathrm{J}(\mathrm{C} \mathrm{H} \mathrm{H})$ spin system to be distinguished from ${ }^{13} \mathrm{C}$ magnetization $\left(S_{\mathrm{x}}\right)$. Finally, the magnetization for detection is produced by reconverting the three-spin coherence.

For a $\mathrm{CH}$ group of an $\mathrm{S}$ and an $\mathrm{I}$ spin, with carbon magnetization $\mathrm{S}_{\mathrm{x}}$ evolving under the heteronuclear dipolar coupling of strength $\omega_{\text {IS }}(t)$ [85], the density operator after a time $\tau$ of $t_{r} / 4$ with this $\mathrm{CH}_{2}$ technique is given by [26]

$$
\rho(\tau)=S_{\mathrm{x}} \cos \Phi_{\mathrm{IS}}+2 \mathrm{~S}_{\mathrm{y}} \mathrm{I}_{\mathrm{z}} \sin \Phi_{\mathrm{IS}}
$$

The time evolution of $\mathrm{S}_{\mathrm{x}}$ in a $\mathrm{CH}_{2}$ group with protons $\mathrm{I}$ and $\mathrm{J}$ produces three-spin coherence:

$$
\begin{aligned}
\rho(\tau)= & \left(S_{\mathrm{x}} \cos \Phi_{\mathrm{JS}}+2 \mathrm{~S}_{\mathrm{y}} \mathrm{J}_{\mathrm{Z}} \sin \Phi_{\mathrm{JS}}\right) \cos \Phi_{\mathrm{IS}} \\
& +2\left(\mathrm{~S}_{\mathrm{y}} \cos \Phi_{\mathrm{JS}}-2 \mathrm{~S}_{\mathrm{x}} \mathrm{J}_{\mathrm{Z}} \sin \Phi_{\mathrm{JS}}\right) \mathrm{I}_{\mathrm{Z}} \sin \Phi_{\mathrm{IS}} \\
= & \mathrm{S}_{\mathrm{x}} \cos \Phi_{\mathrm{IS}} \cos \Phi_{\mathrm{JS}}+2 \mathrm{~S}_{\mathrm{y}} \mathrm{J}_{\mathrm{Z}} \sin \Phi_{\mathrm{JS}} \cos \Phi_{\mathrm{IS}} \\
& +2 \mathrm{~S}_{\mathrm{y}} \mathrm{I}_{\mathrm{Z}} \sin \Phi_{\mathrm{IS}} \cos \Phi_{\mathrm{JS}}-4 \mathrm{~S}_{\mathrm{X}} \mathrm{I}_{\mathrm{Z}} \mathrm{J}_{\mathrm{Z}} \sin \Phi_{\mathrm{IS}} \sin \Phi_{\mathrm{JS}}
\end{aligned}
$$


Since there is no three-spin coherence $\mathrm{S}_{\mathrm{X}} \mathrm{I}_{\mathrm{Z}} \mathrm{J}_{\mathrm{Z}}$ produced for a $\mathrm{CH}$ group, $\mathrm{CH}_{2}$ selection can be achieved by selecting the three-spin coherence and removing single- and two-spin coherences, and then converting the three-spin coherence back into observable ${ }^{13} \mathrm{C}$ magnetization. The two-spin terms $\mathrm{S}_{\mathrm{y}} \mathrm{I}_{\mathrm{z}}$ and $\mathrm{S}_{\mathrm{y}} \mathrm{J}_{\mathrm{z}}$ are effectively eliminated by a ${ }^{13} \mathrm{C} 90^{\circ} \mathrm{x}$ pulse at time $\tau$, which converts them to invisible, invariant $S_{Z} I_{Z}$ and $S_{z} J_{Z}$. The one-spin $\left(S_{x}\right)$ and threespin $\left(\mathrm{S}_{\mathrm{X}} \mathrm{I}_{\mathrm{Z}} \mathrm{J}_{\mathrm{Z}}\right)$ terms are distinguished by ${ }^{1} \mathrm{H}$ pulses. $\rho(\tau)$ is as follows after the ${ }^{13} \mathrm{C} 90^{\circ} \mathrm{x}$ pulse:

$$
\begin{aligned}
\rho(\tau)=S_{\mathrm{x}} & \cos \Phi_{\mathrm{IS}} \cos \Phi_{\mathrm{JS}}+2 \mathrm{~S}_{\mathrm{Z}} \mathrm{J}_{\mathrm{Z}} \sin \Phi_{\mathrm{IS}} \cos \Phi_{\mathrm{IS}} \\
& +2 \mathrm{~S}_{\mathrm{Z}} \mathrm{I}_{\mathrm{Z}} \cos \Phi_{\mathrm{IS}} \sin \Phi_{\mathrm{IS}}-4 \mathrm{~S}_{\mathrm{X}} \mathrm{I}_{\mathrm{Z}} \mathrm{J}_{\mathrm{Z}} \sin \Phi_{\mathrm{JS}} \sin \Phi_{\mathrm{IS}}
\end{aligned}
$$

The difference of scans with a $0^{\circ}$ and with a $90^{\circ}$ pulse on ${ }^{1} \mathrm{H}$ removes the signal from the single-spin magnetization $S_{x}$ (which is unaffected by the ${ }^{1} \mathrm{H}$ pulse) whereas signal from the three-spin coherence (which is dephased after a $90^{\circ}$ pulse on ${ }^{1} \mathrm{H}$ ) is retained. Their difference is as follows:

$$
\begin{aligned}
\rho_{0}(\tau)-\rho_{90}(\tau)= & 2\left(\mathrm{~S}_{\mathrm{z}} \mathrm{I}_{\mathrm{z}}-\mathrm{S}_{\mathrm{z}} \mathrm{I}_{\mathrm{y}}\right) \cos \Phi_{\mathrm{JS}} \sin \Phi_{\mathrm{IS}} \\
& +2\left(\mathrm{~S}_{\mathrm{z}} \mathrm{J}_{\mathrm{Z}}-\mathrm{S}_{\mathrm{z}} \mathrm{J}_{\mathrm{y}}\right) \sin \Phi_{\mathrm{JS}} \cos \Phi_{\mathrm{IS}} \\
- & 4\left(\underline{\mathrm{S}}_{\underline{\mathrm{I}}} \underline{\mathrm{I}}_{\underline{Z}} \underline{\mathrm{J}}_{\underline{z}}-\mathrm{S}_{\mathrm{x}} \mathrm{I}_{\mathrm{y}} \mathrm{J}_{\mathrm{y}}\right) \sin \Phi_{\mathrm{JS}} \sin \Phi_{\mathrm{IS}}
\end{aligned}
$$

Afterwards, the coherences further evolve under the heteronuclear dipolar couplings for a time $3 / 4 t_{r}$, and at the end of the rotation period only the original three-spin term is converted into observable $S_{\mathrm{x}}$ magnetization, $S_{\mathrm{x}} \sin ^{2} \Phi_{\mathrm{IS}} \sin ^{2} \Phi_{\mathrm{IS}}$. A short $100-\mu \mathrm{s}$ CP time at the start of the pulse sequence is used to suppress quaternary and methyl carbons, which have weaker $\mathrm{C}-\mathrm{H}$ couplings and therefore slower cross polarization than $\mathrm{CH}_{2}$ groups. A $z$-filter can be used on unlabeled samples to further suppress residual $\mathrm{CH}_{3}$ signals with short ${ }^{13} \mathrm{C}_{1}$ times due to 
methyl rotation. The period of heteronuclear evolution is kept short at $\tau=43 \mu$ s to avoid generating multi-spin coherence from long-range $\mathrm{C}-\mathrm{H}$ couplings.

\subsubsection{Practical considerations}

Similar to dipolar DEPT for $\mathrm{CH}$ selection, the $\mathrm{CH}_{2}$ selection also involves the setup of semi-windowless MREV-8, with a ${ }^{1} \mathrm{H}$ pulse flip angle of approximately $109^{\circ}$. Due to couplings of $\mathrm{CH}$ groups to more distant protons, $\mathrm{H}-\mathrm{C}-\mathrm{H}$ three-spin coherence is produced at a low level. The resulting signal can be canceled by increasing the flip angle of the ${ }^{13} \mathrm{C} x$-pulse at time $\tau$ to $\sim 110^{\circ}$, which generates a small compensating negative $\mathrm{CH}$ signal. The $\mathrm{CP}$ contact time should be set at $100 \mu \mathrm{s}$, and again cholesteryl acetate can be used as a model compound for setting up this technique.

\subsubsection{Recoupled ${ }^{1} \mathrm{H}^{-13} \mathrm{C}$ long-range dipolar dephasing}

\subsubsection{Introduction}

Long-range dipolar dephasing of ${ }^{13} \mathrm{C}$ by ${ }^{1} \mathrm{H}$ can be used to select the signals of interior carbons of fused-ring aromatics e.g. in chars. The simplest approach may be to increase the time without proton decoupling and to use cross polarization to generate the initial ${ }^{13} \mathrm{C}$ magnetization (for example, [121]). However, this pulse sequence has two shortcomings: (1) It relies on an inefficient and spinning-frequency-dependent second-order effect of dephasing, since to first order, magic-angle spinning reverses dipolar dephasing by the end of each full rotation period, and (2) loss of interior aromatic carbon signals due to the use of $\mathrm{CP}$. $\mathrm{CP}$ enhances the signals of carbons close to protons, but may fail to fully polarize carbons further away from protons such as those in fused-ring aromatics. In the ${ }^{1} \mathrm{H}-{ }^{13} \mathrm{C}$ recoupled dipolar 
dephasing technique [114], these problems are avoided. Here, refocusing by MAS is avoided by recoupling of the $\mathrm{C}-\mathrm{H}$ dipolar interactions, using two ${ }^{1} \mathrm{H} \pi$-pulses per rotation period, a $\mathrm{C}-\mathrm{H}$ version of the rotational-echo double-resonance (REDOR) [122] pulse sequence. A 0.9-ms recoupled dipolar dephasing period in a DP ${ }^{13} \mathrm{C}$ NMR experiment at $7 \mathrm{kHz}$ MAS can clearly distinguish between char and lignin in complex organic matter.

\subsubsection{Theoretical background}

(1) Reason for failure of long-time standard dipolar dephasing

In order to distinguish nonprotonated carbon sites at various distances from the nearest proton, longer dephasing times are necessary. Nevertheless, by simply increasing the dephasing time, the dephasing efficiency cannot be significantly increased. The reason is that MAS refocuses, at least partially, C-H dipolar couplings at the end of each rotation period, producing a rotational echo; this has been observed in various attempts to extend standard dipolar dephasing to longer times [121, 123, 124].

(2) Recoupling of long-range dipolar dephasing avoids refocusing by MAS

In this technique, the refocusing of anisotropic interactions by MAS is prevented by rotation-synchronized radio-frequency pulses. As shown by Gullion and Schafear [122], the recoupling of heteronuclear dipolar interactions can be achieved by using a REDOR train of $\pi$ pulses that are applied every half rotation period. For long-range ${ }^{13} \mathrm{C}\left\{{ }^{1} \mathrm{H}\right\}$ dephasing, the pulses are applied on ${ }^{1} \mathrm{H}$, except for one ${ }^{13} \mathrm{C} \pi$ pulse at the center of the recoupling period that refocuses ${ }^{13} \mathrm{C}$ chemical shift evolution. In addition, " $\gamma$-averaging" [118] by incrementing a zperiod before detection in $K$ steps of $t_{r} / K$ (typically with $K=4$ ) is employed to avoid phase distortions in MAS detection and sidebands in TOSS spectra [26]. The time constant of the 
dephasing reflects the strength of the $\mathrm{C}-\mathrm{H}$ couplings experienced by the ${ }^{13} \mathrm{C}$ sites. Protonproton homonuclear couplings do not significantly interfere with the C-H dephasing [125]. Finally, the effect of regular transverse $\left(\mathrm{T}_{2}\right)$ relaxation of ${ }^{13} \mathrm{C}$ is corrected for by recording the signal $\mathrm{S}_{0}$ using a reference sequence with a Hahn spin echo but with strong proton decoupling instead of the train of $\pi$-pulses.

\section{(3) Choice of direct polarization}

In this technique [114], direct polarization (DP) is employed instead of CP because longrange dipolar dephasing selects carbons far away from protons and CP distorts the spectra of fused-ring systems such as chars. DP can accurately represent most carbon signals of a fusedring system.

\subsubsection{Practical considerations}

At a given dephasing time, the ${ }^{1} \mathrm{H}$ recoupling $180^{\circ}$ pulses should be varied to minimize the residual signal intensity, which corresponds to optimum dephasing. Composite $\pi / 2_{x^{-}}-\pi_{y^{-}} \pi / 2_{x}$ ${ }^{1} \mathrm{H}$ pulses $[126,127]$ should be used in the pulse sequence to minimize the effects of flip-angle errors, e.g. due to $B_{1}$ inhomogeneity. The spinning frequency is typically $7 \mathrm{kHz}$. Faster spinning would result in a shorter rotation period and a larger fraction of the rotation period being taken up by the ${ }^{1} \mathrm{H}$ recoupling pulses; when that fraction exceeds $40 \%$, dephasing becomes compromised. Spectra are recorded for a series of recoupling times, which are even multiples of the rotation period, $N t_{\mathrm{r}}$. The reference signal, $S_{0}$, is usually recorded after $40-\mu \mathrm{s}$ dipolar dephasing of $\mathrm{C}-\mathrm{H}$ signals, retaining only the signals of nonprotonated carbons; otherwise, the plot of $S / S_{0}$ vs. $N t_{\mathrm{r}}$ would be dominated by the initial decay of the C-H signals, 
which interferes with the goal of the experiment to distinguish between different types of nonprotonated carbons based on the strength of their dipolar couplings to protons.

\subsubsection{Saturation-pulse induced dipolar exchange with recoupling (SPIDER)}

\subsubsection{Introduction}

Due to the large linewidths caused by quadrupolar coupling, ${ }^{14}$ N MAS NMR for NOM structure determination has rarely been explored [128], and instead ${ }^{15} \mathrm{~N}$ MAS NMR has been widely exploited [129]. Although ${ }^{15} \mathrm{~N}$ CP/MAS has been used extensively for NOM samples with ${ }^{15} \mathrm{~N}$ in natural abundance $(0.37 \%)$ [28, 130-132], as shown below its sensitivity and its site resolution, for instance between heterocyclic and amide N, are poor and therefore only limited structural information is obtained. A useful alternative is the saturation-pulse induced dipolar exchange with recoupling (SPIDER) technique, which characterizes forms of nitrogen in NOM indirectly via selective detection of ${ }^{13} \mathrm{C}$ bonded to $\mathrm{N}$ [115]. The assignments are more straightforward because ${ }^{13} \mathrm{C}$ chemical shift ranges, for instance of heterocyclic ( 130-155 ppm) and amide C-N (171-176 ppm), have less overlap.

Although the transfer of populations in double resonance (TRAPDOR) or rotational-echo adiabatic-passage double-resonance (REAPDOR) experiments [133, 134] allow for the

selective detection of carbons near ${ }^{14} \mathrm{~N}$, their useful applications, especially in the NOM field, have been lacking. This is due to the requirement in both these experiments for very restrictive high radio-frequency field strengths and low spinning frequencies. The SPIDER technique can select the signals of carbons bonded to nitrogen in complex NOM at spinning frequencies that result in significant centerband intensities even for functional groups with large ${ }^{13} \mathrm{C}$ CSAs, e. g. aromatic carbons. The technique is similar to the relaxation-induced dipolar exchange with 
recoupling (RIDER) technique [135], and achieves recoupling of heteronuclear dipolar couplings by using a series of $\pi$ pulses, such as REDOR $[122,136]$. A train of short ${ }^{14} \mathrm{~N}$ pulses at the center of a sequence of ${ }^{13} \mathrm{C} \pi$-pulses spaced by $\mathrm{t}_{\mathrm{r}} / 2$ is used to saturate ${ }^{14} \mathrm{~N}-{ }^{13} \mathrm{C}$ coherences. Furthermore, by using a $5 \mathrm{kHz}$ spinning frequency and moderate radio-frequency strengths, this technique results in efficient detection of carbons bonded to nitrogen.

\subsubsection{Theoretical background}

The density operator after cross polarization from ${ }^{1} \mathrm{H}$ at the beginning of the SPIDER pulse sequence [115] corresponds to the transverse magnetization:

$$
\rho(0)=S_{x}=S_{x}\left(1-L_{z}^{2}\right)+S_{x} L_{z}^{2}
$$

where $\mathrm{S}$ represents ${ }^{13} \mathrm{C}$ (spin $1 / 2$ ) and $\mathrm{L}$ represents ${ }^{14} \mathrm{~N}$ (spin 1). After $\mathrm{N} / 2$ rotation periods with recoupled dipolar excitation of heteronuclear coherences by a train of ${ }^{13} \mathrm{C}$ pulses spaced by $t_{\mathrm{r}} / 2$, this term becomes

$$
\rho\left(\mathrm{Nt}_{\mathrm{r}} / 2\right)=\mathrm{S}_{\mathrm{x}}\left(1-\mathrm{L}_{\mathrm{z}}^{2}\right)+\mathrm{S}_{\mathrm{x}} \mathrm{L}_{\mathrm{z}}^{2} \cos \mathrm{N} \Phi+\mathrm{S}_{\mathrm{y}} \mathrm{L}_{\mathrm{z}} \sin \mathrm{N} \Phi
$$

where $\Phi$ is the phase acquired during half a rotation period, $\mathrm{t}_{\mathrm{r}} / 2$, under the influence of the ${ }^{14} \mathrm{~N}-{ }^{13} \mathrm{C}$ dipolar interaction.

After complete saturation of the L terms in eq.(16) by a long train of short pulses [115], the density operator is reduced to $S_{x}$, which afterwards evolves again as given in eq.(16) during the reconversion period, so that $\rho\left(\mathrm{Nt}_{\mathrm{r}}+\mathrm{t}_{\mathrm{sat}}\right)=\rho\left(\mathrm{Nt}_{\mathrm{r}} / 2\right)$. The observable SPIDER signal $\mathrm{S}$ is 
obtained by taking the double trace $\operatorname{tr}_{\mathrm{L}, \mathrm{S}}\left[\rho\left(\mathrm{Nt}_{\mathrm{r}}+\mathrm{t}_{\mathrm{sat}}\right)\left(\mathrm{S}_{\mathrm{x}}+\mathrm{i} \mathrm{S}_{\mathrm{y}}\right)\right]$ using the $\mathrm{S}=1 / 2$ and $\mathrm{L}=1$ matrix representations [26], with $\operatorname{tr}_{\mathrm{L}}\left(\mathrm{L}_{\mathrm{z}}\right)=0$ and $\operatorname{tr}_{\mathrm{L}}\left(\mathrm{L}_{\mathrm{z}}^{2}\right) / \operatorname{tr}_{\mathrm{L}}(1)=2 / 3$.

By using the signal without ${ }^{14} \mathrm{~N}$ irradiation as the reference intensity $\mathrm{S}_{0}$, the normalized dephasing $\Delta \mathrm{S} / \mathrm{S}_{0}$ after ${ }^{14} \mathrm{~N}$ complete saturation is

$$
\Delta \mathrm{S} / \mathrm{S}_{0}=\left(\mathrm{S}_{0}-\mathrm{S}\right) / \mathrm{S}_{0}=2 / 3-2 / 3\langle\cos \mathrm{N} \Phi\rangle
$$

By taking into account incomplete ${ }^{14} \mathrm{~N}$ saturation, the final generalized dephasing value for long dephasing times is:

$$
\Delta \mathrm{S} /\left.\mathrm{S}_{0}\right|_{N t_{r} \rightarrow \infty}=2 / 3-1 / 3\left[d+d^{2}\right]
$$

where $d$ refers to incomplete ${ }^{14} \mathrm{~N}$ saturation with $d=\cos ^{\mathrm{n}} B_{1} t_{\mathrm{p}}$ for a series of $\mathrm{n}$ broadband pulses of duration $t_{\mathrm{p}}$.

\subsection{Identification of specific functional groups}

To more clearly demonstrate our specialized analyses for specific functional groups, in the

following sections we will first examine the typical broad ${ }^{13} \mathrm{C}$ solid-state NMR spectra of NOM (Fig. 1). From downfield (higher ppm) to upfield (lower ppm), band by band, we demonstrate how we can identify specific functional groups using a combination of different spectral-editing techniques.

\subsubsection{Ketones and aldehydes (220-185 ppm)}

1. Aldehydes, $-((\mathrm{CH})=\mathrm{O})-$

2. Ketones, $-(\mathrm{C}=\mathrm{O})$ - 
A simple $\mathrm{CP} / \mathrm{TOSS}$ experiment with a short $\mathrm{CP}(50 \mu \mathrm{s})$ can select the signals of aldehydes $(\mathrm{HC}=\mathrm{O})$, because they are protonated, while suppressing those of ketones, since they are not protonated (Fig. 9). Short CP permits only carbons with a directly bonded, strongly dipolar coupled ${ }^{1} \mathrm{H}$ enough time to obtain magnetization. Aldehydes are the only protonated carbons resonating at $>180 \mathrm{ppm}$. Conversely, after dipolar dephasing the signals of ketones are selectively retained.

\subsubsection{Carboxylic acids, carboxylates, esters, and amides (185-165 ppm)}

There are primarily four kinds of functional groups in this region: weakly acidic $-\mathrm{COOH}$, strongly acidic $-\mathrm{COO}^{-}$, ester $-\mathrm{COO}-\mathrm{C}-$, and amide $-(\mathrm{C}=\mathrm{O})-\mathrm{NH}$. These are important functionalities that influence acidity, molecular conformation and its $\mathrm{pH}$ dependence, as well as nutrient or heavy-metal binding in very different ways. With a combination of techniques, all four types of functionalities can be distinguished as follows.

3. $\mathrm{COOH}$ carboxylic acid functionalities can be identified in two-dimensional (2D) ${ }^{1} \mathrm{H}-{ }^{13} \mathrm{C}$ heteronuclear correlation (HETCOR) spectra by the characteristically high ${ }^{1} \mathrm{H}$ chemical shift of $>9$ ppm of the acidic protons. The HETCOR spectra shown in Fig. 10 demonstrate the absence of $\mathrm{COOH}$ groups in insoluble NOM ("humin") but their presence in the corresponding alkalisoluble humic acid fraction.

4. COO-C ester groups typically have chemical shifts $<170 \mathrm{ppm}$. Even more distinctively, their chemical shift anisotropies have a sign opposite to that of all other $\mathrm{COO}$ and $\mathrm{CON}$ groups. We have developed a robust two-dimensional NMR technique, undistorted powder patterns by effortless recoupling (SUPER), for reliably measuring these anisotropies [137] (Figs. 11 and 12). 
5. $\mathrm{C}=\mathrm{O}-\mathrm{NH}$ : Peptide groups, which overlap significantly with the $\mathrm{COO}^{-}$groups, can be distinguished reliably by their close proximity to ${ }^{14} \mathrm{~N}$. Their signals can be selectively obtained by the SPIDER method [115], which detects carbons directly bonded to nitrogen (Fig. 13).

6. $\mathrm{COO}^{-}$groups can often be distinguished from $\mathrm{COOC}$ ester groups based on their typical chemical shifts $>173 \mathrm{ppm}$ and $<170 \mathrm{ppm}$, respectively, and even more reliably based on the position of the $\sigma_{22}$-peak of the CSA powder pattern (see Fig.12).

\subsubsection{Aromatic carbons bonded to heteroatoms (165-145 ppm)}

7. H-bonded phenol protons near $11 \mathrm{ppm}$ are visible in the HETCOR spectra of many NOM samples, in particular if they are rich in lignin-derived residues. Phenols can be identified by 2D HETCOR with OH/NH suppression.

8. Aromatic ethers have often been grouped together with phenols, even though their chemical reactivity is very different. The ethers can be identified by strong cross peaks with $\mathrm{OCH}_{n}$, particularly $\mathrm{OCH}_{3}$ protons near $3.5 \mathrm{ppm}$, in HETCOR spectra.

9. Aromatic esters (aromatic C-O-CO) have chemical shifts $<168$ ppm and a characteristically inverted sign of the CSA, as detected by SUPER NMR [137].

10. Pyridinic N-C groups (in 6-ring heterocycles) can be identified in SPIDER spectra (Fig. 13), with ${ }^{13} \mathrm{C}$ signals at around $150 \mathrm{ppm}$. If the pyridinic carbons are protonated, $\mathrm{N}-\mathrm{CH}$, they also stand out in $\mathrm{CH}_{\mathrm{n}}$-selective spectra obtained with short $\mathrm{CP}$ and dipolar dephasing difference (see above), since $\mathrm{CH}$ resonances near $150 \mathrm{ppm}$ are rare (Fig. 8 (1) and (m)).

\subsubsection{Other aromatics and olefinics (145-95 ppm)}

11. Nonprotonated aromatics can be selected by standard dipolar-dephasing methods, 
which have been adapted for quantitative fast-MAS ${ }^{13} \mathrm{C}$ NMR by applying simple dipolar recoupling principles [47].

12. Signals of char aromatic carbons can be selected based on their long distance from the nearest proton, using recoupling of $\mathrm{C}-\mathrm{H}$ dipolar interactions as described above [114]. Lignin signals can be suppressed to $<3 \%$ while char resonances remain at a high level of $\sim 50 \%$ at a dephasing time of $\sim 0.9 \mathrm{~ms}$.

13. Nitrogen-bonded aromatics can be identified by SPIDER experiments, which provide selective spectra of ${ }^{14} \mathrm{~N}$-bonded ${ }^{13} \mathrm{C}$ sites [115] (Fig. 13).

14. Exomethylenes $\left(\mathrm{C}=\mathrm{CH}_{2}\right)$, which resonate near $125 \mathrm{ppm}$, can be identified using three-

spin coherence selection of $\mathrm{CH}_{2}$ signals, as they are the only $s p^{2}$-hybridized $\mathrm{CH}_{2}$ groups found in NOM. They have been observed, for instance, in gilsonite bitumen collected from the Uinta Basin in Utah (Fig. 14).

\subsubsection{Separation of anomeric from aromatic carbons (115-95 ppm)}

\section{O-CHR-O}

16. O-CRR'-O

\section{Aromatic C-C-O}

The question of whether, or to what extent, the ${ }^{13} \mathrm{C}$ signals between 95 and $112 \mathrm{ppm}$ are due to alkyl O-C-O typical of sugar rings or instead to aromatic carbons bonded to an oxygenbonded carbon has been difficult to answer convincingly by traditional solid-state as well as solution NMR. Such aromatics occur prominently in NOM, e.g., in lignin and tannin residues, where aromatic chemical shifts down to $95 \mathrm{ppm}$ are observed. As a result, reported NMR aromaticities based on simple integration are often incorrect. Separation of these overlapping 
species has been achieved with good efficiencies by the relatively simple CSA filtering technique described above, which takes advantage of the systematic difference in the size of the CSA of $s p^{3}$-hybridized anomeric and $s p^{2}$-hybridized aromatic carbons. This approach can be combined with other spectral-editing techniques such as dipolar dephasing, so that protonated $\mathrm{O}-\mathrm{CH}-\mathrm{O}$, nonprotonated $\mathrm{O}-\mathrm{C}-\mathrm{O}$, protonated aromatics, and nonprotonated aromatics can all be separated.

\subsection{6. $O$ - and $N$-alkyl groups (112-52 ppm)}

18. $\mathrm{OCH}$ groups, occurring prominently as $\mathrm{C}-\mathrm{C}(\mathrm{H}, \mathrm{OH})-\mathrm{C}$ in carbohydrates, are common in most NOM samples. Their signals can be separated from overlapping $\mathrm{OCH}_{2}$ resonances by dipolar-DEPT C-H spectral-editing [113].

19. $\mathrm{OCH}_{2}$ signals can be selected by $\mathrm{CH}_{2}$ spectral-editing techniques such as three-spin coherence selection [85].

20. $\mathrm{OCH}_{3}$ signals can be selected by dipolar dephasing, due to the motional averaging of the $\mathrm{C}-\mathrm{H}$ dipolar couplings by the rotational jumps of the methyl group. In the absence of other motions, methyl signals typically survive dipolar dephasing at a level of $57 \%$. The $\mathrm{OCH}_{3}$ groups in lignin show a characteristic sharp ${ }^{13} \mathrm{C}$ NMR peak at $55 \mathrm{ppm}$, and a cross peak to aromatic protons in ${ }^{1} \mathrm{H}_{-}{ }^{13} \mathrm{C}$ HETCOR spectra. They are found to be preserved in many peat humic acids.

21. OC (quaternary) carbons, bonded to one oxygen and three carbon atoms, appear to be more common in humic and fulvic acids than are other (i.e. non-oxygen-bonded) $\mathrm{sp}^{3}$ hybridized quaternary carbons. In dipolar dephased spectra, a broad peak between 80 and 90 ppm is characteristic for these sites. 
22. NCH typical of peptides can be identified by the SPIDER method, which provides selective spectra of ${ }^{14} \mathrm{~N}$-bonded ${ }^{13} \mathrm{C}$ sites [115]. $\mathrm{NCH}$ groups also produce a prominent peak near 53 ppm in the CH-only spectra obtained by the dipolar DEPT method ([113]; see Fig. 8 $(\mathrm{k}))$.

\subsubsection{Nonpolar aliphatics (52-0 ppm).}

In the aliphatic region, three peaks and several shoulders are observed quite consistently:

23. C- $\underline{\mathrm{CH}}$ signals can be selected by $\mathrm{CH}$-only spectral editing.

24. C- $\mathrm{CH}_{2}$-C signals can be selected by $\mathrm{CH}_{2}$-only spectral editing.

25. $\left(-\mathrm{CH}_{2}-\right)_{\mathrm{n}}$ chains show a characteristic doublet of relatively sharp peaks at 33 and 31 ppm, from crystalline and amorphous polymethylene domains.

26. $\mathrm{CH}_{3}$ groups resonate at $<35 \mathrm{ppm}$ and can be selected by dipolar dephasing, due to the reduction of $\mathrm{C}-\mathrm{H}$ dipolar couplings by fast methyl-group rotational jumps.

27. $\mathrm{C}_{\mathrm{q}}$, quaternary aliphatic carbons, can be selected in dipolar-dephasing experiments.

In summary, using the techniques described above, at least 27 different functional groups can be identified in ${ }^{13} \mathrm{C}$ NMR spectra of complex NOM. Figure 15 summarizes the chemicalshift positions of 36 chemical moieties relevant in NOM that can be resolved based on their characteristic chemical shifts and spectral editing. By contrast, fewer than 10 functional groups were previously distinguished in the literature based on simple, routine ${ }^{13} \mathrm{C} C \mathrm{C} / \mathrm{MAS}$ spectra. Using spectral-editing experiments plus quantitative ${ }^{13} \mathrm{C}$ DP/MAS NMR, most of these 36 functional groups can be quantified. 


\section{Solid-state NMR techniques to investigate organic nitrogen in NOM}

The nitrogen $(\mathrm{N})$ cycle involves multiple biogeochemical transformations of nitrogen and nitrogen-containing compounds in nature. Nitrogen is essential for many biological processes and is crucial for life on earth. For example, N-containing amino acids are the building blocks of proteins, and $\mathrm{N}$ is a key element of the bases in DNA and RNA that make up the genetic code of all life. Therefore, the $\mathrm{N}$ cycle is one of the most important topics in biogeochemistry. The major form of nitrogen in soils and sediments is organic $\mathrm{N}[138,139]$. However, a significant proportion of organic $\mathrm{N}$ in $\mathrm{NOM}$ remains uncharacterized due to analytical limitations and the complex nature of NOM. Major insights into the chemical nature of NOM nitrogen have been provided by using solid-state NMR techniques. ${ }^{15} \mathrm{~N}$ CP/MAS has been the primary solid-state NMR technique used for studying organic $\mathrm{N}$ forms [35, 130, 140-145] in organic matter from soil, water, sediment, coal, and kerogen [35, 130, 132, 140-147]. In these studies, secondary amide N (e.g. from polypeptides) usually accounted for more than $80 \%$ of the organic $\mathrm{N}$ observed. Due to the low natural abundance $(0.37 \%)$ of ${ }^{15} \mathrm{~N}$, isotopic enrichment is highly advantageous [143, 148-155]. Although ${ }^{15} \mathrm{~N}$ CP/MAS NMR has gained great popularity, it does not provide a full picture of the distributions of organic $\mathrm{N}$ forms for reasons described below [144, 145], therefore limiting our understanding of formation mechanisms of

refractory organic N. First, ${ }^{15} \mathrm{~N}$ is of low natural abundance and low gyromagnetic ratio, which leads to low sensitivity and prevents the detection of the minor heterocyclic $\mathrm{N}$ component. Second, CP/MAS favors protonated amide $\mathrm{N}$ and as a consequence underestimates nonprotonated heterocyclic N. Third, a significant portion of heterocyclic $\mathrm{N}$ has a large CSA, leading to large sidebands at low spinning frequencies, which reduces the centerband intensity 
and detectability [146], a problem that is further accentuated at higher magnetic fields. Lastly, signals of amide $\mathrm{N}$ and heterocyclic $\mathrm{N}$ potentially overlap.

An alternative solid-state NMR technique, ${ }^{13} \mathrm{C}\left\{{ }^{14} \mathrm{~N}\right\}$ SPIDER, can also investigate the chemical nature of $\mathrm{N}$ in NOM by detecting ${ }^{13} \mathrm{C}$ bonded to nitrogen. This technique takes advantage of the $>99 \%$ abundant ${ }^{14} \mathrm{~N}$ nucleus, and is more sensitive than ${ }^{15} \mathrm{~N}$ NMR because ${ }^{14} \mathrm{~N}-{ }^{13} \mathrm{C}$ pairs are about three times more abundant than ${ }^{15} \mathrm{~N}$ nuclei and the ${ }^{13} \mathrm{C}$ nucleus has a higher gyromagnetic ratio. Furthermore, the ${ }^{13} \mathrm{C}$-based technique is more attractive for site identification, because ${ }^{13} \mathrm{C}$ NMR signals are more readily assigned and are accessible to welldeveloped spectral-editing techniques. The ${ }^{13} \mathrm{C}\left\{{ }^{14} \mathrm{~N}\right\}$ SPIDER technique has been used to investigate organic $\mathrm{N}$ in humic substances from soil [156] and water [157]. For example, SPIDER analysis of a humic acid fraction from a paddy soil at the International Rice Research Institute in the Philippines [156] indicated that $\mathrm{N}$ covalently bonded to nonprotonated aromatic C, see Fig. 13, as part of a polyphenol-derived anilide compound, had been incorrectly identified as amide $\mathrm{N}$ by ${ }^{15} \mathrm{~N}$ NMR [147]. Thus, both techniques should be used in combination to elucidate the structures of organic $\mathrm{N}$ forms in SOM.

$\mathrm{A}{ }^{15} \mathrm{~N}$ dipolar dephasing technique was also developed to detect nonprotonated and mobile nitrogen functional groups using a recoupled dipolar dephasing time of $291 \mu$ s [158]. This technique has been applied successfully to a fulvic acid from Antarctica [116], and to gilsonite bitumen which is found to contain only protonated organic $\mathrm{N}$ forms [159].

The following types of $\mathrm{N}$ in NOM can be detected using ${ }^{15} \mathrm{~N}$ CP/MAS and more advanced solid-state NMR techniques such as ${ }^{13} \mathrm{C}\left\{{ }^{14} \mathrm{~N}\right\}$ SPIDER: 
(1) Pyridine-like nonprotonated $\mathrm{N}$ in 6-membered rings without $\mathrm{O}$ - or $\mathrm{N}$-substituents, as well as $\mathrm{N}$ in imines (both of these types being $\mathrm{C}=\mathrm{N}-\mathrm{C}$ fragments) have quite characteristic downfield ${ }^{15} \mathrm{~N}$ chemical shifts ( 300 to $450 \mathrm{ppm}$ from external liquid ammonia). However, it is difficult to detect $\mathrm{C}=\mathrm{N}-\mathrm{C}$ by ${ }^{15} \mathrm{~N}$ CP/MAS due to its slow cross polarization and large chemical-shift anisotropy. Its neighboring $\mathrm{sp}^{2}$-hybridized carbon resonates between 145 and $160 \mathrm{ppm}$ and can be detected in SPIDER experiments (Fig. 8 (l) and (m)). If unsubstituted, it can alternatively be identified with excellent efficiency by simple $\mathrm{CH}_{\mathrm{n}}$ selection, since it is the only type of protonated carbon resonating this far downfield.

(2) Imine protonated $\mathrm{N}(\mathrm{C}=\mathrm{N}-\mathrm{H})$ is easily identified as the only type of protonated $\mathrm{N}$ resonating near $300 \mathrm{ppm}$ in the ${ }^{15} \mathrm{~N}$ CP/MAS spectrum.

(3) Pyridine-like nonprotonated $\mathrm{N}$ in 6-membered rings two bonds away from $\mathrm{O}-$ or $\mathrm{N}$ substituents (outside the ring) resonates between 200 and $300 \mathrm{ppm}$ in the ${ }^{15} \mathrm{~N}$ CP/MAS spectrum. Again, its neighboring $\mathrm{sp}^{2}$-hybridized carbon, resonating between 145 and 160 ppm and often protonated, can be detected using SPIDER NMR. The presence of such a structure is indicative of 6-ring aromatic heterocycles (such as adenine).

(4) Pyridine-like nonprotonated $\mathrm{N}$ in 5-membered rings $(\mathrm{C}=\mathrm{N}-\mathrm{C})$, as in the imidazole ring (neutral form) of histidine, also resonates between 200 and $300 \mathrm{ppm}$ in the ${ }^{15} \mathrm{~N}$ CP/MAS spectrum, but can be distinguished from 6-membered rings by the upfield $(<140 \mathrm{ppm}){ }^{13} \mathrm{C}$ resonances of the N-bonded carbons detected in SPIDER NMR.

(5) Protonated $\mathrm{N}$ bonded to $\mathrm{C}=\mathrm{O}$ and another $\mathrm{sp}^{2}$-hybridized carbon in 5 or 6 -membered rings, as in imides $(\mathrm{O}=\mathrm{C}-(\mathrm{NH})-\mathrm{C}=\mathrm{O})$ and unsaturated lactams $\left(\mathrm{C}_{\mathrm{sp} 2} \mathrm{H}-(\mathrm{NH})-\mathrm{C}=\mathrm{O}\right)$, resonates between 140 and $200 \mathrm{ppm}$ in the ${ }^{15} \mathrm{~N}$ CP/MAS spectrum; thymine and guanine are examples. 
The $\mathrm{C}=\mathrm{O}$ resonances detected in SPIDER NMR, e.g. 151-161 ppm for $\mathrm{N}-(\mathrm{C}=\mathrm{O})-\mathrm{N}$ in cyclic ureas, are often distinguishable from those of peptide $\mathrm{C}=\mathrm{O}$.

(6) Pyrrole-like nonprotonated $\mathrm{N}$ in 5-membered rings resonates between 220 and $150 \mathrm{ppm}$ in the ${ }^{15} \mathrm{~N}$ CP/MAS spectrum.

(7) Pyrrole and indole protonated $\mathrm{N}$ in 5-membered rings resonates between 160 and 130 ppm in the ${ }^{15} \mathrm{~N}$ CP/MAS spectrum; the two neighboring aromatic carbons, detected in SPIDER NMR, enable a clear distinction to be made from amide groups and imides.

(8) Anilide nitrogen (nonprotonated- $\mathrm{C}_{\mathrm{sp} 2}-\mathrm{NH}-\mathrm{C}=\mathrm{O}$ ), discussed above, resonates near 132 ppm in the ${ }^{15} \mathrm{~N}$ CP/MAS spectrum, and is bonded to nonprotonated aromatic (distinct from lactams) and $\mathrm{C}=\mathrm{O}$ carbons detected in SPIDER NMR.

(9) Secondary amide $\mathrm{O}=\mathrm{C}-\mathrm{NH}-\mathrm{R}$ in peptides, $\mathrm{N}$-acetyl groups, and saturated lactams, resonates from 110 to $150 \mathrm{ppm}$ in the ${ }^{15} \mathrm{~N}$ CP/MAS spectrum; SPIDER NMR can identify them as bonded to $\mathrm{C}=\mathrm{O}$ or alkyl $\mathrm{CH}$.

(10) Primary amide $\mathrm{O}=\mathrm{C}-\mathrm{NH}_{2}$ as in glutamine resonates at $105-120 \mathrm{ppm}$ in the ${ }^{15} \mathrm{~N}$ CP/MAS spectrum; in SPIDER NMR, the absence of the alkyl C-H signal distinguishes them from secondary amides.

(11) Aromatic primary amines $\left(\mathrm{C}_{\text {arom }}-\mathrm{NH}_{2}\right)$, including anilines, resonate at $50-100 \mathrm{ppm}$ in the ${ }^{15} \mathrm{~N}$ CP/MAS spectrum.

(12) Aliphatic primary amines $\left(\mathrm{CH}_{2}-\mathrm{NH}_{2}\right)$ resonate at $0-60 \mathrm{ppm}$ in the ${ }^{15} \mathrm{~N} \mathrm{CP} / \mathrm{MAS}$ spectrum.

(13) Aliphatic primary ammonium ions $\left(\mathrm{CH}_{2}-\mathrm{NH}_{3}{ }^{+}\right)$resonate at 20-70 ppm in the ${ }^{15} \mathrm{~N}$ CP/MAS spectrum.

\section{Connectivities and proximities}


NMR can provide structural information that goes beyond the individual chemical segments discussed so far, because ${ }^{1} \mathrm{H}-{ }^{13} \mathrm{C}$ dipolar couplings can provide information about internuclear distances on a $6-\AA$ scale. $2 \mathrm{D}{ }^{1} \mathrm{H}_{-}{ }^{13} \mathrm{C}$ HETCOR NMR is a valuable tool for characterizing the environments of carbon sites [160-164].

Close proximity of different functional groups can be observed using 2D HETCOR and 2D HETCOR with dipolar dephasing. In this way, junctions between different groups (e.g., aromatic and alkyl) and larger structural units can be identified. 2D HETCOR is especially useful for the identification of the nearest protons for nonprotonated carbons such as $\mathrm{COO} / \mathrm{NC}=\mathrm{O}[165]$.

\section{Heterogeneities and domains}

\subsection{Introduction to heterogeneities and domains}

The nanometer-scale chemical heterogeneity of NOM is an important structural aspect of complex organic systems. In particular, it can provide insights into the mechanism for NOM formation: partial survival of biomacromolecules (lignin, polysaccharides, proteins, protective coatings, etc.) would result in heterogeneities on a > 1-nm scale, while depolymerizationrepolymerization could lead to a more intimate mixing of components. Domain information is also important for the sorption of organic contaminants because components of NOM segregated into sufficiently large domains can act independently as sorbents [109, 166]. Therefore, domain segregation can potentially be a source of specificity and nonlinearity of sorption, i.e. a nonlinear increase in the sorbed contaminant concentration in NOM as a function of the aqueous concentration of the contaminant [109]. 
There are two types of domains that are relevant here: chemical and physical. The domains of crystalline and amorphous poly(methylene) are good examples of physical domains. Chemical domains refer to similar kinds of chemical compounds spatially segregated from others, for example, a polysaccharide domain or a lignin domain. Usually, the terminology "domain" is used if its diameter is more than $5 \mathrm{~nm}$, whereas "heterogeneity" is chosen for diameters less than $5 \mathrm{~nm}[26] .{ }^{1} \mathrm{H}$ spin diffusion, mediated by ${ }^{1} \mathrm{H}-{ }^{1} \mathrm{H}$ dipolar couplings, proceeds with a diffusion coefficient of 0.2 to $0.8 \mathrm{~nm}^{2} / \mathrm{ms}$ [163]. Within mixing times up to $500 \mathrm{~ms}$, structures on a scale up to $50 \mathrm{~nm}$ can be probed $[26,167,168] .{ }^{1} \mathrm{H}$ is abundant in NOM and proton-proton dipolar couplings are strong, so ${ }^{1} \mathrm{H}$ spin diffusion process is most useful in the NOM system. It provides information on the domain sizes or heterogeneities: for small domains the equilibration of magnetization, observed in a ${ }^{1} \mathrm{H}$ or (after $\left.\mathrm{CP}\right){ }^{13} \mathrm{C}$ spectrum, is faster than for large domains.

\subsection{Techniques for identifying proximities, heterogeneities and domains}

\subsubsection{Goldman-Shen ${ }^{1} H$ spin diffusion with ${ }^{13}$ C detection}

Similar to NMR experiments for measuring exchange, a pulse sequence for probing spin diffusion is composed of three periods: selection, mixing time $t_{m}$, and detection. To start a spin diffusion process, it is usually necessary to begin by creating a spatially inhomogeneous distribution of $z$-magnetization. The ideal case is to retain the magnetization of one component as the source magnetization and to suppress the magnetization of all other components. Then, when spin diffusion starts, the selected magnetization diffuses into its surroundings whose magnetization had previously been eliminated. This is manifested in the spectrum as the reappearance of the initially suppressed signals. Magnetization equilibrates faster for small 
domains than it does for large domains. Therefore, plotting the changes in peak intensities, which reflect the changes in the magnetization, versus $\left(\mathrm{t}_{\mathrm{m}}\right)^{1 / 2}$ provides information on domain sizes or heterogeneities.

In the Goldman-Shen experiment [169], the selection of ${ }^{1} \mathrm{H}$ magnetization is based on differential ${ }^{1} \mathrm{H} \mathrm{T}_{2}$ values, which in turn are due to differences in dipolar couplings. For mobile components, ${ }^{1} \mathrm{H} \mathrm{T}_{2}$ values are longer than for rigid components, due to a reduction of the proton-proton dipolar couplings by motional averaging. This technique uses a three-pulse sequence on ${ }^{1} \mathrm{H}$, followed by cross polarization to ${ }^{13} \mathrm{C}$ for detection with good chemical-shift resolution. The first $\pi / 2$-pulse flips the ${ }^{1} \mathrm{H}$ magnetization to the $x y$-plane. After a short period of time, typically $30 \mu \mathrm{s}$, the magnetization arising from rigid components is dephased by strong ${ }^{1} \mathrm{H}-{ }^{1} \mathrm{H}$ dipolar couplings so that only the magnetization of the mobile components remains. The second pulse then flips the remaining magnetization back to the $z$-axis. During the ensuing mixing time $\mathrm{t}_{\mathrm{m}},{ }^{1} \mathrm{H}$ magnetization from the mobile components diffuses into the rigid regions. The third ${ }^{1} \mathrm{H}$ pulse terminates the mixing time and flips the magnetization into the transverse plane for cross polarization to ${ }^{13} \mathrm{C}$. The intensity increase of the initially suppressed signals from the rigid regions, and the corresponding signal intensity loss of the mobile components indicate how spin diffusion evolved between pulses two and three. Figure 16 shows the result of a Goldman-Shen experiment on a humic acid sample from water in the St. Lawrence Estuary [55]; the mixing times range from $0.4 \mathrm{~ms}$ to $20 \mathrm{~ms}$. Within $5 \mathrm{~ms},{ }^{1} \mathrm{H}$ magnetization from mobile aliphatic chains has reached $\mathrm{OCH}, \mathrm{NCH}, \mathrm{C}=\mathrm{C}$, and $\mathrm{COO} / \mathrm{NC}=\mathrm{O}$ segments, proving their proximity to the mobile acyl chains.

\subsection{2. ${ }^{1} \mathrm{H}$ inversion recovery with ${ }^{13} \mathrm{C}$ detection}


The pulse sequence of the ${ }^{13} \mathrm{C}$-detected proton inversion recovery experiment is ${ }^{1} \mathrm{H} \pi$-pulse $-\mathrm{t}_{\text {recov }}-{ }^{1} \mathrm{H} \pi / 2$-pulse $-\mathrm{CP}$ to ${ }^{13} \mathrm{C}-{ }^{13} \mathrm{C}$-detection $[108,109]$. This sequence probes ${ }^{1} \mathrm{H} \mathrm{T}_{1}$ relaxation, with good structural resolution achieved by ${ }^{13} \mathrm{C}$ detection after cross polarization. Since many NOM samples are heterogeneous, differences in ${ }^{1} \mathrm{H} \mathrm{T}_{1}$ relaxation are expected, and can be used to explore the heterogeneity or domains of NOM. The longest ${ }^{1} \mathrm{H} \mathrm{T}_{1}$ is often observed for the sharp peak of crystalline polymethylene chains at $33 \mathrm{ppm}$. Since ${ }^{1} \mathrm{H}$ spin diffusion averages ${ }^{1} \mathrm{H} \mathrm{T}_{1}$ times with a diffusion coefficient around $0.5 \mathrm{~nm}^{2} / \mathrm{ms}$, differences in ${ }^{1} \mathrm{H} \mathrm{T}_{1}$ indicate domains of more than $10 \mathrm{~nm}$ diameter.

\subsubsection{D HETCOR with spin diffusion}

Heterogeneities and domains can also be analyzed by ${ }^{1} \mathrm{H}$ spin diffusion occurring during a mixing time between ${ }^{1} \mathrm{H}$ evolution and cross polarization to ${ }^{13} \mathrm{C}$ in ${ }^{1} \mathrm{H}^{13} \mathrm{C}$ HETCOR experiments (Fig. 17). Nonequilibrium ${ }^{1} \mathrm{H}$ magnetization is generated during the ${ }^{1} \mathrm{H}$ chemicalshift evolution, since the magnetization in different domains oscillates with a different chemical-shift frequency. The experiment is performed with homonuclear ${ }^{1} \mathrm{H}$ decoupling, for instance by the frequency-switched Lee-Goldburg (FSLG) sequence, which works well at 6.5 kHz MAS. Spin diffusion during cross polarization to ${ }^{13} \mathrm{C}$ can be avoided by short (e.g. $80 \mu \mathrm{s}$ ) Lee-Goldburg cross polarization, which provides ${ }^{1} \mathrm{H}-{ }^{1} \mathrm{H}$ dipolar decoupling [163]. Aromatic and OCH proton resonances have generally been the focus of HETCOR experiments with ${ }^{1} \mathrm{H}$ spin diffusion, because their 3-ppm chemical-shift separation is usually sufficient for obtaining cross peaks with $<20 \%$ contributions arising from wings of strong peaks at other proton chemical shifts. Where necessary, such overlap-induced artifacts can be suppressed by combining HETCOR with a ${ }^{1} \mathrm{H}$ chemical-shift filter that reduces the intensity of the 
undesirable peaks. A series of 2D HETCOR spectra with different spin diffusion mixing times is usually acquired. A typical peat humic acid shows fast aromatic-OCH spin diffusion, reaching 50\% of equilibrium within $0.05 \mathrm{~ms}$, see Fig. 17, indicating mixing of lignin and $\mathrm{OCH}$ components on a monomer length scale. The only larger domains of homogeneous composition are the semicrystalline polymethylene domains discovered by $\mathrm{Hu}$ et al. [170] using the ${ }^{1} \mathrm{H}$ inversion recovery technique.

\subsubsection{Combined rotation and multiple-pulse spectroscopy (CRAMPS) $2 D$ exchange NMR}

The basic principle of exchange NMR is the detection of a slow change in NMR frequency during the mixing time between the measurements of the frequency during evolution and detection periods $[26,171,172]$. For $2 \mathrm{D}$ exchange NMR, if there is no slow dynamics during the mixing time, the frequency remains unchanged and only diagonal signal exists. However, if frequencies change during the mixing time, off-diagonal signals appear. CRAMPS [173] or fast MAS at $>110 \mathrm{kHz}$ achieves high resolution of proton spectra in solids. With finely-tuned CRAMPS and modern robust preamplifiers, or under fast MAS, it is quite straightforward to conduct a ${ }^{1} \mathrm{H}-{ }^{1} \mathrm{H} 2 \mathrm{D}$ exchange experiment, with good sensitivity and resolution. Cross peaks in such an experiment indicate spin exchange between certain sites mediated by ${ }^{1} \mathrm{H}$ dipolar couplings. Figure 18 shows ${ }^{1} \mathrm{H}$ CRAMPS 2D exchange NMR of a whole soil organic matter sample from a rice soil in the Philippines. Four different mixing times were used: $3 \mu \mathrm{s}, 300 \mu \mathrm{s}$,

$1 \mathrm{~ms}$, and $10 \mathrm{~ms}$. At a mixing time of $10 \mathrm{~ms}$, the ${ }^{1} \mathrm{H}$ magnetization is essentially equilibrated throughout the whole structure, except for non-polar aliphatic domains; this indicates that $\mathrm{O}$ alkyl and aromatic groups are in close proximity and the diameter of any O-alkyl or aromatic heterogeneities is $<2 \mathrm{~nm}$. 


\subsubsection{Long-range recoupled dipolar dephasing}

Techniques have also been developed specifically for detecting fused aromatic ring domains. Routine one-bond dipolar dephasing for $40-70 \mu$ s can be used to select signals of nonprotonated carbons and mobile groups such as $\mathrm{CH}_{3}[47,73,174]$. Long-range dipolar dephasing can be employed to select signals of fused ring systems: protons at the periphery of aromatic rings dephase peripheral carbons that are close to them before reducing the signals of interior carbons of fused aromatic rings. Thus, if an appropriate dephasing time is chosen, peripheral carbons in the fused ring systems can be dephased while the interior carbons are retained [114]. To achieve long-range dipolar dephasing, a longer evolution under the $\mathrm{C}-\mathrm{H}$ coupling is required $[30,121,124]$. It has been demonstrated that at a recoupled dipolar dephasing time of $0.9 \mathrm{~ms}$, fused ring $\mathrm{C}$ signals are selected by the dephasing of the signals of all carbons within two bonds of protons [114].

\subsubsection{Principles for selecting different techniques}

In the discussion so far, several techniques have been described that can be used to investigate heterogeneities and domains. In this section, we discuss the basis for selecting particular techniques in different specific situations.

For Goldman-Shen spin diffusion, it is important that both mobile and rigid components with different ${ }^{1} \mathrm{H} \mathrm{T}_{2}$ values are present in the NOM samples; otherwise it is impossible to select the magnetization of one component exclusively. However, because mobile and rigid components have different spin diffusion coefficients, it is relatively difficult to make an

accurate estimate of domain sizes based on this technique. The use of ${ }^{1} \mathrm{H}$ inversion recovery 
technique requires heterogeneous ${ }^{1} \mathrm{H} \mathrm{T}_{1}$ values of different components in NOM samples. Furthermore, this technique can only be applied to samples with domains having diameters more than $10 \mathrm{~nm}$ (for samples with short ${ }^{1} \mathrm{H} \mathrm{T}_{1}$ ) or perhaps even as large as $100 \mathrm{~nm}$ because ${ }^{1} \mathrm{H}$ spin diffusion averages the values of ${ }^{1} \mathrm{H} \mathrm{T}_{1}$ rapidly [102]. 2D HETCOR with spin diffusion is ideal for rigid systems where the selection of the magnetization of one component based on differences in either ${ }^{1} \mathrm{H} \mathrm{T}_{1}$ or $\mathrm{T}_{2}$ values is difficult. The ${ }^{1} \mathrm{H}-{ }^{1} \mathrm{H}$ 2D exchange technique can be an alternative to $2 \mathrm{D}{ }^{1} \mathrm{H}-{ }^{13} \mathrm{C}$ HETCOR with spin diffusion. However, the implementation of this technique is more demanding, and thus, 2D HETCOR with spin diffusion is more routinely used. Finally, ${ }^{1} \mathrm{H}^{13} \mathrm{C}$ long-range dipolar dephasing is used specifically for selecting fused-ring aromatic domains.

\subsection{Examples of close proximities, heterogeneities, and domains}

\subsubsection{Lignin residues}

Lignin was long regarded as the primary source of humic substances and is involved in several proposed pathways of humus synthesis [1]. It was thought that lignin is incompletely degraded by microorganisms and the residues become part of humic substances. According to another hypothesis, under microbial attack phenolic aldehydes and acids are released from lignin and then converted to quinones. These quinones polymerize with or without amino compounds and form humic substances [1].

Using advanced solid-state NMR techniques, it is possible to detect the presence of lignin residues in humic substances. Figure 19 shows the protonated-carbon-only spectra of lignin, a triple-cropped rice soil mobile humic acid (MHA) and a dryland soil MHA from the International Rice Research Institute in the Philippines. The close similarity in the aromatic 
region between the protonated-carbon spectra of lignin and of triple-cropped MHA suggests the presence of lignin in MHA. In contrast, the corresponding spectral features of lignin and dryland MHA are different. ${ }^{1} \mathrm{H}-{ }^{13} \mathrm{C}$ 2D HETCOR can reveal the cross-peak pattern characteristic of lignin (Fig. 20): the correlation between aromatic ${ }^{1} \mathrm{H}$ at $\sim 7 \mathrm{ppm}$ and $\mathrm{OCH}_{3}{ }^{13} \mathrm{C}$ at $\sim 56 \mathrm{ppm}$ and the correlation between aromatic $\mathrm{C}-\mathrm{O}{ }^{13} \mathrm{C}$ at $\sim 150 \mathrm{ppm}$ and $\mathrm{OCH}_{3}{ }^{1} \mathrm{H}$ at $\sim 4$ ppm is clearly seen in the ${ }^{1} \mathrm{H}-{ }^{13} \mathrm{C} 2 \mathrm{D}$ HETCOR spectrum of lignin (Fig. 20 (c)). In order to remove cross-peaks to the $\mathrm{OH}$ and $\mathrm{NH}$ signals and enable unequivocal assignments, ${ }^{1} \mathrm{H}^{13}{ }^{13} \mathrm{C} 2 \mathrm{D}$ HETCOR with OH/NH suppression by ${ }^{1} \mathrm{H}$ chemical shift anisotropy filtering[175], based on the observation that the CSA parameter $|\delta|$ of protons in $\mathrm{C}-\mathrm{H}$ bonds is much less than that of $\mathrm{OH}$ and $\mathrm{NH}$ protons $[117,176]$, has been adopted and applied to a peat humic acid. The characteristic aromatic- $\mathrm{OCH}_{3}$ cross peaks in this $2 \mathrm{D}$ HETCOR spectrum indicate that lignin residues exist in this humic acid.

\subsubsection{Fused-ring aromatic domains}

Despite its importance in the global carbon cycle, black carbon has remained poorly characterized [177]. The signals of fused ring $\mathrm{C}$ characteristic of black carbon can be selected by the recoupled long-range $\mathrm{C}-\mathrm{H}$ dipolar dephasing technique [114], which has been applied to investigate various NOM samples.

Figure 21(a) presents an example of the application of this technique to the IHSS Leonardite HA. The dephasing curve of the aromatic carbons bonded to three carbon neighbors (signal between 107 and142 ppm) for this sample is shown in Fig. 21 (b) and compared with those of IHSS peat humic acid, wood charcoal and lignin. At a dipolar dephasing time of 0.86 
ms, only $17 \%$ of aromatic signals remain for Leonardite HA, compared with $55 \%$ of aromatic signal for wood char.

The results show that the fraction of large char-like clusters of polycondensed aromatic rings is relatively low in Leonardite humic acid. Nevertheless, the aromatics in the humic acid are found to consist of larger or more densely substituted ring systems than those in lignin, which contains only individual benzene rings. The aromatics in these humic acids can consist of only two or three fused rings [114]. Application of this technique to a bituminous coal yields similar sizes of fused rings (unpublished data).

\subsubsection{Nonpolar aliphatic domains}

In some NOM samples, nonpolar aliphatic domains are the only significant domains identified, while coal and kerogen also contain fused-ring aromatic domains [178]. The nonpolar aliphatic domains have been found to be composed of crystalline poly(methylene) and amorphous branched nonpolar aliphatics [109]. The existence of such domains in NOM

has been detected by ${ }^{1} \mathrm{H}$ inversion recovery experiments. The amorphous nonpolar aliphatic domains have been shown to offer an excellent environment for the sorption of phenanthrene by partitioning $[109,170]$.

\section{Segmental dynamics}

\subsection{Importance of information on segmental dynamics}

The properties of NOM are affected both by its chemical and physical structures and by segmental dynamics. For example, it has been proposed that both rubbery and glassy components exist in NOM $[179,180]$. Rubbery regions in NOM with a glass transition 
temperature far below ambient temperature can play important roles as excellent sorption environments for organic contaminants $[179,180]$. Solid-state NMR provides powerful techniques for characterizing NOM dynamics in detail over a wide range of motional rates. Fast segmental mobility with rates around $100 \mathrm{MHz}$ can be investigated based on $\mathrm{T}_{1}$ relaxation-time measurements, unless the effects of unpaired electrons are dominant; the amplitudes of motions with $>100 \mathrm{kHz}$ rates can be characterized by analysis of ${ }^{1} \mathrm{H}$ wideline shapes; and slow dynamics with rates between $1 \mathrm{~Hz}$ and $1 \mathrm{kHz}$ can be examined using exchange NMR experiments.

\section{2. ${ }^{1} \mathrm{H}$ wideline spectra and wideline separation (WISE)}

The widths of proton lines have long been used for assessing the amplitude of fast

molecular motions. ${ }^{1} \mathrm{H}$ NMR spectra of rigid materials are characterized by broad lines due to strong proton-proton homonuclear dipolar couplings, which are averaged by molecular motions leading to reduced line widths $[26,36,41]$. The extreme case is solution NMR, where fast tumbling and diffusion of molecules results in narrow lines centered at the isotropic chemical shifts [108]. Monitoring changes in the ${ }^{1} \mathrm{H}$ line width with temperature can provide information on changes in motional rate and amplitude. Usually, proton spectra acquired using commercial probes are afflicted with background signals from ${ }^{1} \mathrm{H}$ in the material surrounding the NMR coil. A simple pulse sequence has been designed for ${ }^{1} \mathrm{H}$ probehead background suppression [181]. In short, two one-pulse spectra, with pulse flip angles of $\beta$ and $2 \beta$ (e.g. $90^{\circ}$ and $180^{\circ}$ ), are acquired. While the response of ${ }^{1} \mathrm{H}$ spins inside the coil is strongly nonlinear (e.g. an increase of the flip angle from $90^{\circ}$ to $180^{\circ}$ leads to a strong decrease of the signal), it is nearly linear outside the coil since the $B_{1}$ field is very weak. The essentially background-free 
spectrum is obtained by subtracting the second spectrum, scaled by 0.5 to compensate for the doubled flip angle outside the coil, from the first [181]. Due to slight nonlinearity of the response even outside the coil, in practice a slightly larger scaling factor of ca. 0.53 provides the best background suppression.

However, due to the lack of spectral resolution of static ${ }^{1} \mathrm{H}$ spectra, it is virtually impossible to obtain information on molecular dynamics of individual components through this technique. This limitation can be overcome by using the 2D wideline separation (WISE) technique [26, 182]. In a 2D WISE spectrum, the second dimension displays ${ }^{13} \mathrm{C}$ chemical shifts which provide information on specific functional groups, while the first dimension shows ${ }^{1} \mathrm{H}$ wideline shapes. The simplest version of the 2D WISE pulse sequence derives from the standard CP/MAS/TOSS experiment simply by inserting an incremented delay $\mathrm{t}_{1}$ after the initial ${ }^{1} \mathrm{H} 90^{\circ}$ pulse. Fourier transformations over $\mathrm{t}_{1}$ and $\mathrm{t}_{2}$ result in ${ }^{1} \mathrm{H}$ wideline spectra separated according to the corresponding ${ }^{13} \mathrm{C}$ isotropic shifts. In dry humic acids, the line widths of the $\mathrm{OCH}$ protons are consistently larger than those of the aromatic protons, showing that there are no fast, large-amplitude motions of the carbohydrates that would facilitate contaminant sorption by these domains [163]. The reduced line width of the aromatic protons is most likely due to the lower local proton density for aromatics. Lee-Goldburg CP of $200 \mu$ s was used to prevent ${ }^{1} \mathrm{H}$ spin diffusion during $\mathrm{CP}$.

\subsection{Slow dynamics: Centerband-only detection of exchange (CODEX) NMR}

Slow dynamics can also be important for macroscopic properties of macromolecular solids. With the CODEX technique $[118,183]$, slow (with rates between 0.2/s and 2000/s) segmental reorientations of NOM components can be examined with good sensitivity and site resolution. The pulse sequence of CODEX contains the elements of the typical exchange experiment 
outlined above, with a mixing time between two periods of evolution of transverse magnetization $[118,183]$. In brief, after cross polarization the CSA is recoupled by a train of two $\pi$-pulses per rotation period $t_{\mathrm{r}}$, for a duration of $N / 2 t_{\mathrm{r}}$. Subsequently, the magnetization is stored along the $z$-direction for a mixing time $t_{\mathrm{m}}$. During a second period of $N / 2 t_{\mathrm{r}}$ with recoupling of the anisotropic chemical shift, its evolution is refocused into a stimulated echo if no motions occurred during $t_{\mathrm{m}}$; if segmental reorientation during $t_{\mathrm{m}}$ has changed the anisotropic chemical shift, the echo is incomplete and its intensity reduced. The magnetization at the echo maximum, $S$, is stored for a second $z$-period, and finally read out for detection. The second $z$ period enables measurement of a reference spectrum $S_{0}$ without exchange but with the same total $z$-storage duration as was used for recording the signal with exchange, $S$. The normalized intensity, $S / S_{0}$, can be recorded as a function of $t_{\mathrm{m}}$ or $N t_{\mathrm{r}}$. For long $t_{\mathrm{m}}$ and $N t_{\mathrm{r}}, S / S_{0}=1 / M$ is characteristic of symmetric $M$-site jumps. It should be noted that longer mixing times $>100 \mathrm{~ms}$,

other processes such as ${ }^{14} \mathrm{~N}$ longitudinal relaxation induced dipolar exchange or ${ }^{13} \mathrm{C}$ spin diffusion may interfere with detection of motion by exchange NMR experiments such as CODEX.

\subsection{Examples of segmental mobility in NOM - mobile lipids detected in NOM from natural} waters

A mobile aliphatic segment was detected in humic acids from NOM in the Saguenay Fjord and the St. Lawrence Estuary [55]. Figure 22 shows the spectra used to identify the mobile aliphatic segments of the humic acid from the St. Lawrence Estuary; Figures 22(a) and (b) show the ${ }^{13} \mathrm{C}$ CP/TOSS and its dipolar-dephased spectra, respectively. The dipolar-dephased spectrum indicates that this sample contains a significant fraction of mobile aliphatic segments. 
Figure 22(c) shows the DP/MAS spectrum collected using a short recycle delay of $1.5 \mathrm{~s}$, highlighting the mobile segments due to shorter ${ }^{13} \mathrm{C} \mathrm{T}_{1}$ values of mobile groups relative to those of rigid groups. This spectrum shows the signals predominantly of the mobile aliphatic region, a small $\mathrm{COO} / \mathrm{NC}=\mathrm{O}$ band near $173 \mathrm{ppm}$ plus a small peak near $130 \mathrm{ppm}$ assigned to olefinic $\mathrm{CH}=\mathrm{CH}$ groups in mobile acyl chains (Fig. 22(f)). Figure 22(d) displays the expanded aliphatic region between $5-50 \mathrm{ppm}$ of the $1.5-\mathrm{s}{ }^{13} \mathrm{C}$ DP/MAS spectrum showing the signals characteristic of mobile acyl chains, which is confirmed by its proton spectrum (Fig. 22(e)). This component (Fig. 22(f)) has a lipid-like structure with acyl chains of approximately 10 to 20 carbons, typically containing a couple of $\mathrm{C}=\mathrm{C}$ double bonds, and terminated by a $\mathrm{CH}_{3}$ group.

\section{Applications of solid-state NMR to specific types of organic matter}

\subsection{Soil organic matter}

The chemical nature of soil organic matter (SOM) has attracted considerable attention from chemists and soil scientists for many decades. Its chemical structure has not yet been fully identified, mainly due to the convergence of two intractable issues: the complex (and perhaps nonreproducible) chemical nature of SOM, and the inability to fully separate inorganic soil materials (which interfere with NMR and other analyses) from SOM without potentially altering the intrinsic chemical structure and composition of SOM.

Given these challenges, prevailing ideas on SOM structure have historically depended on available technologies for extracting SOM from soil and for its chemical characterization. As described in detail by Waksman [184], Kononova [185], and Stevenson [1], during an era

focused on chemical digestion reactions in the early $20^{\text {th }}$ century, SOM was generally thought 
to be derived primarily from lignin and its reaction with proteins. However, with further studies and improving chromatographic capabilities, by the middle of the $20^{\text {th }}$ century it became clear that intact lignin and whole proteins were scarce in SOM. Hence a new dominant perspective on SOM structure became the "polyphenol theory", in which lignin and proteins are first broken down to their respective monomers, which then recombine to form SOM [1, 185]. As with the previous lignin-protein perspective, this school of thought still presumed SOM to be highly aromatic and composed of unique chemical structures that form solely in soil. A catalyst for this idea may have been the chemical extractions used to isolate SOM from soil for chemical characterization; these often involved strong extractants which might have partially created the aromatic compounds conjectured to dominate SOM, for example the Haworth [186] model of an aromatic core with aliphatic substituent groups.

Movement away from this paradigm was facilitated through the first applications of ${ }^{13} \mathrm{C}$ NMR to SOM characterization The first studies were made in the 1960s and 1970s [35], and a major early finding was that aromatic compounds comprised much lower proportions of total C than had previously been proposed, averaging roughly only about 35\% [1]. Accompanying the decreased abundance of aromatic compounds was a corresponding increase in aliphatic compounds, especially fatty acids and alkanes. Early NMR studies used solution ${ }^{13} \mathrm{C}$ NMR to analyze dissolved humic acid fractions, but by the 1980s high-resolution solid-state ${ }^{13} \mathrm{C}$ NMR with magic-angle spinning and high-power proton decoupling had become available for studying both humic acid fractions and whole SOM $[1,30]$.

NMR observations of relatively high abundance of aliphatic compounds encouraged researchers to expand their discussion of SOM formation from processes solely involving abiotic chemical rearrangements to include also biological processes. Extractions of SOM 
from soil for subsequent analysis started more commonly to use non-chemical means, especially based on density [187], particle size [188] and aggregation [189]. More attention was given to non-chemical factors of SOM stabilization, such as inaccessibility to microbial activity and interactions between chemical recalcitrance and environmental factors including soil mineral surfaces and biological activity [190].

Recent reviews of NMR results have speculated whether SOM structure could be many heterogeneous units that are perhaps cross-linked in an irregular manner that is too complex for enzymatic degradation [191, 192], or perhaps predominantly linked through noncovalent hydrophobic interactions [13]. These small units could comprise a range of identifiable biological compounds derived from plant, animal or microbial sources and possibly also humification end products.

Recently SOM has been claimed to be composed predominantly of molecules of microbial origin [193, 194]. Similarly, its cycling and stabilization would be attributed solely to microbial transformations, while abiotic processes, including chemical condensation, would play a limited to or negligible role. These claims constitute a swing of the pendulum to the opposite - and equally unlikely — extreme of the sole emphasis on chemical processes in the previous century. Claims of a microbial origin of NOM are contradicted by decades of chemical extractions that identified plant residues in SOM ([1, 184], with lignin examples discussed above) and solid-state ${ }^{13} \mathrm{C}$ NMR evidence of a more aromatic nature of SOM than for microbial materials, which can be composed of $75 \%$ carbohydrates in addition to aliphatic compounds including fatty acids and amino acids. For instance, spectra of Iowa Mollisols not subjected to any extraction were dominated by the signals of oxidized char residues [70], 
which are distinct from biomolecules, refuting claims of dominance of microbial residues in SOM.

A case study that illustrates the enhanced capabilities of solid-state NMR to depict SOM structure is our evolving understanding of soil $\mathrm{N}$ forms. Most soil $\mathrm{N}$ is not readily available to crop growth or short-term cycling, and identifying its chemical nature might help explain this recalcitrance. Prior to the use of NMR, soil amino-N forms were identified by $\mathrm{HCl}$ extraction of the soil followed in early years by titration of the acid extracts [195] and later by chromatographic separation of the $\mathrm{HCl}$ extracts $[1,196]$. Typically only $30-40 \%$ of total soil $\mathrm{N}$ was identified as amino $\mathrm{N}$, strengthening the paradigm of complex humic molecules that were unique to soil, as such compounds would tightly hold the unidentified $\mathrm{N}$ against acid extraction or microbial decomposition through covalent binding or transformation into aromatic forms.

By contrast, solid-state ${ }^{15} \mathrm{~N}$ CP NMR studies by Knicker et al. [131] and Knicker and Lüdemann [197] demonstrated an overwhelming dominance of soil $\mathrm{N}$ forms by amide-N. Similarly, Knicker and Hatcher et al. [198] used ${ }^{15} \mathrm{~N}$ CP NMR on soil that had already been extracted by $\mathrm{HCl}$ for amino acids and found the residual, unextracted soil $\mathrm{N}$ to contain amide. This finding has also been confirmed using direct measurement of amino acids in the hydrolyzed residue [199]. Hence the typically poor recovery of soil $\mathrm{N}$ as amino- $\mathrm{N}$ might be due more to the inability to wholly extract $\mathrm{N}$ from soil for amino analysis than to the presence of a large proportion of non-amino $\mathrm{N}$. The accuracy of estimation using ${ }^{15} \mathrm{~N}$ NMR remains under debate, as some proportions of heterocyclic $\mathrm{N}$ have been identified by several means including ${ }^{15} \mathrm{~N}$ NMR $[139,147]$, and significant proportions of soil $\mathrm{N}$ might be heterocyclic $\mathrm{N}$ that is difficult to detect by ${ }^{15} \mathrm{~N}$ CP NMR [144]. Yet the conclusion that much soil $\mathrm{N}$ is in fact 
not aromatic has helped expand studies of SOM cycling and sequestration from a focus on merely abiotic chemical recombinations to also include biological modes of $\mathrm{N}$ stabilization.

While SOM structure still enjoys substantial attention from solid-state NMR researchers, other useful NMR applications would address SOM function in soil behavior: can we identify chemical traits of SOM that determine its involvement in soil processes and agroecosystem sustainability? More accurate identification of these compounds could help improve land management by fostering accumulation of favorable compounds or decomposition of unfavorable ones. In addition to the examples of pesticide adsorption described above, the previously mentioned SPIDER study by Schmidt-Rohr et al. [156] verified the existence of aromatic-bonded $\mathrm{N}$ forms in a rice soil SOM and associated their accumulation with lignin residues, which had been hypothesized to bind soil organic $\mathrm{N}$ into unavailable forms and cause long-term declines in grain yield. Also, Mao et al. [200] distinguished amino C from overlapping methoxyl and carboxyl $\mathrm{C}$ signals to more accurately depict the benefits of animal manure application to soil $\mathrm{N}$ accumulation and crop $\mathrm{N}$ supply. More accurate measurement of specific functional groups would also help NMR complement current chromatographic techniques that can follow the cycling of specific organic compounds or even isotopically labeled compounds-but only in extracts of the samples. By contrast, solid-state NMR can detect functional groups throughout the SOM of the original, unextracted samples. This advantage seems especially notable when studying soil processes that can involve multiple functional groups or compounds, including pesticide absorption [201] and soil C sequestration [202].

\subsection{Aquatic organic matter}


Organic matter in the aquatic systems exists in a continuum of sizes, including dissolved molecules and colloids as well as suspended and sinking particles. Their separation from natural waters is primarily based on passage through filters and membranes with varying pore sizes [203]. Dissolved organic matter (DOM) and particulate organic matter (POM) are operationally defined as the fractions that are passed and retained, respectively, on $0.45-\mu \mathrm{m}$ pore size filters. Dissolved organic matter typically represents $\sim 60 \%$ and $>95 \%$ of the total organic carbon (OC) load in rivers and oceans, respectively [204], while POM accounts for a minor fraction of total $\mathrm{OC}$ in the aquatic systems, and is further divided into sinking POM $(\sim 100-1000 \mu \mathrm{m})$ and suspended POM $(\sim 0.1-60 \mu \mathrm{m})$ [205]. NMR spectroscopy is arguably the most powerful tool for comprehensively characterizing complex organic matter. ${ }^{13} \mathrm{C},{ }^{1} \mathrm{H},{ }^{15} \mathrm{~N}$ and less frequently ${ }^{31} \mathrm{P}$ are the main nuclei involved in NMR studies of POM and DOM,

though ${ }^{1} \mathrm{H}$ and ${ }^{31} \mathrm{P}$ NMR are exclusively or mostly performed in solution state. NMR studies of DOM have been the topic of several recent reviews $[18,19,206]$. The majority of NMR-based DOM research requires DOM concentration and isolation to obtain a solid prior to NMR analysis, even for solution NMR. This is usually achieved by methods such as solid-phase extraction, reverse osmosis coupled with electrodialysis, or ultrafiltration, followed by freezedrying or rotary evaporation to remove the solvent. In this section, the application of solid-state NMR to aquatic organic matter will be reviewed selectively.

\subsubsection{Solid-state ${ }^{13}$ C NMR applications}

One-dimensional solid-state ${ }^{13} \mathrm{C}$ NMR provides information on the average functional group distribution of carbon in aquatic OM. Solid-state NMR has been used to study spatial and temporal (seasonal) variations of DOM composition [205, 207-218], to compare the 
structure of DOM and its potential sources (diatom extracellular material, algae precursor, and plant/soil leachate) $[157,219,220]$, to characterize specific fractions of DOM [221-225], as well as to examine photochemically induced changes in DOM composition [226-230]. Study of aquatic POM by solid-state NMR is much less common than that of DOM. Among these studies, ${ }^{13} \mathrm{C}$ CP/MAS NMR has been applied to compare different size fractions of aquatic OM including particulate and colloidal organic material and dissolved fulvic acid [209], show structural changes of POM with depth [50, 205, 231, 232] or difference sources [55], and characterize the nonhydrolyzable fraction of sinking POM [233].

In most studies ${ }^{13} \mathrm{C}$ NMR spectra were acquired using CP/MAS, occasionally ramp CP/MAS [211, 225], and less frequently with quantitative DP/MAS [55, 157, 218, 231, 232]. Advanced solid-state NMR techniques such as spectral editing and 2D HETCOR with or without spin diffusion have also been applied to resolve broad and overlapping resonances and reveal proximities of functional groups, respectively, providing numerous structural details rarely reported in non-NMR geochemical studies [55, 116, 157, 218, 229-232]. For instance, Mao et al. [218] proposed that nonprotonated O-alkyl carbons, which were identified by dipolar dephasing editing and found in DOM in significant amounts, could be used as a humification index. In addition, the supramolecular structure of humic acids from sinking POM as deduced from NMR spin diffusion data (mobile lipids in close proximity to peptides and carbohydrates) resembled that of bacterial cell walls [55], indicating a major bacterial contribution. Though Hedges et al. [234] found minimal changes in bulk functional group composition between surface plankton and marine sinking POM based on ${ }^{13} \mathrm{C} C P / M A S$, by using quantitative DP/MAS and spectral editing Mao et al. [232] noted clear variations of 
different functional moieties in POM as a function of depth, supporting the selective degradation (or preservation) theory.

\subsubsection{Solid-state ${ }^{15}$ N NMR applications}

Several studies have used solid-state ${ }^{15} \mathrm{~N}$ CP/MAS NMR to characterize the forms of nitrogen present in aquatic OM [132, 218, 222, 235-238]. Collectively these studies indicate that the majority of nitrogen is in the amide form, while amines and heterocyclic nitrogen are negligible or much less abundant. The relative proportions of these different nitrogen functionalities were shown to vary among different DOM fractions [222]. The dominant amide signals may arise from peptide bonds in proteins that are considered labile, as well as from more stable biopolymers such as chitin and peptidoglycan with amide side chains. To better define the chemical forms of amide nitrogen in DOM, Aluwihare et al. [236] coupled mild acid hydrolysis with ${ }^{15} \mathrm{~N}$ NMR and revealed that more labile proteinaceous amide nitrogen is present in the surface layer, whereas more recalcitrant chitin- and peptidoglycan-derived amides accumulate in the deeper ocean.

Characterization of nitrogen in aquatic OM was also approached using SPIDER NMR [115], as discussed above. Unlike ${ }^{15} \mathrm{~N}$ NMR, which underrepresents nonprotonated $\mathrm{N}$ due to the use of ${ }^{1} \mathrm{H}^{-15} \mathrm{~N}$ cross-polarization, SPIDER NMR detects $\mathrm{N}$ in heterocycles as well as amide N. Using the SPIDER technique, Mao et al. [157] identified $\mathrm{sp}^{2}$-hybridized carbon resonating at $157 \mathrm{ppm}$ and bonded to at least two nitrogen atoms (associated with the unique peak at 140 ppm in the ${ }^{15} \mathrm{~N}$ CP/MAS spectrum) in a nitrogen-rich fulvic acid from Pony Lake in Antarctica. In a later study using optimized ${ }^{1} \mathrm{H}_{-}{ }^{15} \mathrm{~N}$ cross-polarization and ${ }^{15} \mathrm{~N}$ spectral editing by dipolar dephasing in conjunction with ${ }^{15} \mathrm{~N}$ NMR of model compounds, Fang et al. [116] proposed 
hydantoin derivatives as likely candidate structures that could account for the observed chemical shifts. Heterocycles were also found to constitute a significant fraction of the aromatic structures in POM [55].

\subsubsection{Solid-state ${ }^{31} P$ NMR applications}

Early studies applied ${ }^{31} \mathrm{P}$ solid-state NMR to high-molecular-weight DOM and POM to obtain the bulk distribution of different P compound classes throughout the world's oceans [239-243]. These studies found that the two major compound classes, phosphorus esters and phosphonates, occur in approximately constant proportions $(\sim 3: 1)$ regardless of water depth or distance from shore. The ${ }^{31} \mathrm{P}$ spectrum of POM from various sources contained a single peak due to phosphorus esters [240-242]. More recently, ${ }^{31} \mathrm{P}$ NMR analysis was applied to more representative DOM fractions isolated by reverse-osmosis coupled with electrodialysis from coastal and offshore environments [244]. The authors found a greater proportion of polyphosphates and phosphorus esters, and a lower proportion of phosphonates in these DOM samples than previously reported for high-molecular-weight DOM, suggesting that polyphosphates may be enriched in the low-molecular-weight, and phosphonates in the highmolecular-weight fraction [244].

\subsection{Organic matter in atmospheric particulate matter}

Atmospheric particulate matter is the microscopic solid or liquid matter in the atmosphere. Another commonly used term, aerosol, refers to the particulate and air mixture [245]. Atmospheric particulate matter contains organic matter and inorganic materials, and its sources can be either anthropogenic or natural. Its composition is closely correlated with environmental 
factors such as direct and indirect climate modifications, air pollution, and reduced visibility [246-248]. The organic matter in atmospheric particulate matter affects solar radiation through scattering and absorption, and analyzing its structure helps understanding of atmospheric chemistry and environmental effects [249]. Therefore, it is critical to study the structures of organic matter in atmospheric particulate matter.

Most published studies on the characterization of organic matter in atmospheric particulate matter have been conducted on the water-soluble fraction using ${ }^{1}$ H NMR [246], using solution NMR. Note that even 2D ${ }^{1} \mathrm{H}^{13} \mathrm{C}^{1}$ heteronuclear single quantum coherence (HSQC), heteronuclear multiple quantum coherence (HMQC), and heteronuclear multiple bond coherence (HMBC) can detect only protonated carbons. Thus, the structural features of atmospheric particulate organic matter obtained with these studies lack structural information on nonprotonated carbon. In addition, insoluble fractions should also be characterized. Therefore, solid-state NMR is essential for providing relatively complete structural information on atmospheric organic matter. Interested readers are strongly encouraged to consult two recent comprehensive reviews by Duarte and Duarte $[250,251]$ on the application of NMR spectroscopy in atmospheric organic matter. To the best of our knowledge, there are only a small number of studies using solid-state NMR to characterize atmospheric organic matter (see below). In almost all these, the simple ${ }^{13} \mathrm{C} C P / M A S$ technique has been used; advanced solidstate techniques have yet to be applied.

The ${ }^{13} \mathrm{C}$ CP/MAS spectra of water-soluble atmospheric organic matter extracted by XAD-8 during the summer and autumn seasons showed a dominance of alkyl signals between 0-50 ppm [252]. The summer sample contained smaller fractions of aromatics and aldehydes/ketones than that collected during the autumn. Duarte et al. then compared the 
structural features of water-soluble organic compounds from atmospheric aerosols to those of aquatic humic substances, and concluded that they had similar carbon functional groups although their relative proportions differed [253]. Sannigrahi et al. [254] characterized watersoluble organic carbon in urban atmospheric aerosol fine particles (PM2.5) by ${ }^{13} \mathrm{C} \mathrm{CP} / \mathrm{MAS}$ and found that it was primarily aliphatic, in addition to $\sim 10 \%$ carboxylic acids and $\sim 4 \%$ aromatics.

The macromolecular organic components of atmospheric particulate matter were also investigated using ${ }^{13} \mathrm{C}$ CP/MAS NMR [249]. These spectra indicated that alkyls were dominant in the atmospheric particulate matter, while the spectrum of its humic acid contained two bands of similar intensity attributed to alkyls and aromatics respectively, in addition to a small $\mathrm{COO} / \mathrm{N}-\mathrm{C}=\mathrm{O}$ band around $172 \mathrm{ppm}$. Zhao et al. [255] studied 12 dust samples from urban and suburban area of Guangzhou, China, using ${ }^{13} \mathrm{C}$ CP/MAS and other techniques. They separated the organic macromolecules in the dust into humic acids, kerogen and black carbon. ${ }^{13} \mathrm{C}$ CP/MAS spectra indicated that the major organic macromolecules were kerogen and black carbon [255]. Solum et al. [256, 257] studied structures and formation of aerosols and soot produced from model compounds, coal, and anthracene using ${ }^{13} \mathrm{C} \mathrm{CP} / \mathrm{MAS}$.

\subsection{Organic matter in carbonaceous meteorites}

The organic matter in primitive carbonaceous meteorites preserves a unique record of interstellar or nebular synthesis. In addition, detailed structure examination of the macromolecules in the carbonaceous chondrites can provide insight into the prebiotic chemical synthesis and subsequent alterations (e.g., aqueous alteration, thermal alteration) of organic compounds. 
Chondritic meteorites contain 1 to $2 \%$ of organic matter, most (70-90\%) of which is insoluble in water and solvents [258]. The less abundant, soluble fraction has been extensively investigated and its composition is relatively well known [259-261]. However, the structure of the dominant insoluble fraction has not been clearly resolved [260, 262-265]. These challenges have spurred the development of spectroscopic methods better suited for nondestructively analyzing the structure of solid, insoluble organic matter [266]. Among them, solid-state NMR is considered as the most effective means [266]. However, currently, the structural information on insoluble organic matter from meteorites is rather limited [258], and only a few attempts have been made to use solid-state NMR to decode its structure [266-270].

The pioneering work was performed by Cronin et al. [268], who investigated insoluble organic matter from three carbonaceous chondrites (Orgueil (C1), Murchison (CM2), and Allende (CV3)), using standard ${ }^{13} \mathrm{C} \mathrm{CP/MAS}$ as well as direct-polarization experiments. All spectra reported in this study were rather noisy probably due to high ash contents, and thus high levels of paramagnetic impurities. The authors concluded that these spectra showed only two major resonances, attributable to carbon in aliphatic and aromatic/olefinic structures. More than a decade later, Gardinier et al. [269] investigated the insoluble organic matter of the Orgueil and Murchison meteorites using ${ }^{13} \mathrm{C}$ CP/MAS NMR to obtain "quantitative" structural information. In this study, they claimed that eight different types of carbons were identified, and further structural information was obtained using deconvolution. Cody et al. [266] reinvestigated the insoluble organic matter in the Murchison meteorite using a purer sample obtained through improved preparation methods. They used a relatively wide range of solidstate NMR techniques: ${ }^{13} \mathrm{C}$ CP/MAS, ${ }^{13} \mathrm{C}$ DP/MAS, long-time dipolar dephasing, and singlepulse ${ }^{1} \mathrm{H}-\mathrm{NMR}$ at a high spinning frequency of $30 \mathrm{kHz}$. Although Cody et al. [266] made some 
progress in these studies, ambiguous assignments and component quantifications persisted. Later, they applied similar techniques to investigate four different carbonaceous chondrite meteorite groups and reported a progressive trend in chemical oxidation [267]. Using twodimensional magic angle turning (MAT) and other solid-state NMR techniques, Cody et al. [271] sought to establish the molecular relationship between chondritic and cometary organic solids. Their results suggested that chondritic insoluble organic matter and cometary refractory organic solids could be chemically related and derived from formaldehyde polymers [271]. Kebukawa et al. [272] further explored the possibility of the formation of organic solids in chondrites and comets through polymerization of interstellar formaldehyde. Yatuba et al. used ${ }^{13} \mathrm{C}$ solid-state NMR to study insoluble organic matter from Antarctic CM2 chondrites and the Murchison meteorite [64]. The spectra of the insoluble organic matter from the Murchison and Y-791198 meteorites showed two major peaks, attributed to aliphatics and aromatics. However, spectra from the other meteorites displayed only one major band, from aromatics. In this study, the atomic hydrogen-to-carbon ratio ('H:C') was used as a parameter for the thermal alteration event on the meteorite parent body. Due to the abundance of paramagnetic materials in carbonaceous meteorites, it is necessary to investigate how magnetic particles affected NMR spectra. Levin et al. [52] examined the effect of magnetic particles on NMR spectra of Murchison meteorite organic matter and a polymer-based model system. Organic matter from the Murchison meteorite showed pronounced spinning sidebands in the ${ }^{1} \mathrm{H}$ MAS NMR spectrum, indicating the presence of a combination of dipolar couplings of a given ${ }^{1} \mathrm{H}$ to magnetic particles and to other protons. Later, Yabuta et al. [273] also used ${ }^{13} \mathrm{C}$ solid-state NMR and other analytical methods to gain the insight into the chemical history of an ungrouped type 2 carbonaceous chondrite meteorite, Wisconsin Range (WIS) 91600. 
Recently, Sutter's Mill meteorite insoluble organic material was studied by solid-state NMR and the results were compared with those from other meteorites of diverse classifications [274], and the Tagish Lake meteorite was investigated using many different techniques, including solid-state NMR [275]. Moreover, ${ }^{15} \mathrm{~N}$ CP/MAS was also employed to investigate the $\mathrm{N}$ forms in Orgueil and Murchison meteorites [276]. ${ }^{15} \mathrm{~N}$ NMR spectra of insoluble organic matter from Orgueil indicated that nitrogen was present in pyrrole, indole, and carbazole moieties.

The authors have applied quantitative, selective and 2D NMR techniques to study the insoluble organic matter from the Murchison meteorite (Fig. 23). The DP/MAS spectrum (Fig. 23(a)) collected at a spinning frequency of $30 \mathrm{kHz}$ shows two dominant broad bands assigned to aliphatic (0-100 ppm, $\mathrm{sp}^{3}$-hybridized) and aromatic ( $\mathrm{sp}^{2}$-hybridized) carbons, the majority of which, aromatics in particular, are nonprotonated as shown by the DP/MAS spectrum with recoupled dipolar dephasing (Fig. 23(b)). In addition, the dipolar-dephased spectrum (Fig. 23(b)) shows a quaternary carbon peak at $\sim 35 \mathrm{ppm}$, which is characteristic of carbons in nanodiamond (Fig. 5(c)) and therefore suggests that most of $\mathrm{sp}^{3}$-hybridized carbons are attributed to those of nanodiamond. The quaternary carbon signal is remarkably underrepresented in the CP/TOSS spectrum (Fig. 23(c)), as is also the case in the CP/TOSS spectrum of nanodiamond (Fig. 5(c)). Spectral-editing techniques such as CSA-filtering, CHselection, and $\mathrm{CH}_{2}$-selection have been used to identify specific functional groups and make assignments reliably. For instance, the $\mathrm{CP}$ spectrum with a ${ }^{13} \mathrm{C}$ CSA filter selects only signals of $\mathrm{sp}^{3}$-hybridized carbons, and by comparing with the reference CP/TOSS spectrum (Fig. $23(\mathrm{c})$ ), it indicates that all the signals resonating above $100 \mathrm{ppm}$ are from $\mathrm{sp}^{2}$-hybridized carbons. The ${ }^{13} \mathrm{C}$ CSA filter and short $\mathrm{CP}$ selects signals of protonated $\mathrm{sp}^{3}$-hybridized carbons 
(Fig. 23(e)). ${ }^{13} \mathrm{C}$ CSA filter and dipolar dephasing retains signals of nonprotonated and mobile sp ${ }^{3}$-hybridized carbons, and its spectrum (Fig. 23(f)) shows major contributions from $\mathrm{CH}_{3}$ and underrepresented quaternary carbons. The $\mathrm{CH}$-only spectrum (Fig. 23(g)) shows signals of aromatic $\mathrm{C}-\mathrm{H}$ bands around $125 \mathrm{ppm}, \mathrm{OCH}$, and overlapping $\mathrm{NCH}(\sim 55 \mathrm{ppm})$ and $\mathrm{CCH}(\sim 40$ ppm). In addition, some $\mathrm{OCH}$ signals are obviously above baseline. The $\mathrm{CH}_{2}$-only spectrum (Fig. 23(h)) displays a strong $\mathrm{C}-\mathrm{CH}_{2}-\mathrm{C}$ band around $30 \mathrm{ppm}$. Overall these spectra demonstrate that CP-based techniques fail to provide a complete picture of the composition of insoluble organic matter in carbonaceous meteorites, and also that spectral-editing techniques are capable of identifying many more functional groups. The ${ }^{1} \mathrm{H}-{ }^{13} \mathrm{C} 2 \mathrm{D}$ HETCOR spectrum with dipolar dephasing (Fig. 23(i)) reveals connectivities between nonprotonated carbons and mobile $\mathrm{CH}_{3}$ and their surrounding protons. The ${ }^{1} \mathrm{H}$ slices shown in Figure 23(j) display the specific connectivities more clearly. For instance, the proton slice extracted at $17 \mathrm{ppm}\left(\mathrm{CH}_{3}\right)$ shows that $\mathrm{CH}_{3}$ is attached to aromatics, and the $\mathrm{C}=\mathrm{O}(200 \mathrm{ppm})$ is seen to be bonded to alkyl groups.

\subsection{Fossil fuels}

Solid-state NMR spectroscopy has been extensively used in nondestructive studies of fossil fuels including coal, oil shale and kerogen samples. The development of the CP/MAS technique greatly facilitated NMR applications to such complex organic matter types [65, 277286]. Excellent reviews of early applications of NMR techniques to solid fuels can be found elsewhere $[30,66]$. Recent work has also summarized structural studies of oil shale and kerogen using solid-state NMR [287], and has discussed, in a historical perspective, how solidstate NMR has been used for coal characterization [11]. This section is intended to summarize 
the various advanced NMR techniques that have been employed to elucidate structures of fossil fuels.

The spectra of solid fuels based on ${ }^{13} \mathrm{C} \mathrm{CP} / \mathrm{MAS}$ or quantitative DP methods enable the direct measurement of carbon aromaticity, and when combined with dipolar dephasing techniques also the relative amounts of nonprotonated and protonated carbon. However, these spectra are broad due to the presence of a large number of resonances, and no clean divisions exist between bands due to different functional groups. This has spurred the development of more advanced solid-state NMR methods, which were primarily applied to coals between the late 1980s and 2000. For example, Zilm and Webb [164] demonstrated several new 2D NMR methods for fossil fuels, including heteronuclear shift correlation, dipolar resolved chemical shift, and zero-field NMR. Zilm's group further developed experiments for spectral editing of ${ }^{13} \mathrm{C} \mathrm{CP} / \mathrm{MAS}$ spectra by using polarization, polarization inversion, and spin depolarization, generating a $\mathrm{CH}_{2}$-only subspectrum, a $\mathrm{CH}$-only subspectrum, and a spectrum with $\mathrm{CH}_{3}$ and nonprotonated $\mathrm{C}[80,82]$. $\mathrm{Hu}$ et al. [288] utilized these pulse sequences with some modifications to separate nonprotonated $\mathrm{C}$ and $\mathrm{CH}_{3}$ carbon peaks unequivocally, and applied isotropic-anisotropic chemical shift correlation to derive structural information in the aromatic region of coal. In addition, discrimination of two kinds of nonprotonated carbons (condensed and alkyl substituted aromatic carbons), which are not resolved using dipolar dephasing, was also attempted by exploiting the differences between their total shielding band shapes [289].

More recently, Mao and Schmidt-Rohr developed and applied a systematic approach to characterize fossil solids such as kerogen and coal, which includes identification and quantification of specific functional groups, their connectivities or proximities, heterogeneities and domains [178, 287, 290-292]. In addition to spectral editing of $\mathrm{CH}_{-}$-only, $\mathrm{CH}_{2}$-only, and 
$\mathrm{CH}_{3}$ and nonprotonated carbons, a CSA filtering technique can be used to selectively suppress the signal of $\mathrm{sp}^{2}$ aromatic carbons in the $90-120 \mathrm{ppm}$ region and retain the signal from $\mathrm{sp}^{3}$ carbons. Moreover, the ${ }^{1} \mathrm{H}-{ }^{13} \mathrm{C}$ long-range recoupled dipolar dephasing technique can select interior aromatic carbons in fused aromatic rings whose signals remain at the $>50 \%$ level after $0.9 \mathrm{~ms}$ of recoupled dipolar dephasing, and offers unique insight into aromatic cluster size in coal or kerogen structures.

Note that NMR characterization of fossil fuels has almost exclusively been performed at low magnetic fields, at $9.4 \mathrm{~T}$ (corresponding to a ${ }^{1} \mathrm{H}$ resonance frequency of $400 \mathrm{MHz}$ ) or lower and at spinning rates $\leq 20 \mathrm{kHz}$, because of several potential drawbacks associated with using high fields and high MAS rates [93]. Recent work by Pruski's group [293, 294], however, demonstrated that high magnetic fields and fast MAS utilized in coal studies offered some advantages (the possibility of using ${ }^{1} \mathrm{H}$ detection of ${ }^{13} \mathrm{C}$ in $2 \mathrm{D}$ heteronuclear correlation schemes and simplified pulse sequences), with only slightly lower sensitivity. However, it

remains difficult to detect interior aromatic carbons or obtain quantitative ${ }^{13} \mathrm{C}$ spectra under these conditions.

Advanced solid-state NMR characterization clearly offers more detailed structural information on fossil fuels with complex molecular structures than do traditional ${ }^{13} \mathrm{C} \mathrm{CP} / \mathrm{MAS}$ techniques. Unfortunately, however, advanced solid-state NMR techniques with newly implemented pulse programs or high magnetic fields are not readily accessible to many researchers in this field, and such applications to the study of fossil fuels have been primarily limited to a few research groups.

\section{Structural models based on NMR-derived structural information}


In order to advance our understanding of NOM structure, NMR should provide a comprehensive analysis of the chemical composition of the material under investigation. The traditional integration table of functional group composition, in terms of fairly generic structural units, is based on often ambiguous assignments of overlapping peaks and may leave out possibly important components, such as nonprotonated carbons. In addition, many published tables of functional group abundances are derived primarily from ${ }^{13} \mathrm{C} \mathrm{CP} / \mathrm{MAS}$ or distorted ${ }^{13} \mathrm{C}$ DP/MAS spectra and are therefore not quantitative.

Through quantitative spectroscopy, spectral editing and 2D NMR, more structural information on specific functional groups, their connectivities and specific domains is obtained. Structural models proposed in the literature can be ruled out if their predicted spectra disagree significantly with the experimental data [48, 63, 159, 295, 296]. In favorable cases, if the spectra are relatively simple, a realistic structural model for the NOM under study can be proposed [48, 63, 159, 295, 296]. For example, the high-molecular-weight hydrophobic fraction of the biosolids-derived biocolloidal organic matter was investigated using quantitative ${ }^{13} \mathrm{C}$, CH spectral-editing, dipolar-dephasing, ${ }^{1} \mathrm{H},{ }^{15} \mathrm{~N}, 2 \mathrm{D}$ HETCOR, and 2D SUPER NMR, as well as ${ }^{1} \mathrm{H}$ chemical shift filtering with spin diffusion, in combination with elemental analysis [295]. It was found to consist predominantly of $\mathrm{N}$-acetylated polysaccharides, which are possibly derived from bacterial peptidoglycans [295]. A structural model was also proposed for char residues in both Terra Preta soils (fertile anthropogenic dark earths in Amazonia that were enriched with char $>800$ years ago) and a Mollisol soil from Iowa, based on quantitative ${ }^{13} \mathrm{C}$ NMR, ${ }^{1} \mathrm{H}_{-}{ }^{13} \mathrm{C}$ long-range dipolar dephasing as well as ${ }^{1} \mathrm{H}_{-}{ }^{13} \mathrm{C}$ 2D HETCOR [165]. This was possible because the spectra were relatively simple, being dominated by a strong monomodal band of mostly nonprotonated aromatic carbons near $130 \mathrm{ppm}$ and a somewhat less intense 
COO peak at $170 \mathrm{ppm}$. The proposed model contains approximately 6 fused aromatic rings extensively substituted by $\mathrm{COO}$ groups. The $\mathrm{COO}$ groups can significantly increase the cation exchange capacity of soils and thus promote the retention of plant nutrients. A model of a naturally occurring asphaltite bitumen, gilsonite, was also established based on advanced solidstate NMR techniques, solution NMR, and electrospray ionization Fourier transform ion cyclotron resonance mass spectrometry [159]. Gilsonite was proposed to comprise a mixture of many pyrrolic and a few fused aromatic rings highly substituted with, and connected by, mobile aliphatic chains.

\section{Summary and outlook}

Advanced solid-state NMR techniques have great potential to elucidate NOM structure at the molecular level, whose significance for NOM cycling in biogeochemical environments can then be assessed. Such a systematic approach enables generation of reliable and quantitative structural information, identification of specific functional groups using spectral-editing techniques, examination of proximities using 2D HETCOR, and investigations of domains and heterogeneities via spin diffusion techniques.

In order to obtain a reliable and quantitative ${ }^{13} \mathrm{C}$ solid-state NMR spectrum, DP/MAS or multiCP/MAS, but not simple CP/MAS, at a sufficiently high spinning frequency is highly recommended. A quantitative ${ }^{13} \mathrm{C}$ DP/MAS spectrum can be acquired following these guidelines: (1) use a rotation-synchronized Hahn echo to refocus magnetization before detection to avoid baseline distortions; (2) spin fast enough that aromatic-carbon sidebands are at $<0$ ppm (i.e. with a MAS frequency of $\omega_{\mathrm{r}} \geq 135 \mathrm{ppm}$ ); (3) apply strong, on-resonance

TPPM ${ }^{1} \mathrm{H}$ decoupling $\left(\gamma \mathrm{B}_{1} \geq 5 \omega_{\mathrm{r}}\right)$; (4) use a sufficiently long recycle delay $\left(\geq 4{ }^{13} \mathrm{C} T_{1}\right)$. 
MultiCP should be used for relatively hydrogen-rich and clean materials with long relaxation times, such as plant matter. The advanced spectral-editing techniques discussed in detail in this review include examples that select $\mathrm{CH}$ and $\mathrm{CH}_{2}$ groups, SPIDER for detecting carbons directly bonded to nitrogen, a ${ }^{13} \mathrm{C}$ CSA filter to separate $\mathrm{sp}^{2}$-hybridized from $\mathrm{sp}^{3}$-hybridized carbons, and recoupled ${ }^{1} \mathrm{H}-{ }^{13} \mathrm{C}$ long-range dipolar dephasing to select fused-ring aromatic signals. Combinations of different spectral-editing techniques allow for the identification of at least 27 different functional groups, in contrast to less than 10 previously reported. ${ }^{1} \mathrm{H}^{13} \mathrm{C} 2 \mathrm{D}$ HETCOR and 2D HETCOR combined with spectral-editing techniques such as dipolar dephasing reveal connectivities and proximities between different functional groups; for instance, they can provide information on the environments of $\mathrm{COO}$ groups and nonprotonated aromatic carbons in NOM. ${ }^{1} \mathrm{H}$ spin diffusion provides information on heterogeneities and domains of NOM. Appropriate techniques for investigating heterogeneities and domains include the Goldman-Shen spin diffusion experiment, ${ }^{1} \mathrm{H}$ inversion recovery, ${ }^{1} \mathrm{H}-{ }^{1} \mathrm{H}$ CRAMPS 2D exchange NMR, ${ }^{1} \mathrm{H}-{ }^{13} \mathrm{C} 2 \mathrm{D}$ HETCOR with spin diffusion, and ${ }^{1} \mathrm{H}-{ }^{13} \mathrm{C}$ recoupled long-range dipolar dephasing. The selection of appropriate techniques for studying domains and heterogeneities in particular cases is determined by the characteristics of NOM and also the available NMR hardware. If the spectra are sufficiently simple, the comprehensive structural information derived from advanced NMR techniques can be used to build reasonable structural models of NOM.

NMR has been extensively applied in the field of organic geochemistry: a search using the keywords "organic matter" and "NMR" leads to thousands of publications. However, a close examination reveals that the primary technique used has been simple ${ }^{13} \mathrm{C}$ or ${ }^{15} \mathrm{~N}$ CP/MAS. While advanced solid-state NMR techniques are extensively used in biomolecular NMR, they 
have so far been severely underexploited in the organic geochemistry field, mainly due to a lack of expertise and appropriate techniques as well as instruments and spectroscopists dedicated to organic geochemistry. Indeed, the setup of sophisticated NMR techniques requires specialized knowledge. NMR spectroscopists dedicated to pure-chemistry and biomolecular NMR are often not familiar with the appropriate conditions for acquisition and data processing of high-quality spectra from NOM samples. Thus, the gap between organic geochemistry and advanced solid-state NMR techniques needs to be bridged by education and special training. Although the applications of systematic advanced NMR techniques for NOM reviewed here collectively represent a significant step forward, the primary focus so far has been on ${ }^{13} \mathrm{C}$ techniques. Advanced ${ }^{15} \mathrm{~N}$ and ${ }^{31} \mathrm{P}$ solid-state NMR techniques, which would be valuable for investigating the important nitrogen and phosphorus cycles in nature, remain underexplored. Similarly to ${ }^{13} \mathrm{C}$ techniques, the development and application of advanced ${ }^{15} \mathrm{~N}$ and ${ }^{31} \mathrm{P}$ solidstate NMR techniques will have a far-reaching impact for the study of NOM. Furthermore, the interactions of NOM with other matrices such as organic contaminants and clays are important environmental topics for resolving issues of groundwater contamination, soil nutrient cycling, and carbon sequestration in soil.

\section{Acknowledgments}

Financial support by the NSF (EAR-1226323, EAR-0843996 and CBET-0853950), Petroleum Research Fund Type G grant (46373-G2), and the Thomas F. Jeffress and Kate Miller Jeffress Memorial Trust is gratefully acknowledged. 


\section{Figure Captions:}

Figure 1: Typical DP/MAS ${ }^{13} \mathrm{C}$ NMR spectra of NOM from various sources. (a) Peat from the International Humic Substances Society (IHSS), (b) insoluble organic matter from the Murchison meteorite, (c) rice soil humic acid from the International Rice Research Institute, (d) bituminous coal from Indiana, (e) dissolved organic matter from the Georgia coast, (f) aquatic fulvic acid from the Pony Lake in the Antarctica, (g) aquatic humic acid from the Saguenay Fjord, (h) humic acid from the sediment in the St. Lawrence Estuary, (i) kerogen 554-1 from the New Albany Shale, and (j) milled wood lignin from Loblolly pine (Pinus taeda). Spinning frequency: $v_{\mathrm{r}}=14 \mathrm{kHz}$. All these spectra are comprised largely of broad bands. Reproduced with permission from Refs. [55, 156, 157, 178, 218, 297].

Figure 2: ${ }^{13} \mathrm{C}$ NMR spectra of a mixture of three ${ }^{13} \mathrm{C}$-labelled amino acids: ${ }^{13} \mathrm{CH}_{3}$-labelled alanine, ${ }^{13} \mathrm{CH}_{2}$-labelled glycine, and ${ }^{13} \mathrm{COO}$-labelled leucine, used for setting up the timing of a Hahn echo so as to produce an optimized baseline. (a) Hahn-echo pulse sequence. (b) Perfect length of the second $\tau$ period, after the $\pi$-pulse and before detection, showing a flat, ideal baseline; (c) the duration of the second $\tau$ period is set $5 \mu$ s too short and thus the magnetization is detected $5 \mu$ s too early; the baseline beyond the peak regions bends down, below a flat baseline; and (d) the second $\tau$ period is set $5 \mu$ s too long and the magnetization is detected $5 \mu \mathrm{s}$ too late; the baseline beyond the peak regions bends up, above a flat baseline.

Figure 3: Comparison of DP/MAS ${ }^{13} \mathrm{C}$ NMR spectra of IHSS peat acquired using different timings and processing. Panel (a) shows a reliable, quantitative spectrum resulting from the use of an echo with correctly optimized timing. Panels (b)-(e) show spectra obtained when the 
second part of the echo is set (b) $5 \mu$ s too early, (c) $15 \mu$ s too early, (d) $5 \mu$ s too late, and (e) 15 $\mu$ s too late; Panels (f) and (g) show spectra obtained without using an echo, either without (f) or with (g) a first-order phase correction. The aliphatic carbon fraction, based on the relative integral of the 0-90 ppm region, is given for each spectrum. It is obvious that all the spectra shown in panels (b) through (g) are distorted relative to that shown in (a). The spectra acquired without using an echo are the most seriously distorted. All spectra were collected at $14 \mathrm{kHz}$ MAS using a recycle delay of $20 \mathrm{~s}$ and 1024 scans.

Figure 4: The effects of spinning rate as well as ${ }^{1} \mathrm{H}$ decoupling strength, decoupling method and resonance position on ${ }^{13} \mathrm{C}$ NMR spectra demonstrated using crystalline cholesterol acetate. Spectra in the left-hand column were acquired using a spinning frequency of $6.5 \mathrm{kHz}$ and those in the right column using $14 \mathrm{kHz}$. Spectra on the right are vertically scaled by a factor of 5 . Panels (a)-(h) show results for CW decoupling and panels (i)-(p) for TPPM decoupling; (a) and (b), CW decoupling at $48 \mathrm{kHz}$; (c) and (d), CW decoupling at $54 \mathrm{kHz}$; (e) and (f), CW decoupling at $61 \mathrm{kHz}$; (g) and (h), CW decoupling at $54 \mathrm{kHz}$ but off-resonance by $2.8 \mathrm{kHz}$; (i) and (j), TPPM decoupling at $48 \mathrm{kHz}$; (k) and (1), TPPM decoupling at $54 \mathrm{kHz}$; (m) and (n), TPPM decoupling at $61 \mathrm{kHz}$; and (o) and (p), TPPM decoupling at $54 \mathrm{kHz}$ but off-resonance by $2.8 \mathrm{kHz}$. All spectra were collected with cross polarization and a recycle delay of $4 \mathrm{~s}$, and typically 128 scans.

Figure 5: Several examples demonstrating differences between CP and DP spectra of the same samples. (a) A Mollisol humic acid with high aromaticity; (b) a humic acid sample extracted 
from sinking particles collected in the St. Lawrence Estuary; (c) nanodiamond with 5-nm diameter grains. Reproduced with permission from Ref. [55].

Figure 6: Principle of the $\mathrm{CP} / \mathrm{T}_{1}$ method for measuring ${ }^{13} \mathrm{C} \mathrm{T}_{1}$ signal decay. (a), (b), and (c) depict how magnetization evolves during a $+z$-filter, and (d), (e), and (f) display how magnetization evolves during a $-z$-filter.

Figure 7: $\mathrm{T}_{1 \rho}{ }^{\mathrm{H}}$ measurements on a sample of IHSS peat humic acid, using contact times of (a) $0.1 \mathrm{~ms}$, (b) $1 \mathrm{~ms}$, (c) $2 \mathrm{~ms}$, (d) $5 \mathrm{~ms}$, and (e) $10 \mathrm{~ms}$. Note that at a contact time of $10 \mathrm{~ms}$, almost no signals remain, making it impossible to obtain a quantitative spectrum on this sample using ramped-CP (given the requirement of a 10-ms contact time for quantitative spectra [59]).

Figure 8: Identification of ${ }^{13} \mathrm{C}$ NMR signals of specific functional groups in a fulvic acid sample from the Pony Lake in the Antarctica by spectral editing. (a) Quantitative ${ }^{13} \mathrm{C}$ NMR spectrum obtained by DP/MAS with 200-s recycle delay, at 14-kHz MAS. (b) ${ }^{13} \mathrm{C} \mathrm{CP/MAS}$ NMR spectrum, used as a reference for other spectral-edited spectra. (c) Same as (b) but with $40-\mu$ s dipolar dephasing to select signals of nonprotonated and mobile segments. Note the alkyl region of the spectrum is scaled vertically by a factor of three and stacked above it. (d) Selection of $\mathrm{sp}^{3}$-hybridized carbon signals by a ${ }^{13} \mathrm{C} \mathrm{CSA}$ filter, which in particular identifies OCO $\mathrm{sp}^{3}$-hybridized carbons, near $100 \mathrm{ppm}$. (e) Selection of immobile $\mathrm{sp}^{3}$-hybridized $\mathrm{CH}_{2}$ and

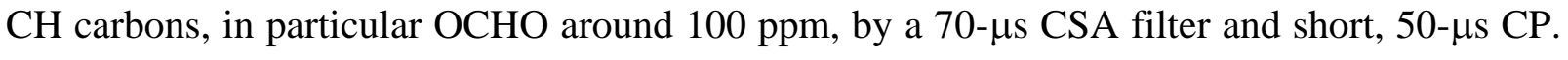
(f) Selection of nonprotonated or mobile $\mathrm{sp}^{3}$-hybridized carbon signals using a 70 - $\mu$ s CSA filter and 40- $\mu$ s dipolar dephasing, which in particular identifies $\mathrm{OC}_{\mathrm{q}} \mathrm{O}$ carbons near $110 \mathrm{ppm}$. 
(g) Selection of signals of $\mathrm{CH}_{3}$ and mobile $\mathrm{CH}_{2}$ groups in a DP/MAS experiment with 1.5-s recycle delay at 14-kHz MAS. (h) Combination of a ${ }^{13} \mathrm{C}_{1}$ filter and dipolar dephasing selects nonprotonated carbons and reduces signals from mobile groups such as $\mathrm{CH}_{3}$. (i) Selection of immobile $\mathrm{CH}$ and $\mathrm{CH}_{2}$ signals using short $\mathrm{CP}$ and dipolar-dephasing difference. (j) Signals of immobile $\mathrm{CH}_{2}$ groups, selected based on three-spin-coherence, at 5.8-kHz MAS. $\mathrm{CH}_{2}$ and $\mathrm{OCH}_{2}$ bands are clearly seen. (k) $\mathrm{CH}$-only spectrum, obtained by dipolar DEPT at a 4-kHz spinning frequency. $\mathrm{OCH}$ and $\mathrm{NCH}$ bands are clearly observed at 70 and $55 \mathrm{ppm}$, respectively. Downfield and at lower intensity levels, OCHO and aromatic $\mathrm{CH}$ bands are detected. (1) Identification of signals of nitrogen-bonded carbons by ${ }^{13} \mathrm{C}\left\{{ }^{14} \mathrm{~N}\right\}$ SPIDER NMR. (m) SPIDER NMR after 40- $\mu$ s of dipolar dephasing, which selects nonprotonated carbons bonded to nitrogen. Reproduced with permission from Ref. [157].

Figure 9. NMR spectra of a humic acid extracted from sediment in the Saguenay Fjord (Canada). (a) CP/TOSS, showing obvious carbonyl bands around 200 ppm; (b) Dipolardephased spectrum, with almost all the carbonyl signal retained; and (c) Protonated-carbononly spectrum, without carbonyl signal. This indicates that all the carbonyl groups detected in this humic acid are nonprotonated ketones. Reproduced with permission from Ref. [232].

Figure 10. 2D ${ }^{1} \mathrm{H}-{ }^{13} \mathrm{C}$ HETCOR spectra of (a) a peat humic acid and (b) the corresponding insoluble humin, showing identification of $\mathrm{COOH}$ in humic acid, but not in humin, based simply on the distinctive chemical shift of $\mathrm{COOH}$ protons. Reproduced with permission from Refs. [24, 175]. 
Figure 11. Sheared 2D SUPER ${ }^{13} \mathrm{C}$ spectrum of the humin of IHSS peat from Florida, at $5 \mathrm{kHz}$ MAS.

Figure 12. Examples of spectra of functional groups based on the ${ }^{13} \mathrm{C}$ chemical-shift anisotropy. Top row: $\mathrm{COOH}$ and $\mathrm{COO}^{-}$groups, bottom row: $\mathrm{COOC}$ esters, with the peak characteristically on the right-hand side of the powder spectrum. Reproduced with permission from Refs. [137, 295].

Figure 13. ${ }^{13} \mathrm{C}\left\{{ }^{14} \mathrm{~N}\right\}$ NMR spectrum of carbons directly bonded to nitrogen in a rice soil organic matter sample, obtained by the SPIDER technique. Top (thin-lined): reference spectrum showing all carbons. Bottom (thick-lined): spectrum of carbons directly bonded to nitrogen. Bands of $\mathrm{N}-\mathrm{C}=\mathrm{O}, \mathrm{NCH}$, and $\mathrm{N}$ bonded to aromatic $\mathrm{C}$ are clearly recognized. Reproduced with permission from Ref. [156].

Figure 14. ${ }^{13} \mathrm{C}$ NMR spectra of gilsonite bitumen. (a) The CP/TOSS spectrum shows qualitative structural information. (b) $\mathrm{CH}_{2}$ selection clearly displays an exomethylene resonance around $125 \mathrm{ppm}$. Reproduced with permission from Ref. [159].

Figure 15. Approximate chemical-shift positions of 36 chemical moieties relevant in NOM that can be identified using advanced ${ }^{13} \mathrm{C}$ NMR techniques reported by the authors. At the top, traditional chemical-shift range assignments are shown; overlap is indicated by dashed lines. In the structures shown, bonded $\mathrm{H}$ (in red) enables spectral editing; quaternary carbons easily identified and quantified by dipolar dephasing are marked by dashed red circles. Solid green 
circles marks alkyl carbons whose resonances can be separated from overlapping aromatic bands by chemical-shift anisotropy filtering. Bonded $\mathrm{N}$ can be identified through its chemical shift effects or by ${ }^{13} \mathrm{C}\left\{{ }^{14} \mathrm{~N}\right\}$ SPIDER NMR. Bonded $\mathrm{O}$ typically increases the chemical shift by $30 \mathrm{ppm}$. Information on the 'R' groups can often be obtained by 2D NMR methods.

Figure 16. ${ }^{1} \mathrm{H}$ spin diffusion with ${ }^{13} \mathrm{C}$ NMR detection after cross polarization, used for identifying the proximity of the mobile acyl chains to the other components in this aquatic humic acid from the St. Lawrence Estuary. A series of spectra with increasing spin diffusion time is shown. Magnetization of protons on the mobile acyl chains with carbon resonances around $30 \mathrm{ppm}$ has been selected by a ${ }^{1} \mathrm{H} \mathrm{T}_{2}$ filter (Goldman-Shen experiment), and therefore their signals dominate the spectra at short spin-diffusion times. After longer spin-diffusion times (> $1 \mathrm{~ms}$ ), the $\mathrm{C}=\mathrm{O}, \mathrm{OCH}$, and $\mathrm{NCH}$ signals all reappear, while the acyl-chain signals decrease in intensities. After long times, there are indications that the phenolic and aromatic components, which are more difficult to detect due to their low signal intensities, have also received magnetization. Reproduced with permission from Ref. [55].

Figure 17. 2D HETCOR spectra with ${ }^{1} \mathrm{H}$ spin diffusion, for a peat humic acid sample. (a) Spectrum acquired with a very short mixing time of $10 \mu \mathrm{s}$; the carbon slice at the aromatic proton chemical shift shows that magnetization is still mainly confined to the aromatic region in the carbon dimension. (b) Spectrum acquired with a mixing time of $200 \mu$ s; the carbon slice at the aromatic proton chemical shift shows that now spin diffusion has occurred transferring magnetization to the $\mathrm{OCH}$ region. (c) A series of carbon slices extracted at the aromatic proton chemical shift from 2D HETCOR spectra, showing progressive spin diffusion from aromatics 
to $\mathrm{OCH}$ as a function of mixing time, which can provide information about heterogeneity of this humic acid. Reproduced with permission from Ref. [24].

Figure 18. A series of CRAMPS $2 \mathrm{D}$ exchange ${ }^{1} \mathrm{H}$ NMR spectra of whole soil organic matter from a rice soil in the Philippines with mixing times of (a) $3 \mu \mathrm{s}$, (b) $300 \mu \mathrm{s}$, (c) $1 \mathrm{~ms}$, and (d) $10 \mathrm{~ms}$.

Figure 19. Protonated-carbon-only spectra of (a) lignin, (b) triple-cropped soil MHA, and (c) dryland soil MHA.

Figure 20. (a) Structure of a typical lignin unit, (b) CP/TOSS ${ }^{13} \mathrm{C}$ spectrum of lignin, (c) 2D HETCOR spectrum of lignin, and (d) 2D HETCOR spectrum combined with $\mathrm{OH}$ and $\mathrm{NH}$ suppression for a peat HA sample. Note that the 2D HETCOR spectra of both lignin and the peat HA clearly show the aromatic-methoxyl cross-peak pattern characteristic of aromatic C$\mathrm{OCH}_{3}$ moieties. Reproduced with permission from Refs. [24, 112].

Figure 21. (a) A series of DP/TOSS ${ }^{13} \mathrm{C}$ spectra of IHSS Leonardite humic acid after recoupled dipolar dephasing for the indicated durations $t_{\mathrm{dd}}$, showing selection of fused ring C. (b) Longrange dipolar dephasing curves $S / S_{0}$ for aromatics of char, carbonate in char, IHSS peat HA, IHSS Leonardite HA, and lignin, from DP/TOSS spectra. Reproduced with permission from Ref. [114]. 
Figure 22. Identification of mobile aliphatic chains using ${ }^{13} \mathrm{C}$ NMR. (a) CP/TOSS spectrum showing all the semi-quantitative structural information; (b) DP/MAS ${ }^{13} \mathrm{C}$ spectrum recorded with a 1.5-s recycle delay at a spinning frequency of $13 \mathrm{kHz}$; (c) expansion of the aliphatic region of the spectrum in (b) including detailed assignments; (d) ${ }^{1} \mathrm{H}$ NMR spectrum of the mobile aliphatic chains; and (e) typical structure of a mobile aliphatic acyl chain. Reproduced with permission from Ref. [55].

Figure 23: Quantitative, spectral-editing, and 2D NMR spectra of insoluble organic matter from the Murchison meteorite. (a) DP spectrum measured at $30 \mathrm{kHz}$ MAS. (b) DP/MAS at 30 $\mathrm{kHz}$ with dipolar dephasing, giving quantitative structural information of nonprotonated $\mathrm{C}$ plus $\mathrm{CH}_{3}$. (c)-(h). Spectral editing for identification of specific carbon functional groups. (c) Unselective CP/TOSS spectrum for reference. (d) Selection of $\mathrm{sp}^{3}$-hybridized carbon signals by a ${ }^{13} \mathrm{C}$ chemical shift anisotropy filter. CSA filter time: $35 \mu$ s. (e) Selection of protonated $\mathrm{sp}^{3}$ hybridized carbon signals by a ${ }^{13} \mathrm{C}$ chemical shift anisotropy filter. CSA filter time: $35 \mu$ s. CP: $50 \mu \mathrm{s}$. (f) Selection of nonprotonated and mobile $\mathrm{sp}^{3}$-hybridized carbon signals by a ${ }^{13} \mathrm{C}$ chemical shift anisotropy filter combined with dipolar dephasing. CSA filter time: $35 \mu$ s. dipolar-dephasing time: $40 \mu \mathrm{s}$. (g) Selection of CH signals by dipolar DEPT. Spinning speed: 4 kHz. (h) Signals of immobile $\mathrm{CH}_{2}$ groups selected based on three-spin-coherence, at a spinning speed of $5.787 \mathrm{kHz}$. (h) Spectrum of 2D HETCOR with dipolar dephasing. (i) ${ }^{1} \mathrm{H}$ slices extracted at: $17,35,50,60,80,130,173,200 \mathrm{ppm}$. These data have not been published and the Murchison meteorite insoluble organic matter was provided by Dr. Yongsong Huang at Brown University. 

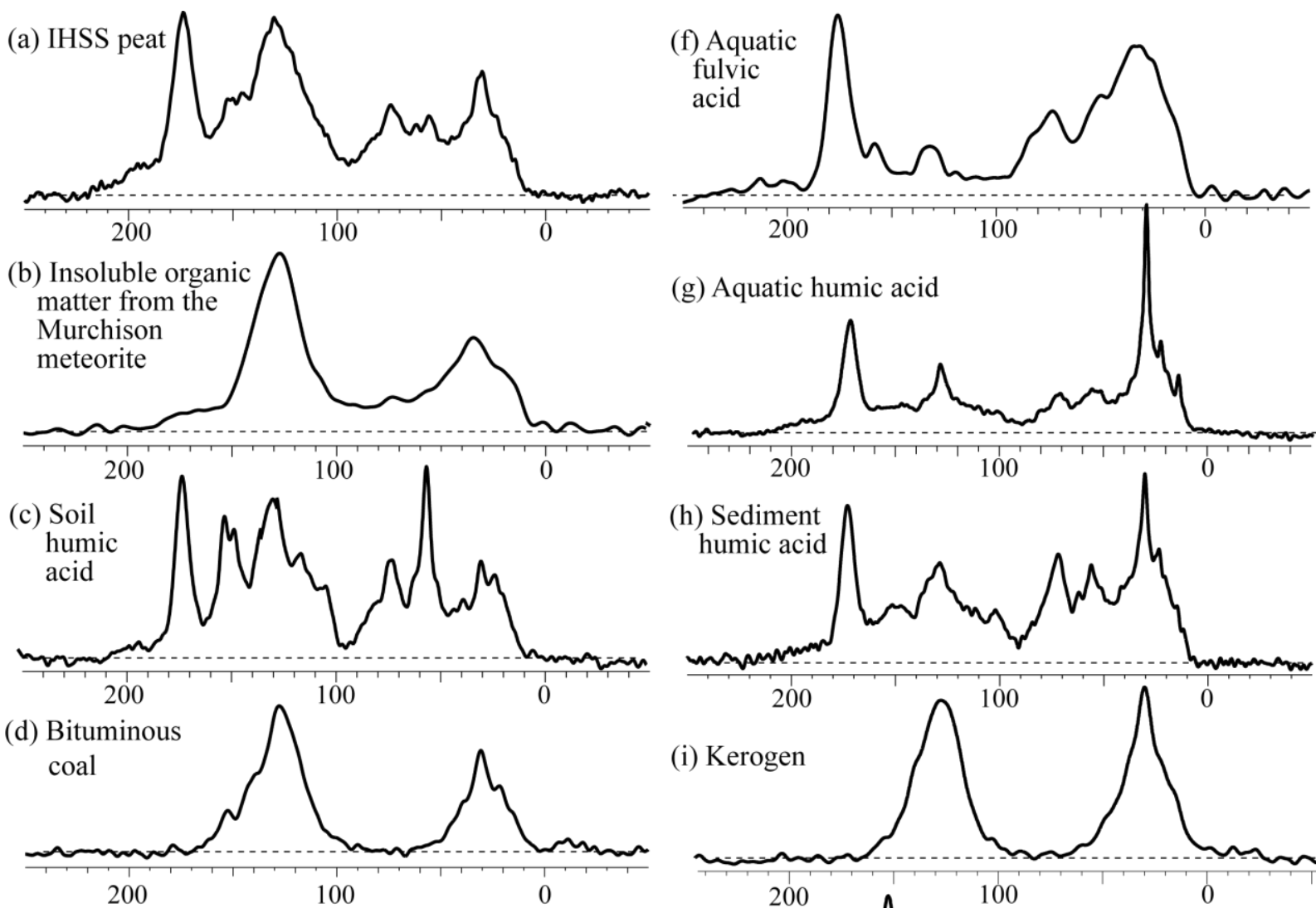

(g) Aquatic humic acid
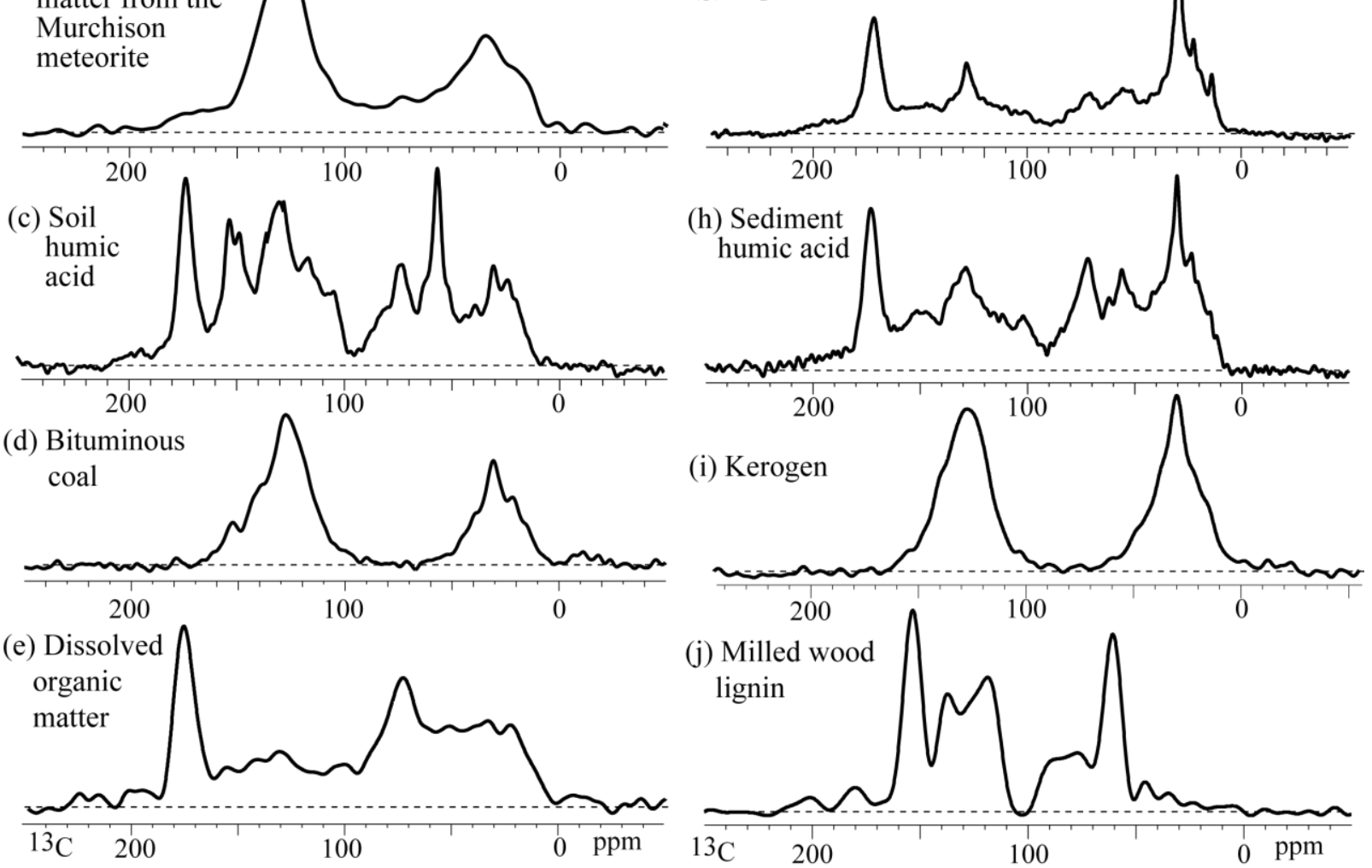

Figure 1 Mao et al. 


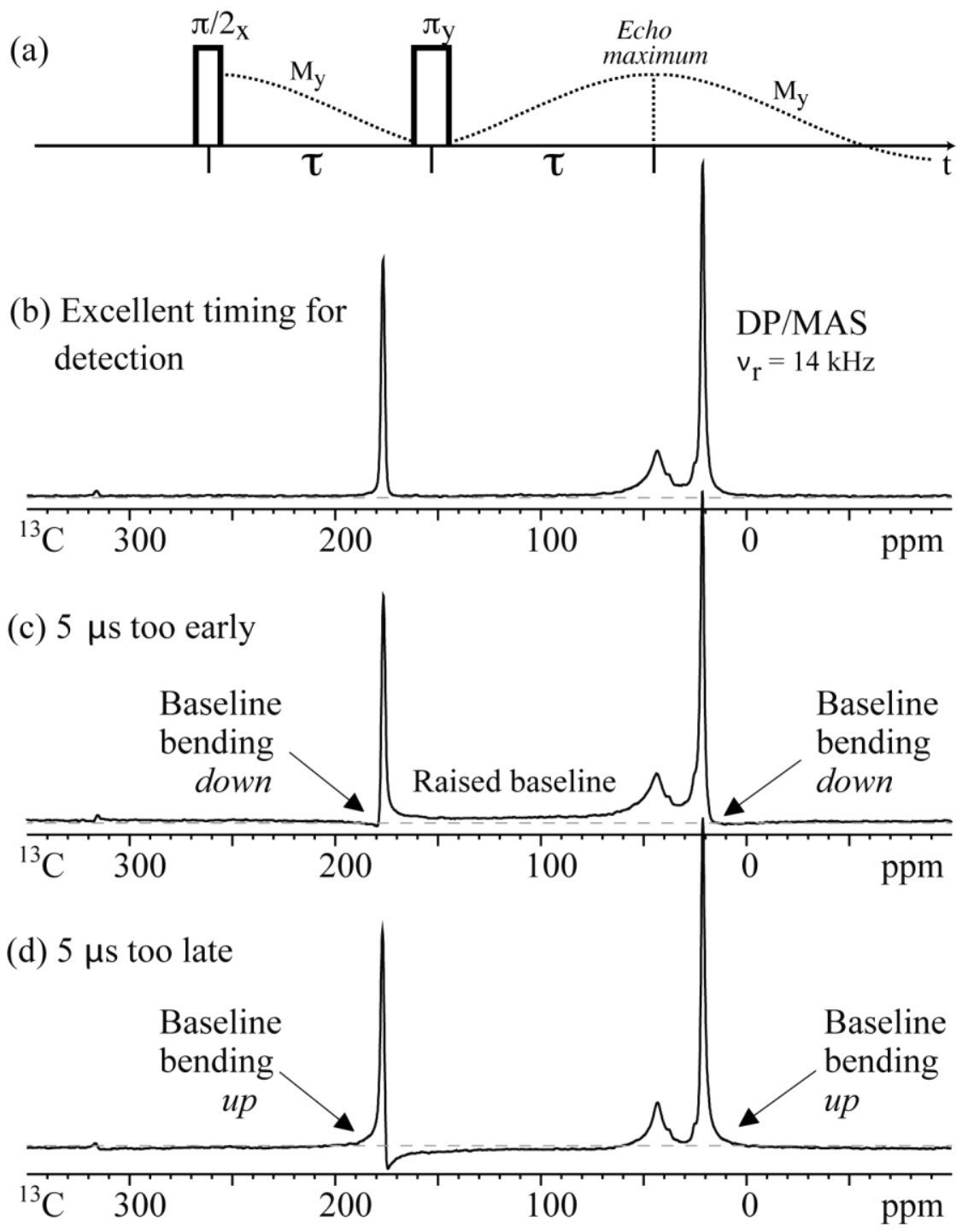

Figure 2. Mao et al. 


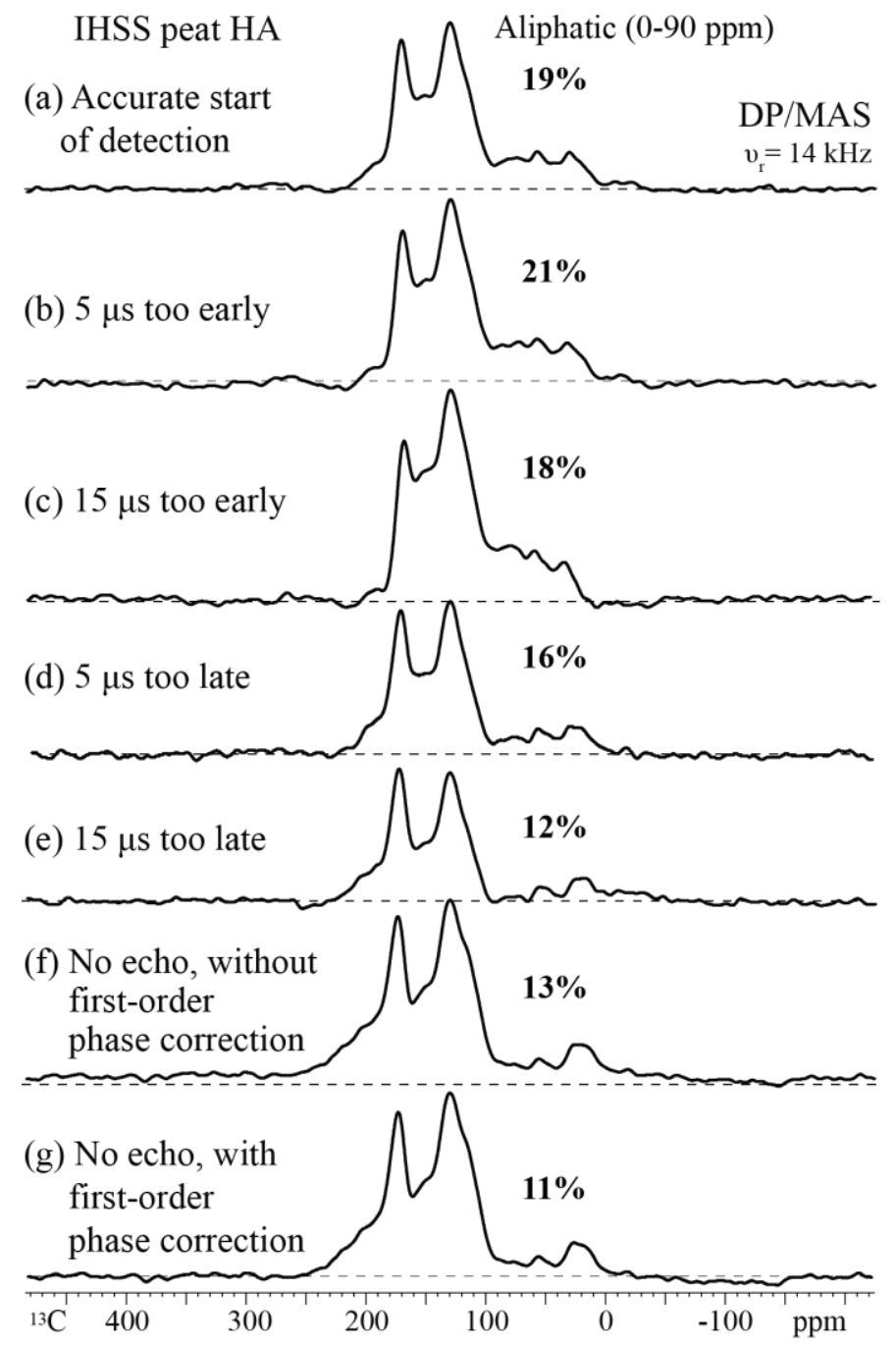

Figure 3. Mao et al. 


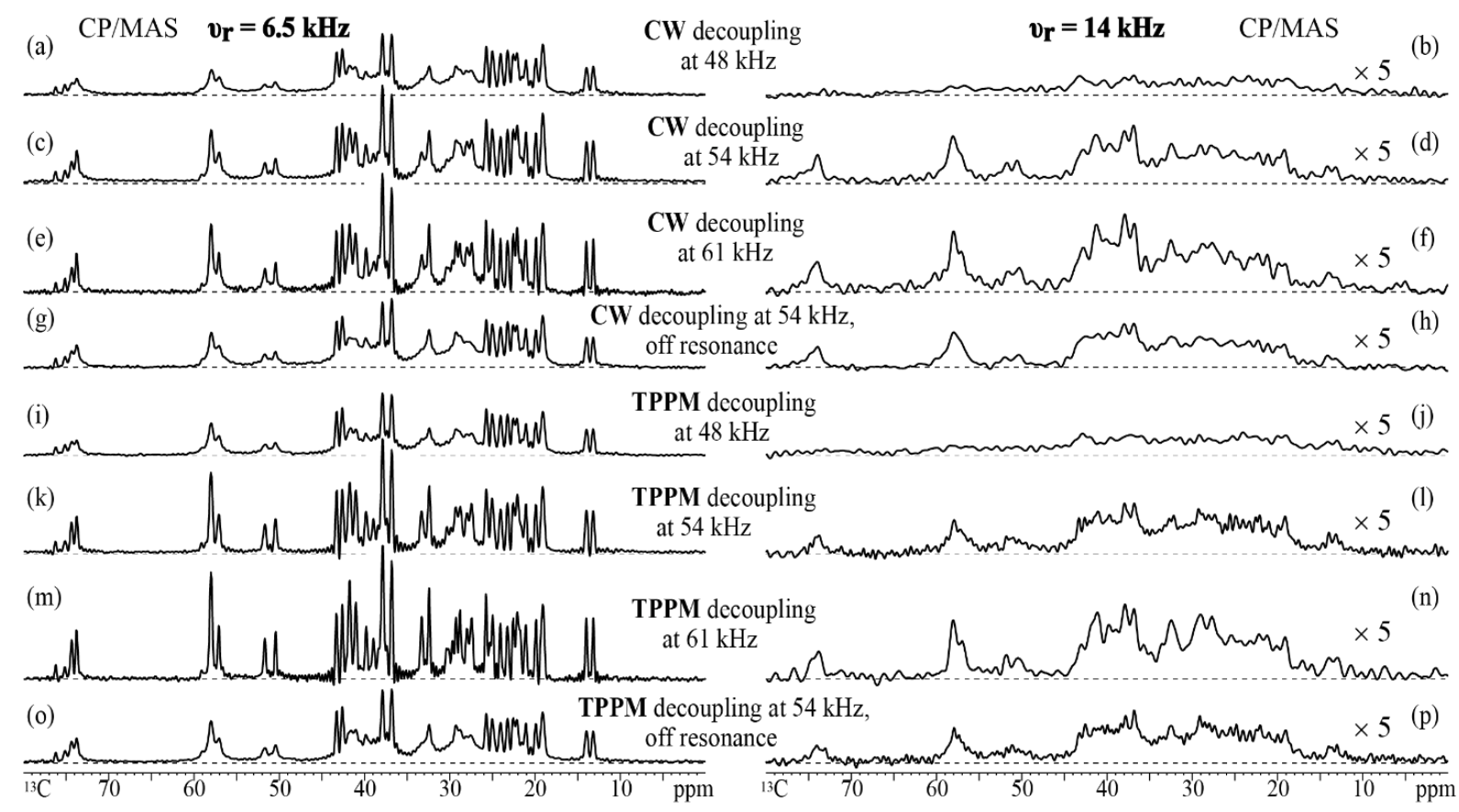

Figure 4. Mao et al. 


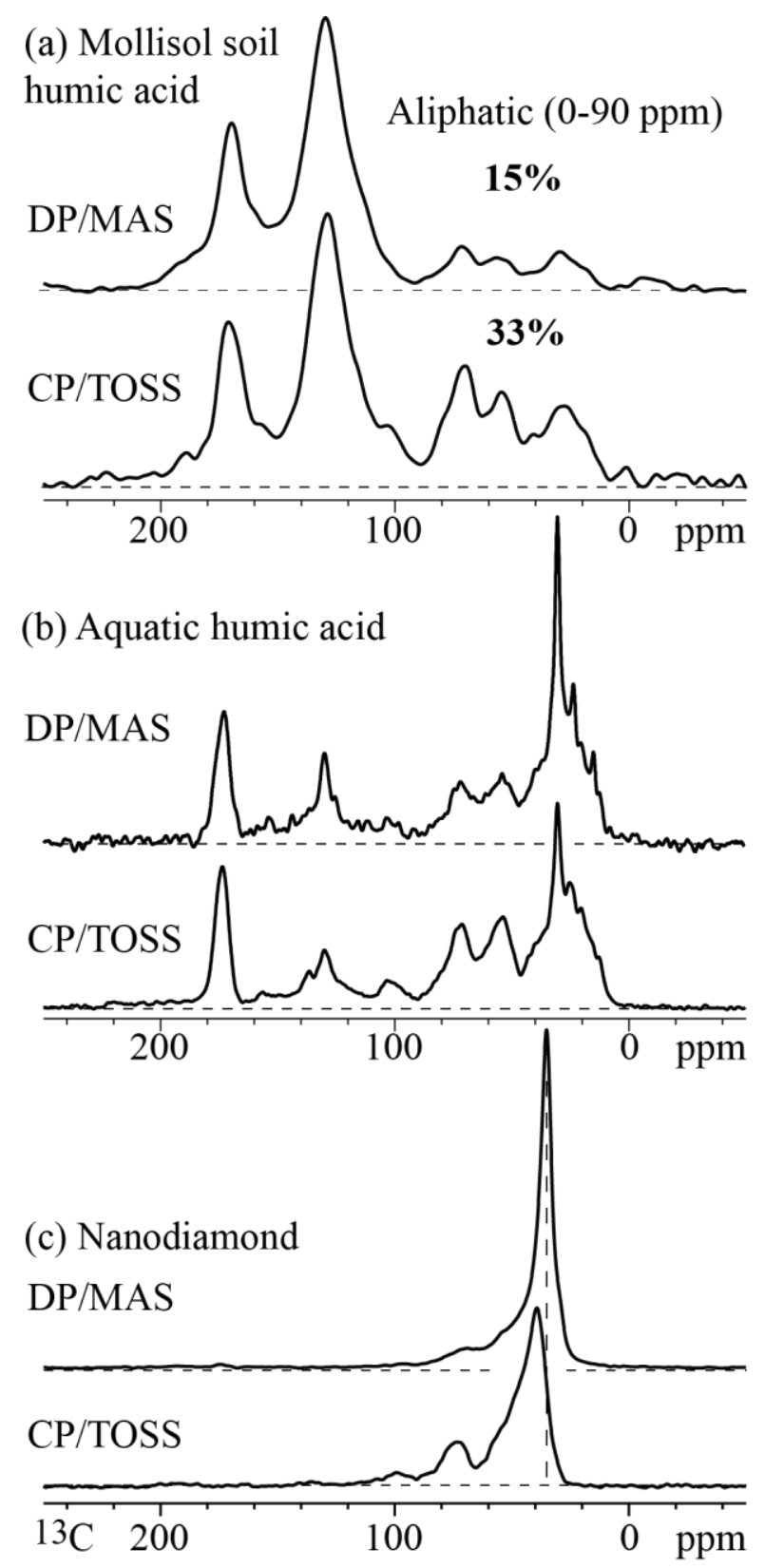

Figure 5. Mao et al. 

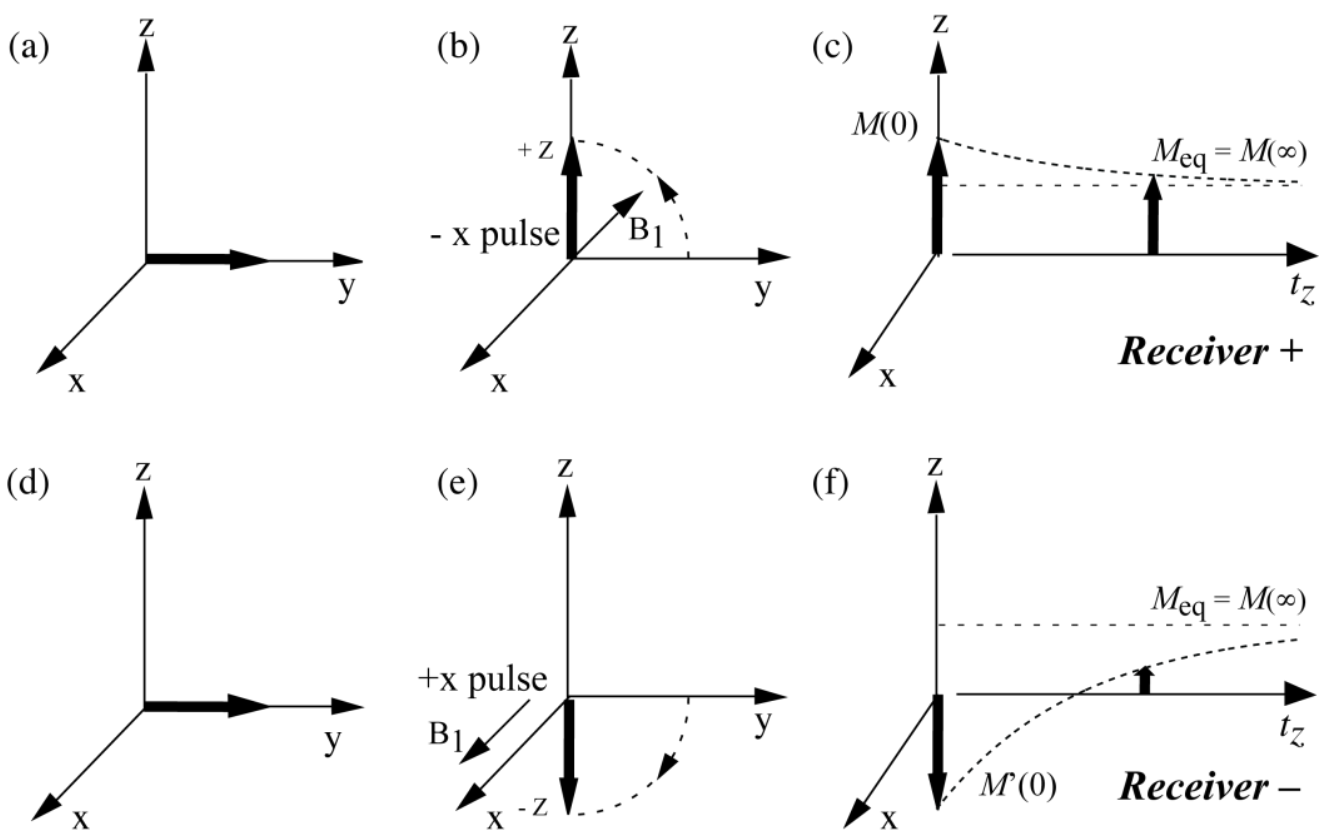

Fig.6. Mao et al. 


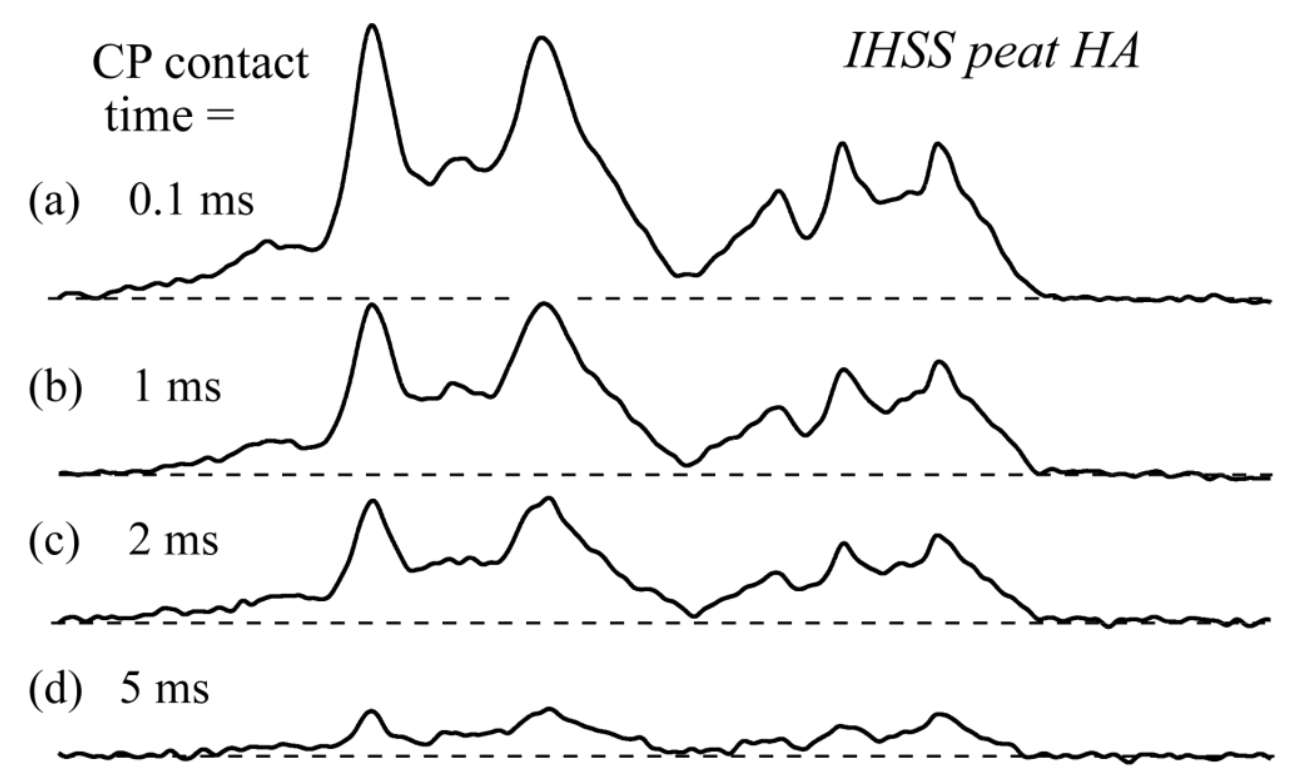

(e) $10 \mathrm{~ms}$

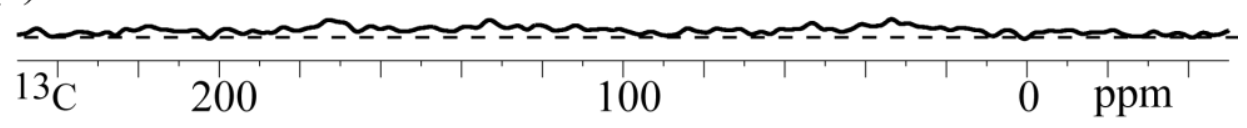

Figure 7 Mao et al. 

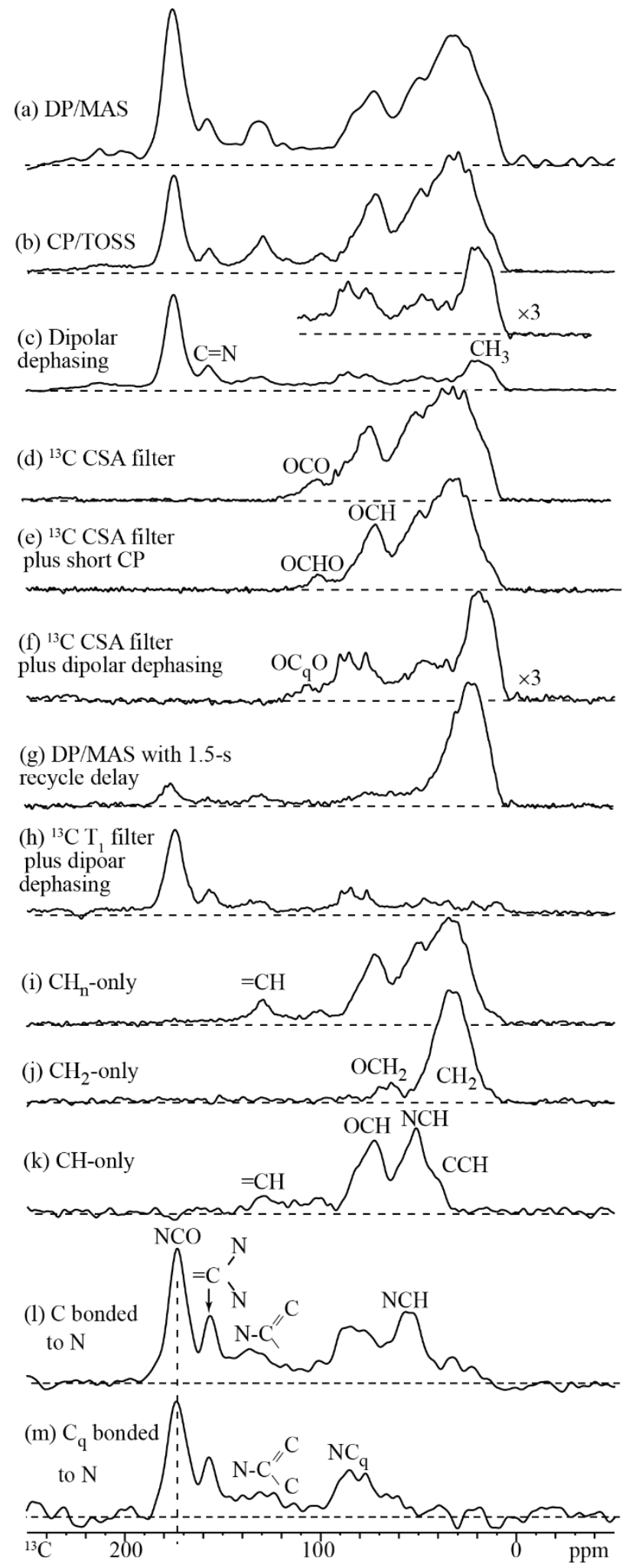

Figure 8. Mao et al. 


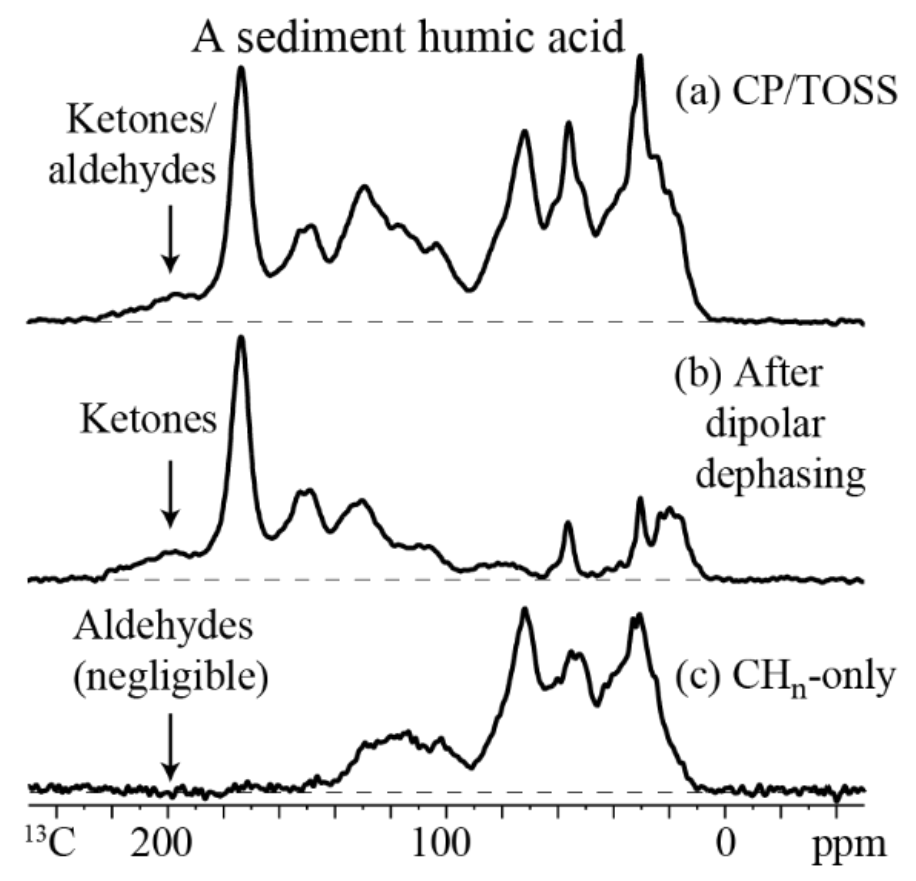

Figure 9. Mao et al.
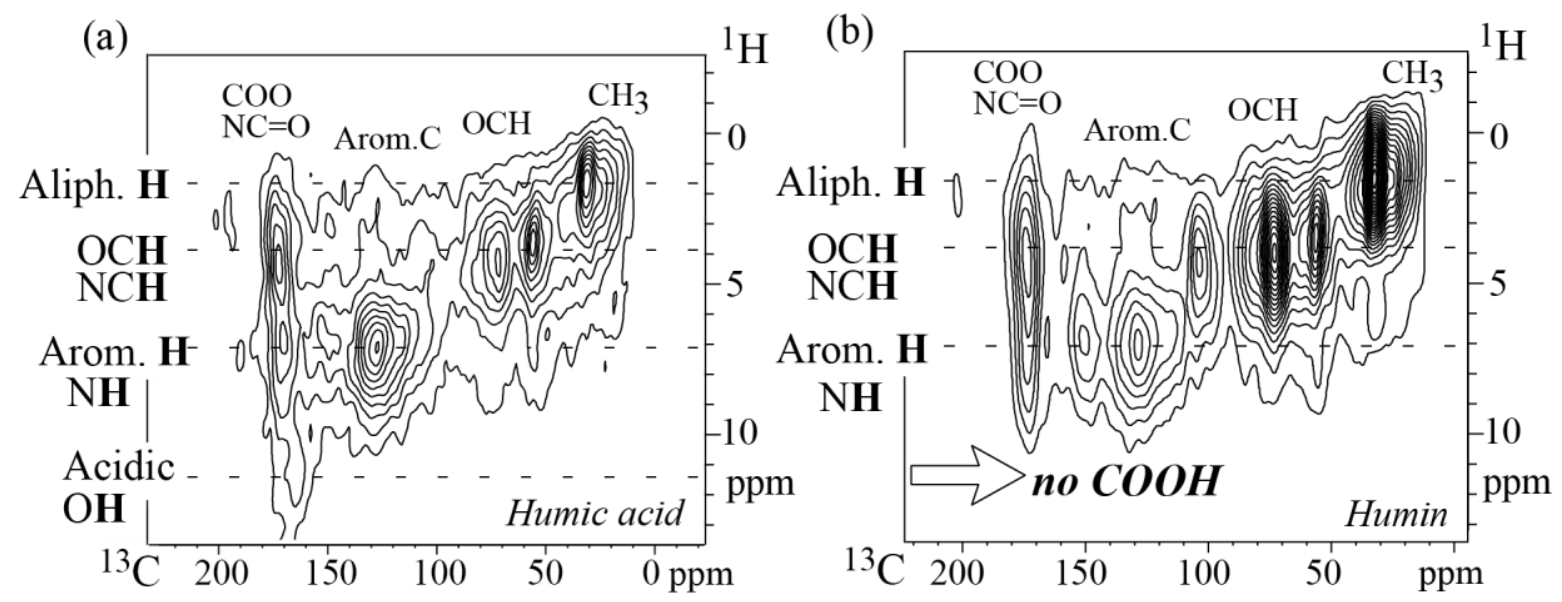

Figure 10 Mao et al. 


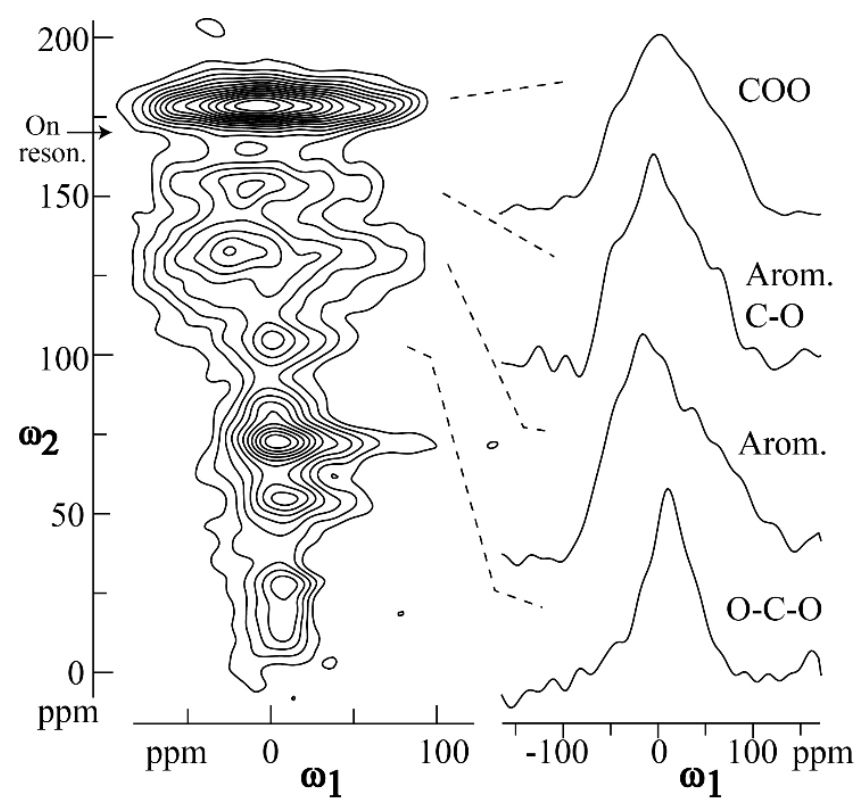

Figure 11. Mao et al. 

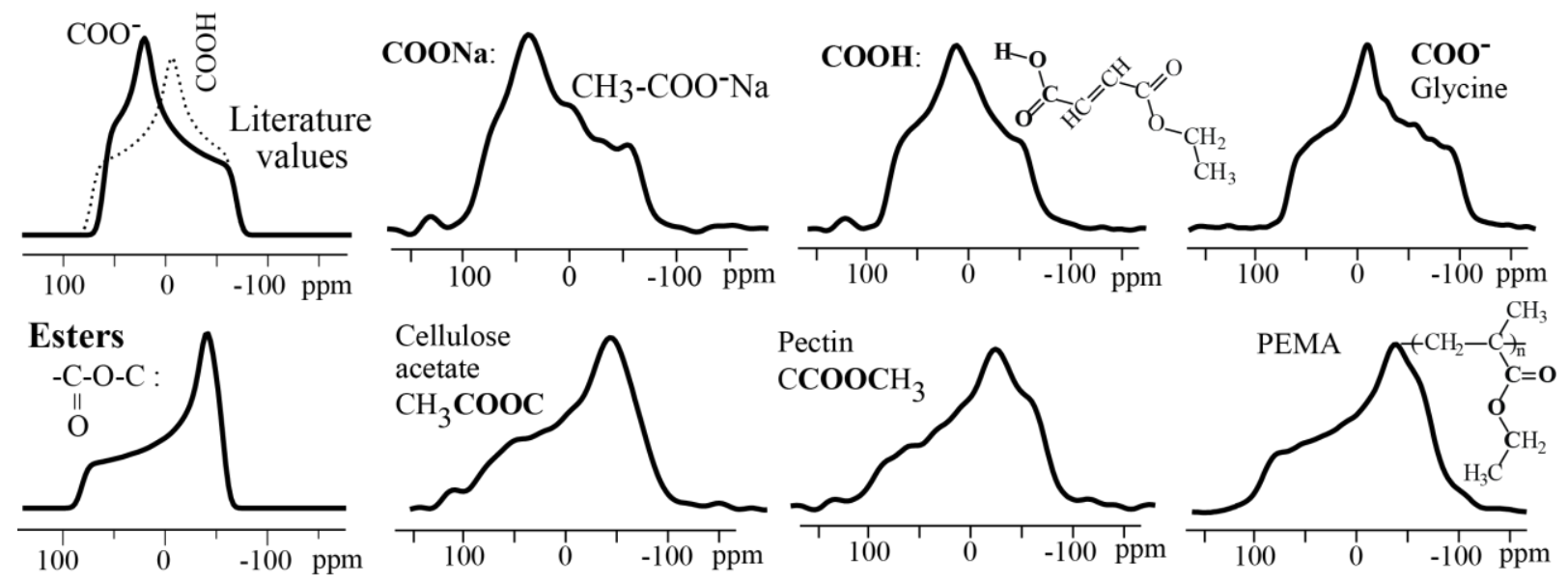

Figure 12. Mao et al.

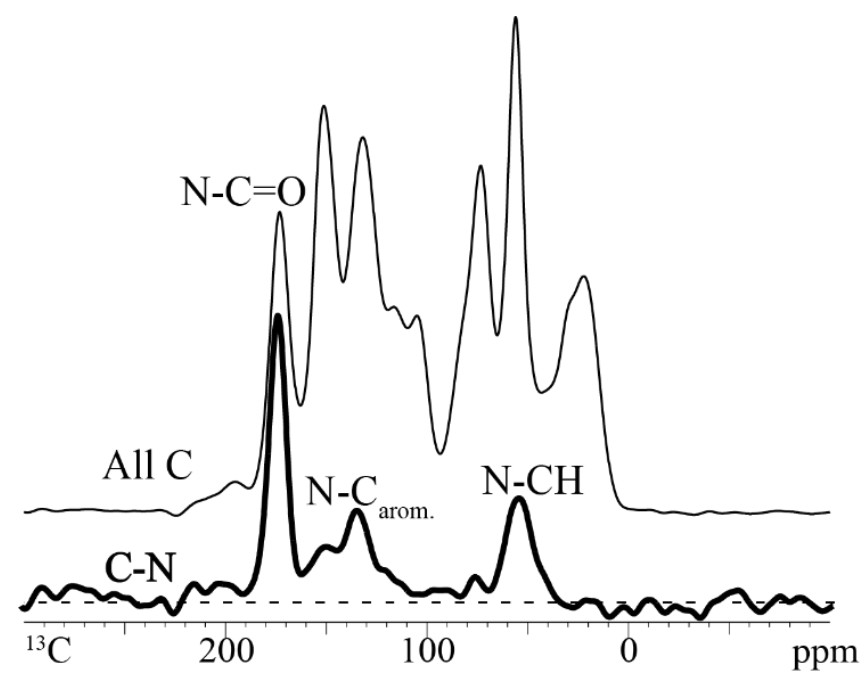

Figure 13. Mao et al. 


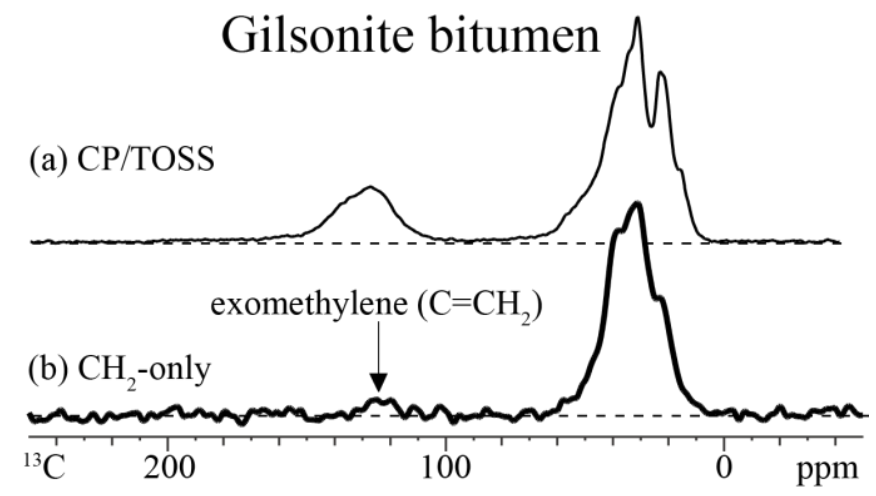

Figure 14. Mao et al.

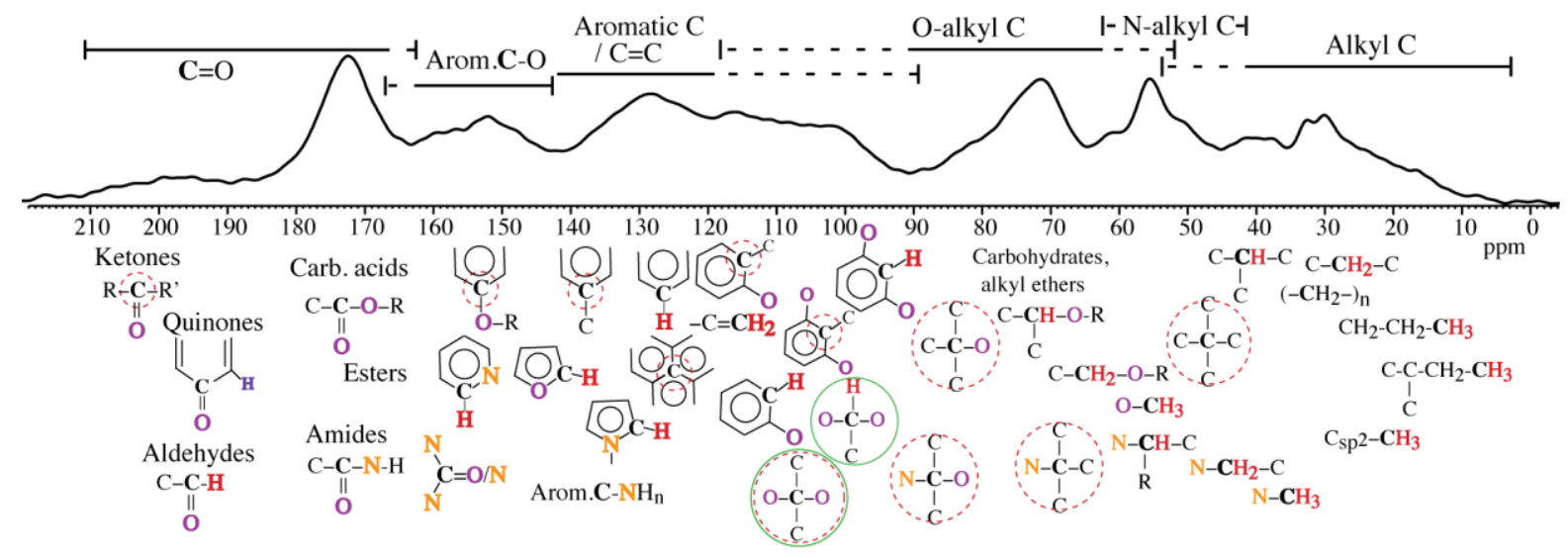

Figure 15. Mao et al. 


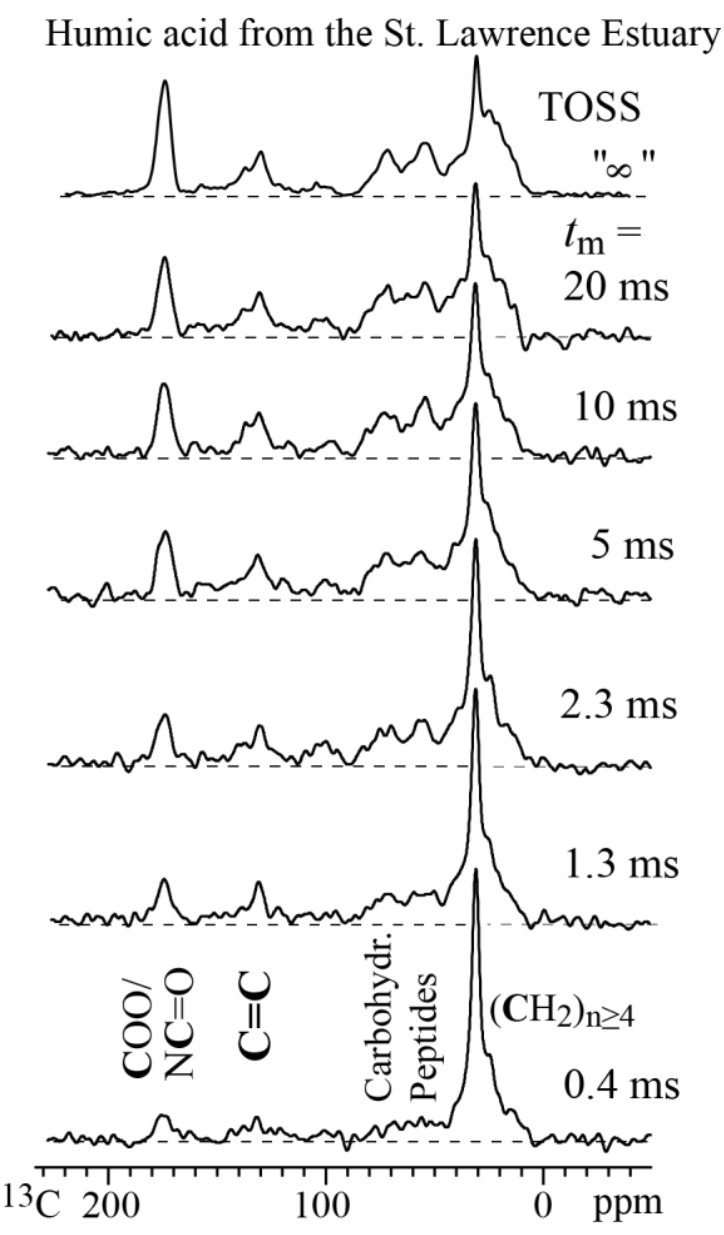

Figure 16. Mao et al. 

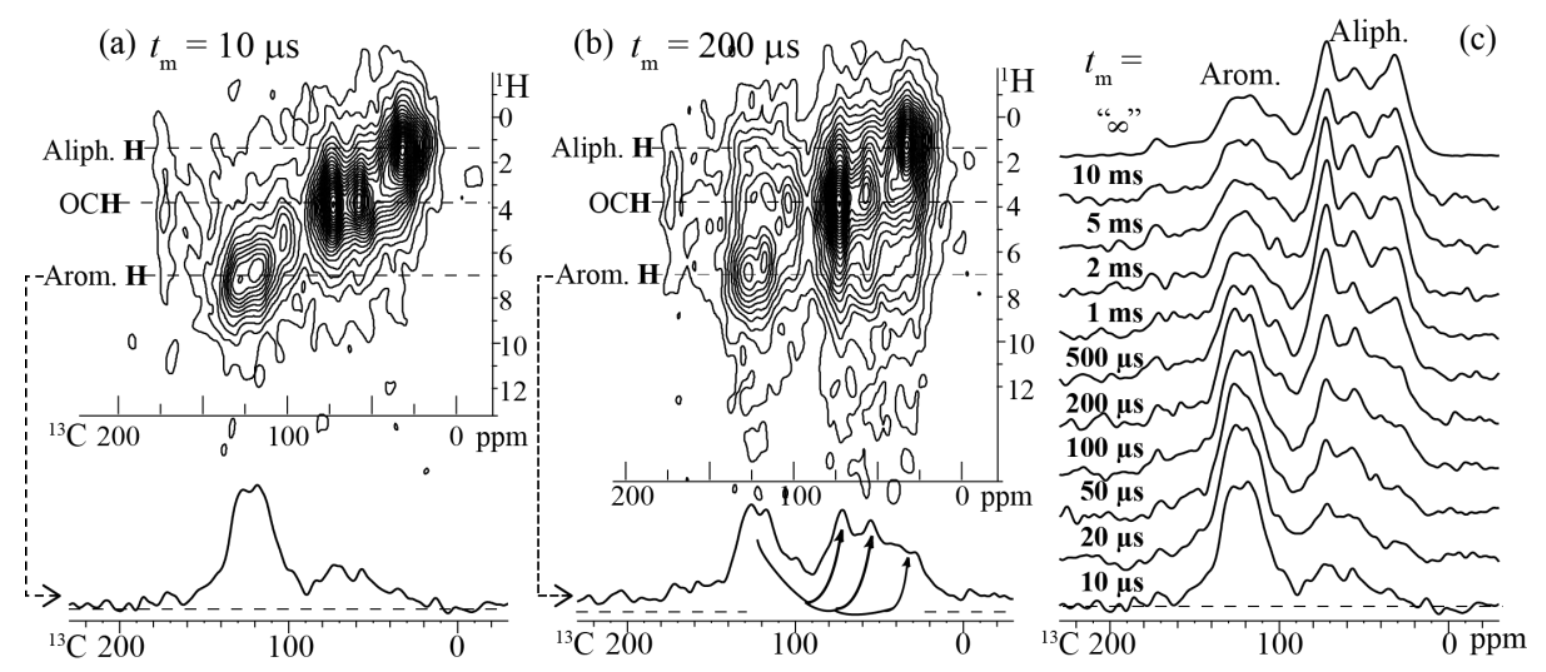

Figure 17. Mao et al. 

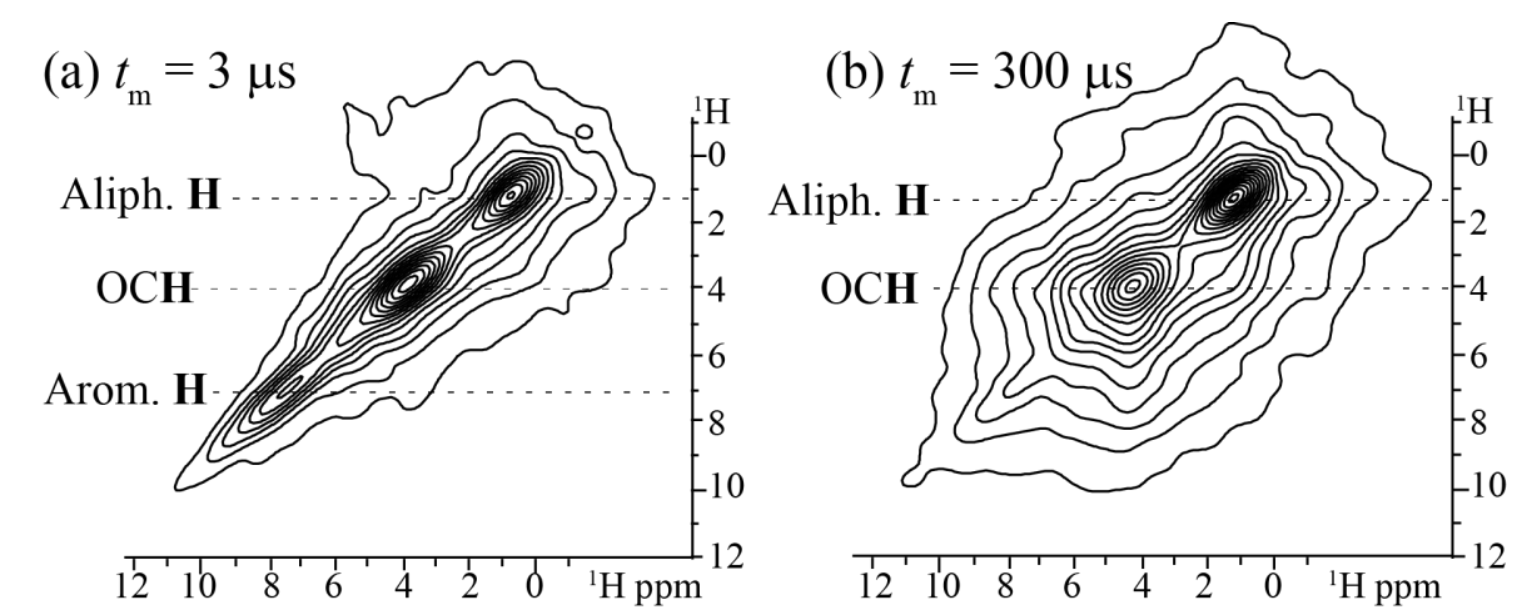

(c) $t_{\mathrm{m}}=1 \mathrm{~ms}$

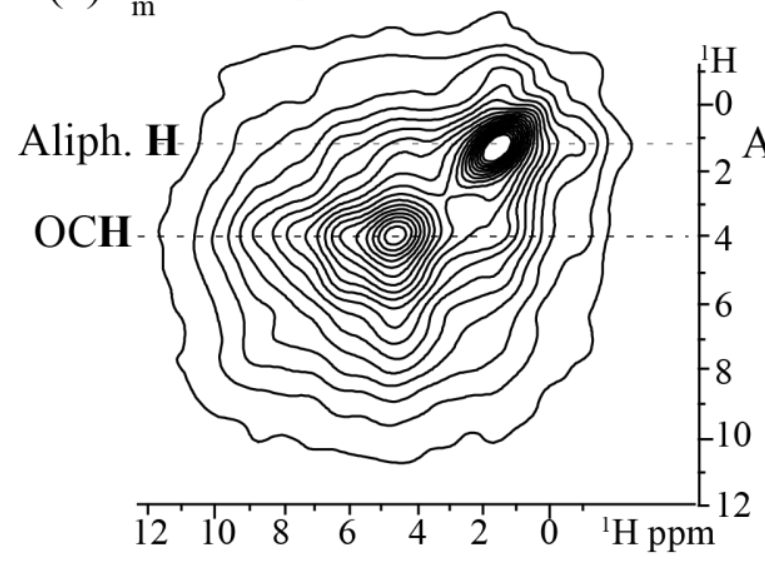

(d) $t_{\mathrm{m}}=10 \mathrm{~ms}$

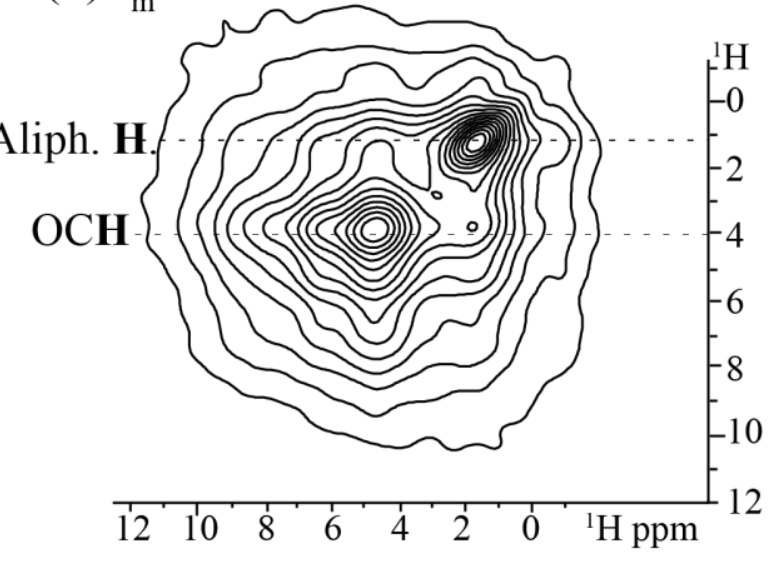

Figure 18. Mao et al. 


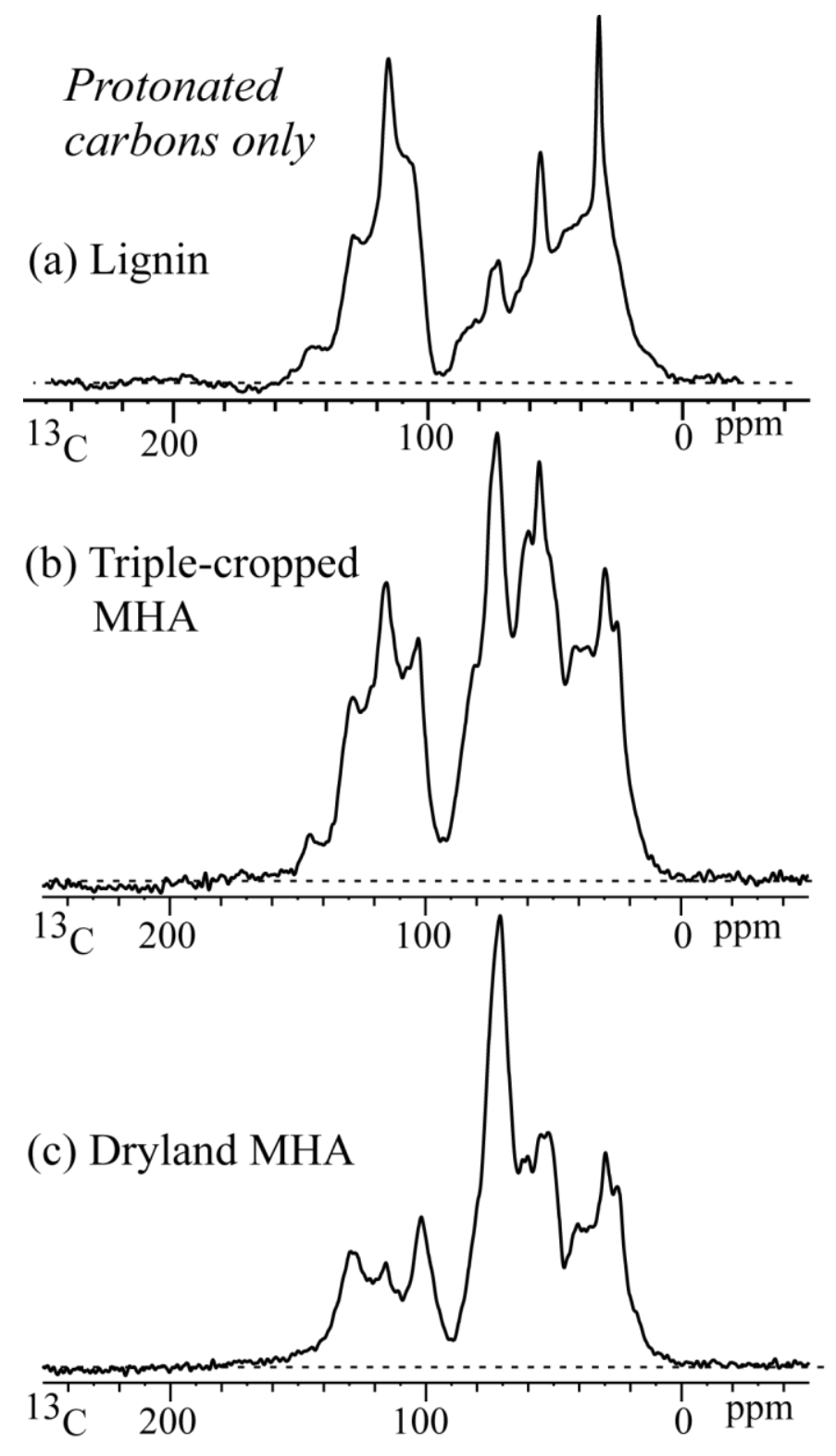

Figure 19. Mao et al. 


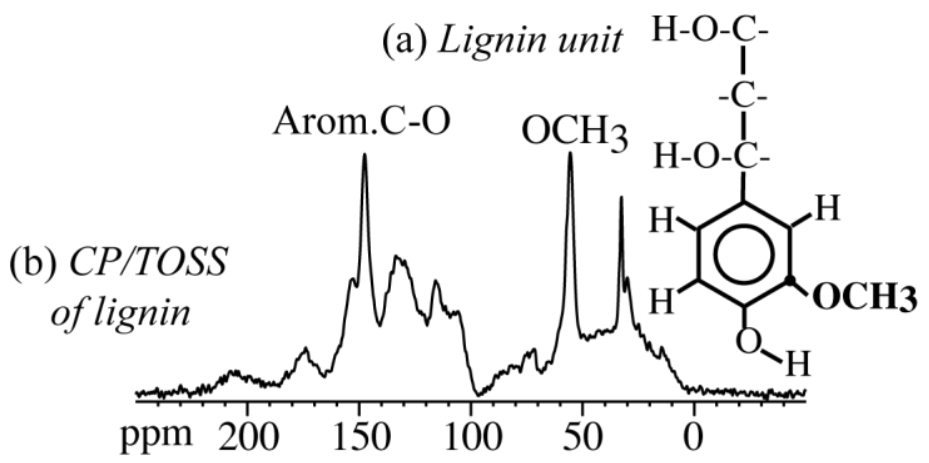

(c) 2D HETCOR of lignin

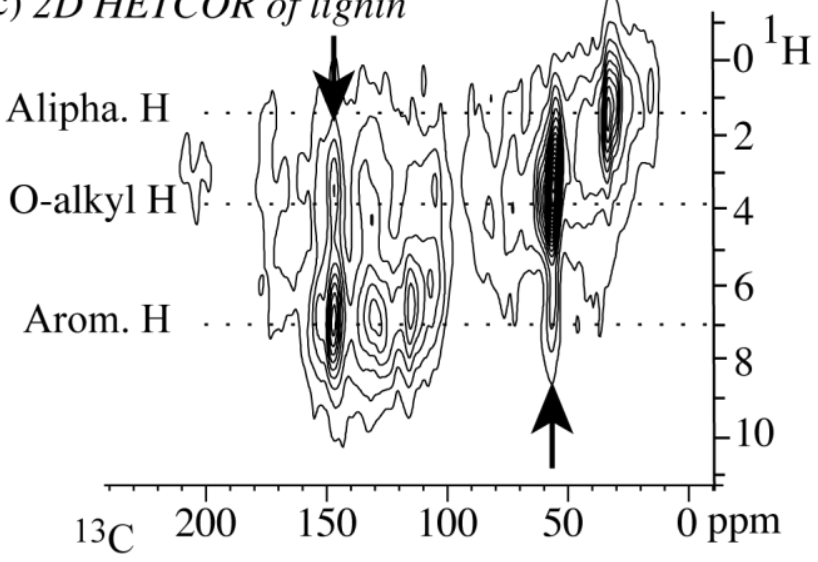

(d) 2D HETCOR of

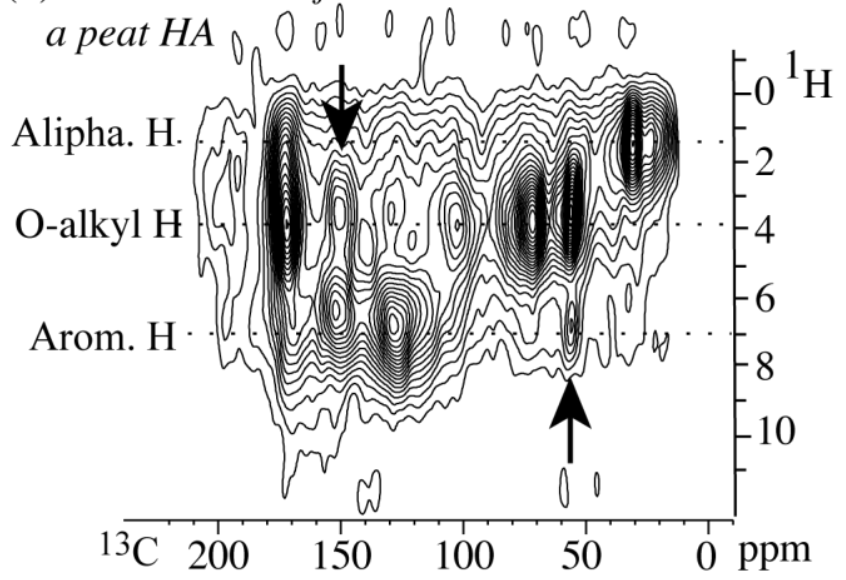

Figure 20 Mao et al. 

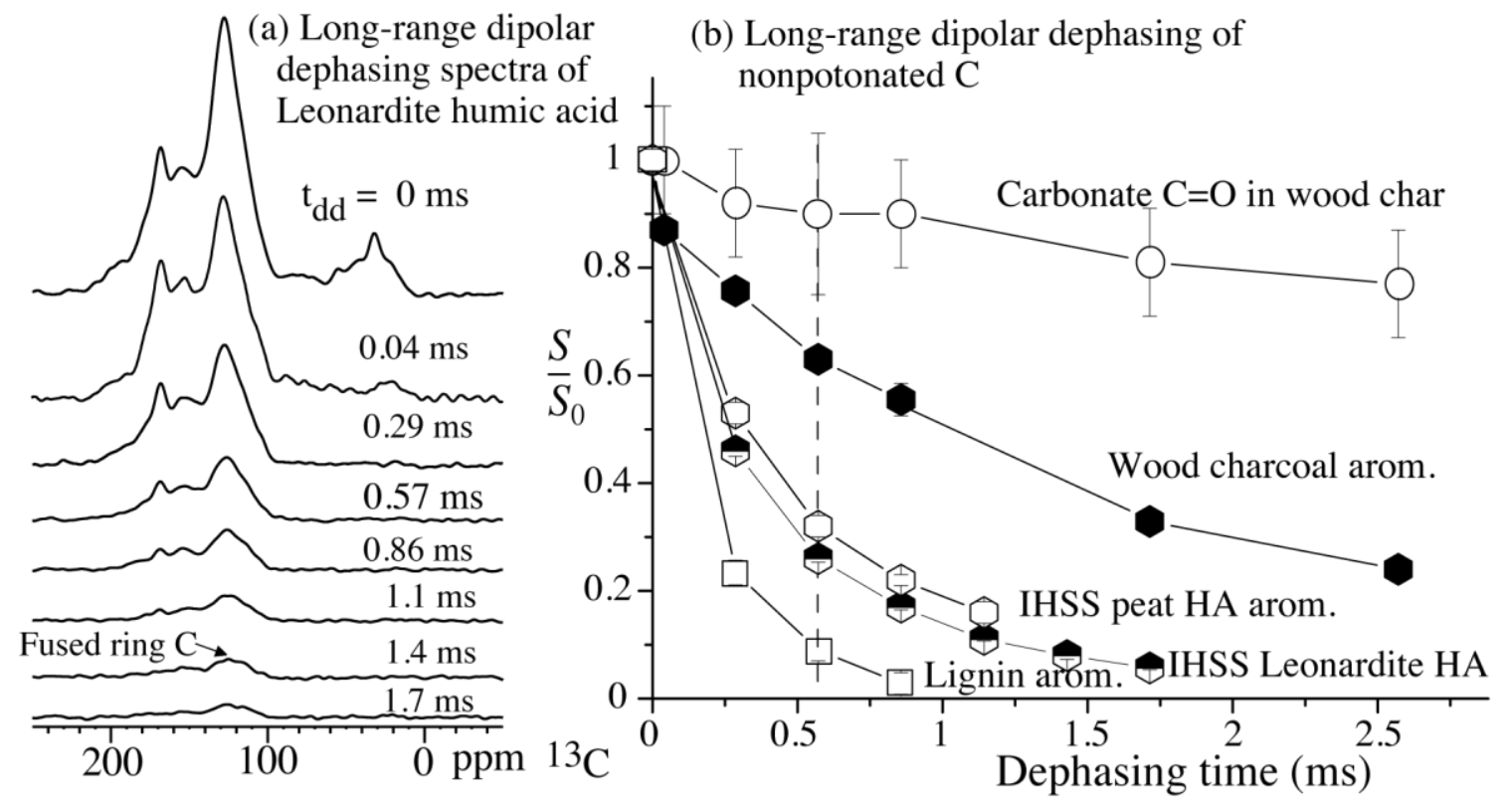

Figure 21. Mao et al. 


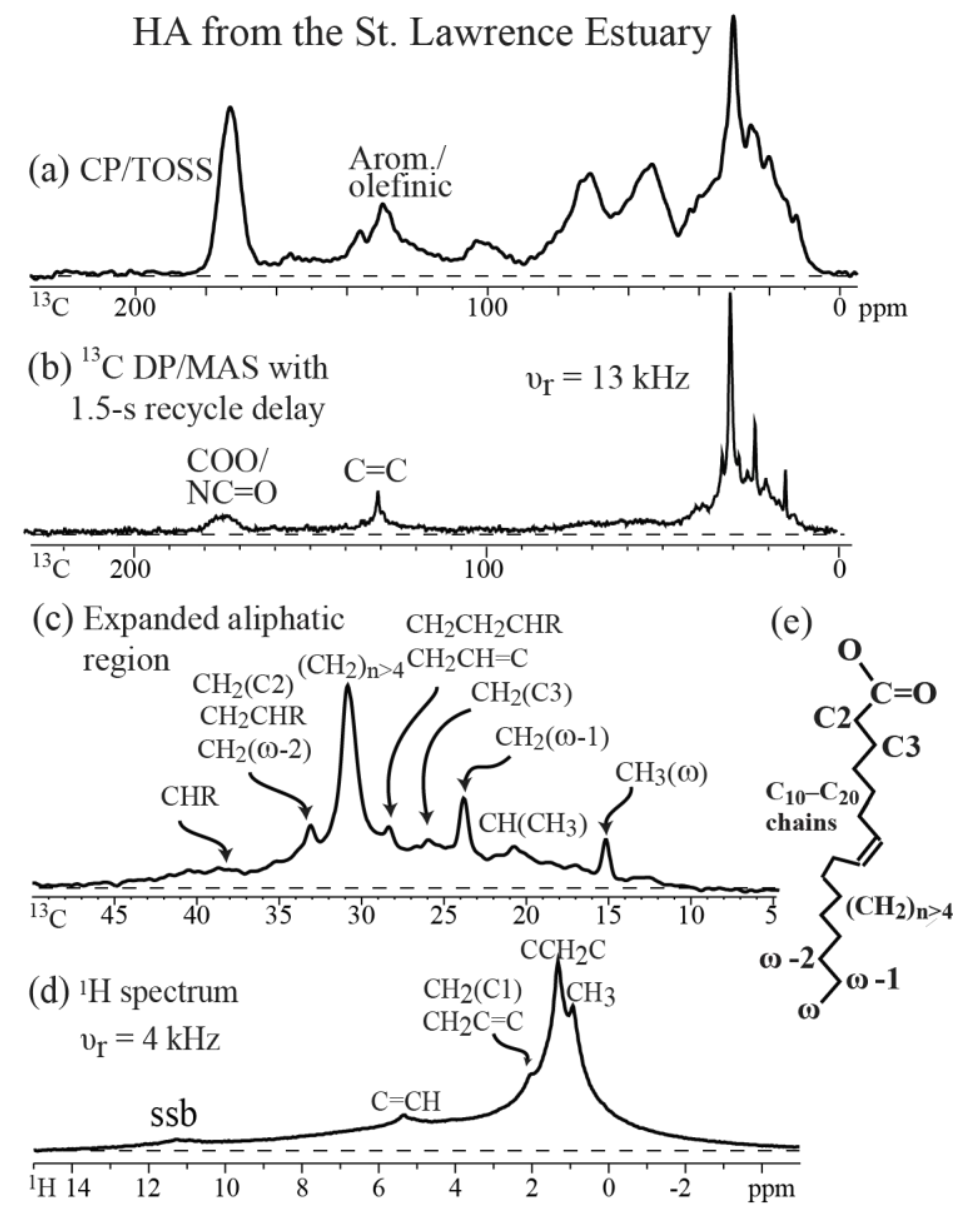

Figure 22. Mao et al. 

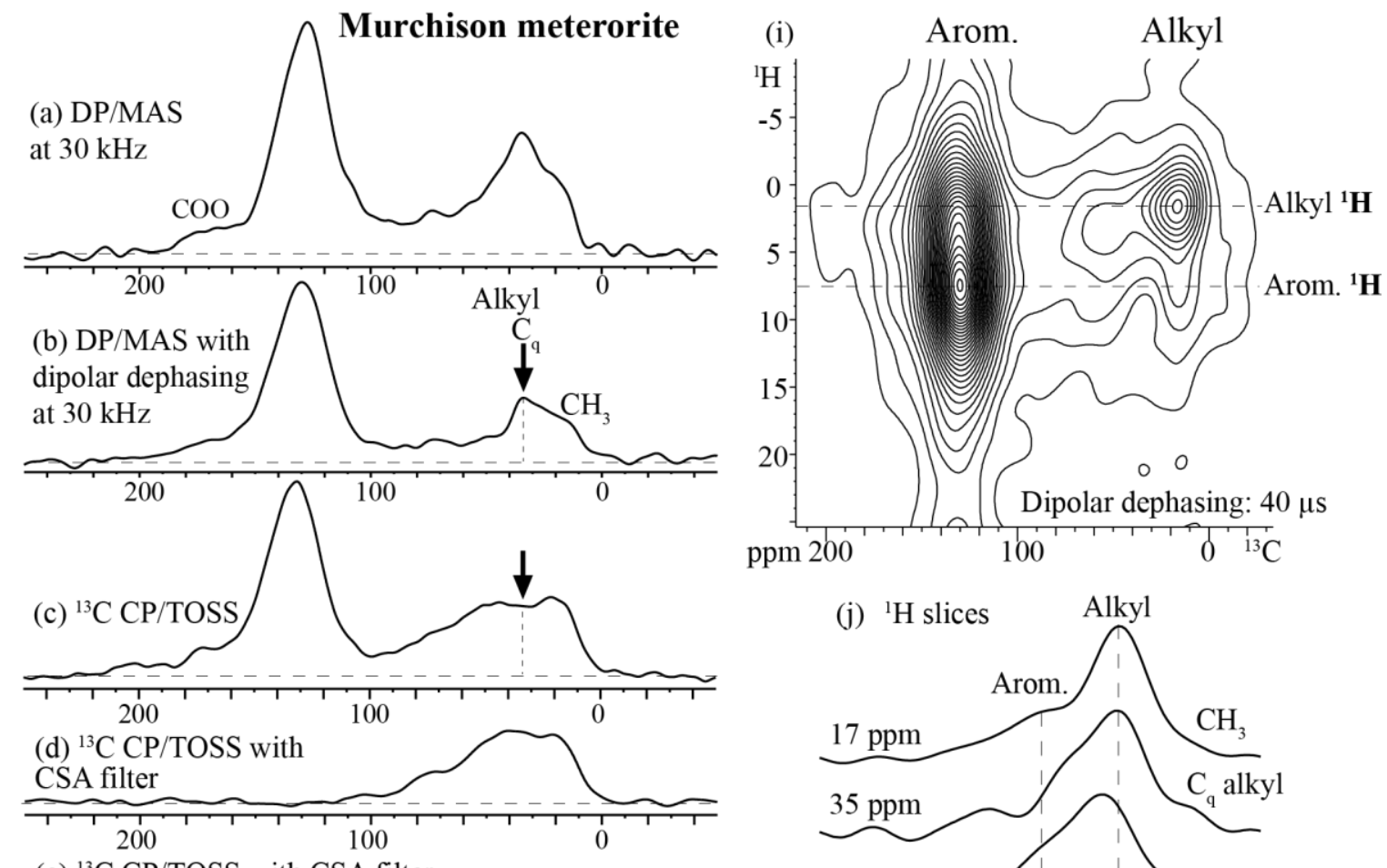

(e) ${ }^{13} \mathrm{C} \mathrm{CP} / \mathrm{TOSS}$ with CSA filter

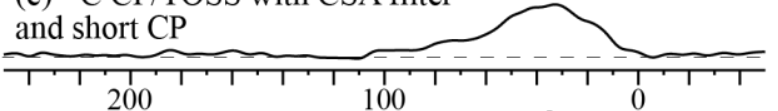
(f) ${ }^{13} \mathrm{C} \mathrm{CP} / \mathrm{TOSS}$ with CSA filter and dipolar dephasing
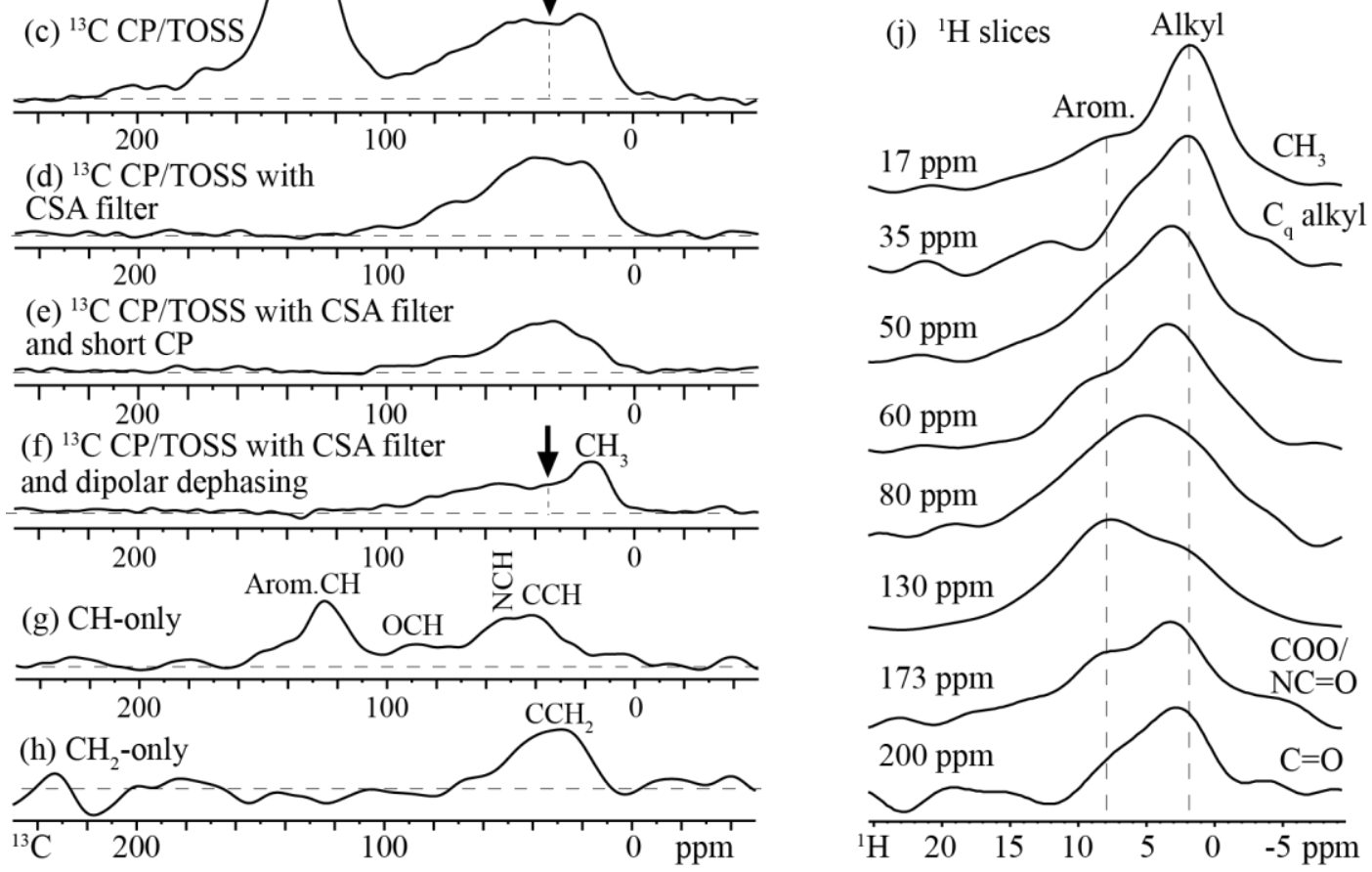

Figure 23. Mao et al. 


\section{Glossary of abbreviations}

APT: attached-proton test

CODEX: centerband-only detection of exchange

CP/MAS: cross polarization/magic angle spinning

CRAMPS: combined rotation and multiple-pulse spectroscopy

CSA: chemical shift anisotropy

CW: continuous-wave

DEPT: distortionless enhancement by polarization transfer

DOM: Dissolved organic matter

DP/MAS: direct polarization/magic angle spinning

FID: free induction decay

FSLG: frequency-switched Lee-Goldburg

HA: humic acid

HARDSHIP: heteronuclear recoupling with dephasing by strong homonuclear interactions of protons

HETCOR: heteronuclear correlation

HMBC: heteronuclear multiple bond coherence

HMQC: heteronuclear multiple quantum coherence

HSQC: heteronuclear single quantum coherence

HW8: Haeberlen-Waugh 8-pulse

IHSS: International Humic Substances Society

INEPT: insensitive nuclei enhanced by polarization transfer 
IR: infrared

MAT: magic angle turning

MHA: mobile humic acid

MQ: multiple-quantum

MREV: mansfield-Rhim-Elleman-Vaughn

multiple cross polarization: multiCP

NMR: nuclear magnetic resonance

NOM: natural organic matter

OC: organic carbon

POM: particulate organic matter

PSRE: proton spin relaxation editing approach

REAPDOR: rotational-echo adiabatic-passage double-resonance

REDOR: rotational-echo double-resonance

RIDER: relaxation-induced dipolar exchange with recoupling

S/N: signal-to-noise ratio

SOM: soil organic matter

SPIDER: saturation-pulse induced dipolar exchange with recoupling

TOSS: total suppression of sidebands

TPPM: two-pulse phase-modulated decoupling

TRAPDOR: transfer of populations in double resonance

WIMSE: windowless isotropic mixing for spectral editing

WIS: Wisconsin Range 


\section{References}

[1] F.J. Stevenson, Humus chemistry: genesis, composition, reactions John Wiley \& Sons, New York, 1994.

[2] M.H.B. Hayes, Emerging concepts of the compositions and structures of humic substances, in: M.H.B. Hayes, W.S. Wilson (Eds.) Humic Substances in Soils, Peats and Waters: Health and Environmental Aspects, The Royal Society of Chemistry, Cambridge, UK, 1997, pp. 3-30. [3] M.H.B. Hayes, Solvent systems for the isolation of organic components from soils, Soil Sci. Soc. Am. J., 70 (2006) 986-994.

[4] M.H.B. Hayes, C.E. Clapp, Humic substances: Considerations of compositions, aspects of structure, and environmental influences, Soil Sci., 166 (2001) 723-737.

[5] M.H.B. Hayes, P. MacCarthy, R.L. Malcolm, R.S. Swift, Humic substances II. In search of structure, John Wiley \& Sons, Chichester, 1989.

[6] J.A. Leenheer, Systematic approaches to comprehensive analyses of natural organic matter, Ann. Environ. Sci., 3 (2009) 1-130.

[7] J.J. Pignatello, Dynamic interactions of natural organic matter and organic compounds, J. Soils Sed., 12 (2012) 1241-1256.

[8] D. Laird, The charcoal vision: A win-win-win scenario for simultaneously producing bioenergy, permanently sequestering carbon, while improving soil and water quality, Agron. J., 100 (2008) 178-181.

[9] B.P. Tissot, D.H. Welte, Petroleum Formation and Occurrence, 2nd ed., Springer-Verlag, Berlin, 1984.

[10] S. Bilgen, Structure and environmental impact of global energy consumption, Renewable Sustainable Energy Rev., 38 (2014) 890-902.

[11] N. Baccile, C. Falcod, M.-M. Titirici, Characterization of biomass and its derived char using ${ }^{13} \mathrm{C}$-solid state nuclear magnetic resonance, Green Chem., 16 (2014).

[12] H.-R. Schulten, M. Schnitzer, Chemical model structures for soil organic matter and soils, Soil Sci., 162 (1997) 115-130.

[13] R. Sutton, G. Sposito, Molecular structure in soil humic substances: The new view, Environ. Sci. Technol., 39 (2005) 9009-9015.

[14] E.W. Tegelaar, J.W. de Leeuw, C. Saiz-Jimene, Possible origin of aliphatic moieties in humic substances, Sci. Total Environ., 81/82 (1989) 1-17.

[15] P.G. Hatcher, K.J. Dria, S. Kim, S.W. Frazier, Modern analytical studies of humic substances, Soil Sci., 166 (2001) 770-794.

[16] J.I. Hedges, G. Eglinton, P.G. Hatcher, D.L. Kirchman, C. Arnosti, S. Derenne, R.P. Evershed, I. Kögel-Knabner, J.W. de Leeuw, R. Littke, W. Michaelis, J. Rullkotter, The molecularly-uncharacterized component of nonliving organic matter in natural environments, Org. Geochem., 31 (2000) 945-958.

[17] N. Hertkorn, Environmental NMR: Solution - state methods, eMagRes, (2014) 55-74.

[18] A.J. Simpson, D.J. McNally, M.J. Simpson, NMR Spectroscopy in environmental research: From molecular interactions to global processes, Prog. Nucl. Magn. Reson. Spectrosc., 58 (2011) 97-175.

[19] K. Mopper, A. Stubbins, J.D. Ritchie, H.M. Bialk, P.G. Hatcher, Advanced instrumental approaches for characterization of marine dissolved organic matter: Extraction techniques, mass spectrometry, and nuclear magnetic resonance spectroscopy, Chem. Rev., 107 (2007) 419-442. 
[20] C.M. Preston, Environmental NMR-the early years, Magn. Reson. Chem., 53 (2015) 635647.

[21] R.L. Cook, Coupling NMR to NOM, Anal. Bioanal. Chem., 378 (2004) 1484-1503.

[22] P. Conte, R. Spaccini, A. Piccolo, State of the art of CPMAS C-13-NMR spectroscopy applied to natural organic matter, Prog. Nucl. Magn. Reson. Spectrosc., 44 (2004) 215-223. [23] R.E. Botto, R. Wilson, R.E. Winans, Evaluation of the reliability of solid ${ }^{13} \mathrm{C}$ NMR spectroscopy for the quantitative analysis of coals: study of whole coals and maceral concentrates, Energy Fuels, 1 (1987) 173-181.

[24] J. Mao, N. Chen, X. Cao, Characterization of humic substances by advanced solid state NMR spectroscopy: Demonstration of a systematic approach, Org. Geochem., 42 (2011) 891902.

[25] E.R. DeAzevedo, T.J. Bonagamba, D. Reichert, Molecular dynamics in solid polymers, Prog. Nucl. Magn. Reson. Spectrosc., 47 (2005) 137-164.

[26] K. Schmidt-Rohr, H.W. Spiess, Multidimensional solid-state NMR and polymers, Academic Press, London, 1994.

[27] R.L. Johnson, K. Schmidt-Rohr, Quantitative solid-state ${ }^{13}$ C NMR with signal enhancement by multiple cross polarization, J. Magn. Reson., 239 (2014) 44-49.

[28] C.M. Preston, Applications of NMR to soil organic matter analysis: History and prospects, Soil Sci., 161 (1996) 144-166.

[29] C.M. Preston, Carbon-13 solid-state NMR of soil organic matter - using the technique effectively, Can. J. Soil Sci., 81 (2001) 255-270.

[30] M.A. Wilson, NMR Techniques and Applications in Geochemistry and Soil Chemistry, Pergamon Press, London, 1987.

[31] M.A. Nanny, R.A. Minear, J.A. Leenheer, Nuclear Magnetic Resonance in Environmental Chemistry, Oxford University Press, New York, 1997.

[32] R.E. Botto, Y. Sanada, Magnetic Resonance of Carbonaceous Solids, American Chemical Society, Washington, DC, 1993.

[33] R.L. Wershaw, M.A. Mikita, NMR of Humic Substances and Coal, Lewis Publishers, Inc., Chelsea, MI, 1987.

[34] D.E. Axelson, Solid State Nuclear Magnetic Resonance of Fossil Fuels, Multiscience Publications Ltd., Canadian Government Publishing Center, Supply and Services Canada, 1985.

[35] I. Kögel-Knabner, ${ }^{13} \mathrm{C}$ and ${ }^{15} \mathrm{~N}$ NMR spectroscopy as a tool in soil organic matter studies, Geoderma, 80 (1997) 243-270.

[36] M. Duer, Introduction to Solid-State NMR Spectroscopy, Blackwell Publishing Inc., Malden, MA, 2004.

[37] A.C. Kolbert, D.P. Raleigh, R.G. Griffin, $\pi$ pulses and echo formation in magic-anglespinning NMR, J. Magn. Reson., 82 (1989) 483-491.

[38] J. Mao, W. Hu, G. Ding, K. Schmidt-Rohr, G. Davies, E.A. Ghabbour, B. Xing, Suitability of different ${ }^{13} \mathrm{C}$ solid-state NMR techniques in the characterization of humic acids, Int. J. Environ. Anal. Chem., 82 (2002) 183-196.

[39] G. Bodenhausen, R. Freeman, D.L. Turner, Suppression of artifacts in two-dimensional J spectroscopy, J. Magn. Reson. (1969), 27 (1977) 511-514.

[40] W.T. Dixon, Spinning-sideband-free and spinning-sideband-only NMR spectra in spinning samples, J. Chem. Phys., 77 (1982) 1800-1809. 
[41] M. Mehring, Principles of high resolution NMR in solids, Springer-Verlag, Berlin, New York, 1983.

[42] M. Ernst, Heteronuclear spin decoupling in solid-state NMR under magic-angle sample spinning, J. Magn. Reson., 162 (2003) 1-34.

[43] A.E. Bennett, C.M. Rienstra, M. Auger, K.V. Lakshmi, R.G. Griffin, Heteronuclear decoupling in rotating solids, J. Chem. Phys., 103 (1995) 6951-6958.

[44] M. Ernst, S. Bush, A.C. Kolbert, A. Pines, Second-order recoupling of chemical-shielding and dipolar-coupling tensors under spin decoupling in solid-state NMR, J. Chem. Phys., 105 (1996) 3387-3397.

[45] M. Carravetta, M. Eden, X. Zhao, A. Brinkmann, M.H. Levitt, Symmetry principles for the design of radiofrequency pulse sequences in the nuclear magnetic resonance of rotating solids Chemical Physical Letters, 321 (2000) 205-215.

[46] K. Takegoshi, J. Mizokami, T. T. Terao, H-1 decoupling with third averaging in solid NMR, Chemical Physical Letters, 341 (2001) 540-544.

[47] J.-D. Mao, K. Schmidt-Rohr, Accurate quantification of aromaticity and nonprotonated aromatic carbon fraction in natural organic matter by ${ }^{13} \mathrm{C}$ solid-state nuclear magnetic resonance, Environ. Sci. Technol., 38 (2004) 2680-2684.

[48] J.D. Mao, W.G. Hu, K. Schmidt-Rohr, G. Davies, E.A. Ghabbour, B.S. Xing, Quantitative characterization of humic substances by solid-state carbon-13 nuclear magnetic resonance, Soil Sci. Soc. Am. J., 64 (2000) 873-884.

[49] H. Knicker, Pyrogenic organic matter in soil: Its origin and occurrence, its chemistry and survival in soil environments, Quat. Int., 243 (2011) 251-263.

[50] J.I. Hedges, J.A. Baldock, Y. Gelinas, C. Lee, M. Peterson, S.G. Wakeham, Evidence for non-selective preservation of organic matter in sinking marine particles, Nature, 409 (2001) 801-804.

[51] R.J. Smernik, J.M. Oades, The use of spin counting for determining quantitation in solid state C-13 NMR spectra of natural organic matter 1. Model systems and the effects of paramagnetic impurities, Geoderma, 96 (2000) 101-129.

[52] E.M. Levin, S.L. Bud'ko, J.D. Mao, Y. Huang, K. Schmidt-Rohr, Effect of magnetic particles on NMR spectra of Murchison meteorite organic matter and a polymer-based model system, Solid State Nucl. Magn. Reson., 31 (2007) 63-71.

[53] J.A. Rice, Humin, Soil Science, 166 (2001) 848-857.

[54] G. Kervern, G. Pintacuda, Y. Zhang, E. Oldfield, C. Roukoss, E. Kuntz, E. Herdtweck, J.M. Basset, S. Cadars, A. Lesage, C. Coperet, L. Emsley, Solid-state NMR of a paramagnetic DIAD-Fe-II catalyst: Sensitivity, resolution enhancement, and structure-based assignments, J. Am. Chem. Soc., 128 (2006) 13545-13552.

[55] J.-D. Mao, L. Tremblay, J.P. Gagne, S. Kohl, J. Rice, K. Schmidt-Rohr, Humic acids from particulate organic matter in the Saguenay Fjord and the St. Lawrence Estuary investigated by advanced solid-state NMR, Geochim. Cosmochim. Acta, 71 (2007) 5483-5499

[56] X. Fang, J. Mao, E.M. Levin, K. Schmidt-Rohr, Nonaromatic core-shell structure of nanodiamond from solid-state NMR spectroscopy, J. Am. Chem. Soc., 131 (2009) 1426-1435. [57] D.A. Torchia, Measurement of proton-enhanced carbon-13 $\mathrm{T}_{1}$ values by a method which suppresses artifacts, J. Magn. Reson., 30 (1978) 613-616.

[58] G. Metz, X.L. Wu, S.O. Smith, Ramped-amplitude cross polarization in magic-anglespinning NMR J. Magn. Reson., Ser. A, 110 (1994) 219-227. 
[59] G. Metz, M. Ziliox, S.O. Smith, Towards quantitative CP-MAS NMR, Solid State Nucl. Magn. Reson., 7 (1996) 155-160.

[60] E.H. Novotny, M.H.B. Hayes, E.R. deAzevedo, T.J. Bonagamba, Characterisation of black carbon-rich samples by $\mathrm{C}-13$ solid-state nuclear magnetic resonance,

Naturwissenschaften, 93 (2006) 447-450.

[61] E.H. Novotny, E.R. deAzevedo, T.J. Bonagamba, T.J.F. Cunha, B.E. Madari, V.d.M. Benites, M.H.B. Hayes, Studies of the compositions of humic acids from Amazonian Dark Earth soils, Environmental Sciene \& Technology, 41 (2007) 400-405.

[62] R.L. Cook, C.H. Langford, Structural characterization of a fulvic acid and a humic acid using solid state ramp-CP-MAS C-13 nuclear magnetic resonance. , Environ. Sci. Technol., 32 (1998) 719-725.

[63] J.M. Anderson, R.L. Johnson, K. Schmidt-Rohr, B.H. Shanks, Solid state NMR study of chemical structure and hydrothermal deactivation of moderate-temperature carbon materials with acidic $\mathrm{SO}_{3} \mathrm{H}$ sites, Carbon, 74 (2014) 333-345.

[64] H. Yabuta, H. Naraoka, K. Sakanishi, H. Kawashima, Solid-state ${ }^{13}$ C NMR characterization of insoluble organic matter from Antarctic CM2 chondrites: Evaluation of the meteoritic alteration level, Meteorit. Planet. Sci., 40 (2005) 779-787.

[65] D.L. VanderHart, H. Retcofsky, Estimation of coal aromaticities by proton-decoupled carbon-13 magnetic resonance spectra of whole coals, Fuel, 55 (1976) 202-204.

[66] R.E. Botto, Fossil fuels in: D. M. Grant, R.K. Harris (Eds.) Encyclopedia of Nuclear Magnetic Resonance, John Wiley \& Sons, New York, 1996, pp. p 2101-2118.

[67] C.M. Preston, R.H. Newman, P. Rother, Using ${ }^{13}$ C CPMAS NMR to assess effects of cultivation on the organic matter of particle size fractions in a grassland soil, Soil Sci., 157 (1994) 26-35.

[68] C. Preston, R. Newman, A long-term effect of $\mathrm{N}$ fertilization on the ${ }^{13} \mathrm{C}$ CPMAS NMR of de-ashed soil humin in a second-growth Douglas-fir stand of coastal British Columbia, Geoderma, 68 (1995) 229-241.

[69] C. Keeler, G.E. Maciel, Quantitation in the solid-state ${ }^{13} \mathrm{C}$ NMR analysis of soil and organic soil fractions, Anal. Chem., 75 (2003) 2421-2432.

[70] X.W. Fang, T. Chua, K. Schmidt-Rohr, M.L. Thompson, Quantitative C-13 NMR of whole and fractionated Iowa Mollisols for assessment of organic matter composition, Geochim. Cosmochim. Acta, 74 (2010) 584-598.

[71] R.K. Harris, NMR studies of organic polymorphs \& solvates, Analyst, 131 (2006) 351373.

[72] K.W. Zilm, Spectral editing techniques: hydrocarbon solids, in: D.M. Grant, R.K. Harris (Eds.) Encyclopedia of Nuclear Magnetic Resonance, New York: John Wiley and Sons., 1996, pp. $4498-4504$.

[73] S.J. Opella, M.H. Frey, Selection of nonprotonated carbon resonances in solid-state nuclear magnetic resonance, J. Am. Chem. Soc., 101 (1979) 5854-5856.

[74] P.A. Tishmack, D.E. Bugay, S.R. Byrn, Solid-state nuclear magnetic resonance spectroscopy - pharmaceutical applications J. Pharm. Sci., 92 (2003) 441-474.

[75] R.R. Ernst, G. Bodenhausen, A. Wokaun, Principles of Nuclear Magnetic Resonance in One and Two Dimensions, Clarendon Press: Oxford, 1987.

[76] T. Terao, H. Mirua, A. Saika, Simplification and assignment of carbon-13 spectra by using J-resolved NMR spectroscopy in solids, J. Am. Chem. Soc., 104 (1982) 528-5229. 
[77] A. Lesage, S. S., L. Emsley, Carbon-13 spectral editing in solid-state NMR using heteronuclear scalar coupling, J. Am. Chem. So.c, 120 (1998) 7095-7100.

[78] D. Sakellariou, A. Lesage, L. Emsley, Spectral editing in solid-state NMR using scalar multiple quantum filters, J. Magn. Reson., 151 (2001) 40-47.

[79] D.P. Burum, A. Bielecki, WIMSE, a new spectral editing technique for CPMAS solidstate NMR, J. Magn. Reson., 95 (1991) 184-190.

[80] X. Wu, S.T. Burns, K.W. Zilm, Spectral editing in CPMAS NMR. Generating subspectra based on proton multiplicities, J. Magn. Reson., Ser. A, 111 (1994) 29-36.

[81] X. Wu, K.W. Zilm, Methylene-only subspectrum in CPMAS NMR, J. Magn. Reson., Ser. A, 104 (1993) 119-122.

[82] X.L. Wu, K.W. Zilm, Complete spectral editing in CPMAS NMR, J. Magn. Reson., Ser A, 102 (1993) 205-213.

[83] S.T. Burns, X.L. Wu, K.W. Zilm, Improvement of spectral editing in solids: A sequence for obtaining $(\mathrm{CH})-\mathrm{C}-13+(\mathrm{CH} 2)-\mathrm{C}-13$-only C-13 spectra, J. Magn. Reson., 143 (2000) 352359.

[84] R. Sangill, N. Rastrup-Andersen, H. Bildsoe, H.J. Jakobsen, N.C. Nielsen, Optimized spectral editing of ${ }^{13} \mathrm{C}$ MAS NMR spectra of rigid solids using cross-polarization methods, J. Magn. Reson., Series A, 107 (1994) 67-78.

[85] J.D. Mao, K. Schmidt-Rohr, Methylene spectral editing in solid-state ${ }^{13} \mathrm{C}$ NMR by threespin coherence selection, J. Magn. Reson., 176 (2005) 1-6.

[86] J.Z. Hu, J.K. Harper, C. Taylor, R.J. Pugmire, D.M. Grant, Modified spectral editing methods for C-13 CP/MAS experiments in solids, J. Magn. Reson., 142 (2000) 326-330.

[87] C. Keeler, G.E. Maciel, ${ }^{13}$ C NMR spectral editing of humic material, J. Mol. Struct., 550 (2000) 297-305.

[88] S.H. Mao, X.A. Mao, Z.H. Xu, J.Z. Hu, B.L. Yang, L.Y. Li, C.H. Ye, P. Saffigna, CP/MAS C-13 spectral editing of dried pine leaves, Solid State Nucl. Magn. Reson., 12 (1998) 31-36.

[89] C. Forte, A. Piazzi, S. Pizzanelli, G. Certini, CP MAS C-13 spectral editing and relative quantitation of a soil sample, Solid State Nucl. Magn. Reson., 30 (2006) 81-88.

[90] K. Saalwächter, R. Graf, H.W. Spiess, Recoupled polarization-transfer methods for solidstate ${ }^{1} \mathrm{H}^{13}{ }^{13} \mathrm{C}$ heteronuclear correlation in the limit of fast MAS, J. Magn. Reson., 148 (2001) 398-418.

[91] E. E. De Vita, L. L. Frydman, Spectral editing in C-13 MAS NMR under moderately fast spinning conditions J. Magn. Reson., 148 (2001) 327-337.

[92] I. Fischbach, T. Pakula, P. Minkin, A. Fechtenkotter, K. Mullen, H.W. Spiess, K.

Saalwachter, Structure and dynamics in columnar discotic materials: A combined X-ray and solid-state NMR study of hexabenzocoronene derivatives, J. Phys. Chem. B, 106 (2002) 64086418.

[93] K. Mao, G.J. Kennedy, S.M. Althaus, M. Pruski, Spectral editing in ${ }^{13}$ C solid-state NMR at high magnetic field using fast MAS and spin-echo dephasing, Solid State Nucl. Magn.

Reson., 47 (2012) 19-22.

[94] J. Peng, L. Frydman, Spectral editing in solid-state MAS NMR using chemical-shiftanisotropy-dephasing techniques, J. Magn. Reson., Ser A, 113 (1995) 247-250.

[95] N. Zumbulyadis, Selective carbon excitation and the detection of spatial heterogeneity in cross- polarization magic-angle-spinning NMR, J. Magn. Reson., 53 (1983) 486-494. 
[96] R.S. Aujla, R.K. Harris, K.J. Packer, M. Parameswaran, B.J. Say, A. Bunn, E.A. Cudby, Discriminatory experiments in high-resolution ${ }^{13} \mathrm{C}$ NMR of solid polymers, Polym. Bull., 8 (1982) 253-259.

[97] M.J. Sullivan, G.E. Maciel, Structural resolution in the C-13 nuclear magnetic resonance spectrometric analysis of coal by cross polarization magic angle spinning, Anal. Chem., 54 (1982) 1606-1615.

[98] P. Tekely, M.R. Vignon, Proton T1 and T2 relaxation times of wood components using C13 CP MAS NMR, J. Polym. Sci., Part C: Polym. Lett., 25 (1987) 257-261.

[99] R.H. Atalla, D.L. Vanderhart, Native Cellulose - A composite of 2 distinct crystalline forms, Science, 223 (1984) 283-285.

[100] R.H. Newman, J.A. Hemmingson, Determination of the degree of cellulose crystallinity in wood by C-13 nuclear-magnetic-resonance spectroscopy, Holzforschung, 44 (1990) 351355.

[101] H.R. Tang, Y.L. Wang, P.S. Belton, C-13 CPMAS studies of plant cell wall materials and model systems using proton relaxation-induced spectral editing techniques, Solid State Nucl. Magn. Reson., 15 (2000) 239-248.

[102] R.H. Newman, L.M. Condron, Separating subspectra from cross-polarization magicangle spinning nuclear magnetic resonance spectra by proton spin relaxation editing, Solid State Nucl. Magn. Reson., 4 (1995) 259-266.

[103] C.M. Preston, R.J. Smernik, R.F. Powers, J.G. McColl, T.M. McBeath, The decomposition of windrowed, chipped logging slash and tree seedling response: A plant growth and nuclear magnetic resonance spectroscopy study, Org. Geochem., 42 (2011) 936946.

[104] M. Schilling, W.T. Cooper, Effects of chemical treatments on the quality and quantitative reliability of solid-state C-13 NMR spectroscopy of mineral soils, Anal. Chim. Acta, 508 (2004) 207-216.

[105] A. Golchin, P. Clarke, J.M. Oades, The heterogeneous nature of microbial products as shown by solid-state C-13 CP/MAS NMR spectroscopy, Biogeochemistry 34 (1996) 71-97. [106] M. Pichler, H. Knicker, I. Kögel-Knabner, Changes in the chemical structure of municipal solid waste during composting as studied by solid-state dipolar dephasing and PSRE C-13 NMR and solid-state N-15 NMR spectroscopy, Environ. Sci. Technol., 34 (2002) 40344038 .

[107] R.J. Smernik, J.M. Oades, Effects of added paramagnetic ions on the C-13 CP MAS NMR spectrum of a de-ashed soil Gerdoma, 89 (1999) 219-248.

[108] J. Keeler, Understanding NMR Spectroscopy, John Wiley \& Sons, Ltd, United Kingdom, 2010.

[109] J.-D. Mao, L.S. Hundal, M.L. Thompson, K. Schmidt-Rohr, Correlation of poly(methylene)-rich aliphatic domains in humic substances with sorption of a nonpolar organic contaminant, phenanthrene, Environ. Sci. Technol., 36 (2002) 929-936.

[110] A. Rawal, X.Q. Kong, Y. Meng, J.U. Otaigbe, K. Schmidt-Rohr, Reduced crystallinity and mobility of Nylon-6 confined near the organic-inorganic interface in a phosphate glass-rich nanocomposite detected by H-1-C-13 NMR, Macromolecules, 44 (2011) 8100-8105. [111] R.C. Zhang, Y.Z. Chen, T.H. Chen, P.C. Sun, B.H. Li, D.T. Ding, Accessing structure and dynamics of mobile phase in organic solids by real-time T-1C filter PISEMA NMR spectroscopy, J. Phys. Chem. A, 116 (2012) 979-984. 
[112] J.-D. Mao, K. Schmidt-Rohr, Separation of aromatic-carbon ${ }^{13}$ C NMR signals from dioxygenated alkyl bands by a chemical-shift-anisotropy filter, Solid State Nucl. Magn. Reson., 26 (2004) 36-45.

[113] K. Schmidt-Rohr, J.D. Mao, Efficient CH-group selection and identification in ${ }^{13} \mathrm{C}$ solidstate NMR by dipolar DEPT and ${ }^{1} \mathrm{H}$ chemical-shift filtering, J. Am. Chem. Soc., 124 (2002) 13938-13948.

[114] J.D. Mao, K. Schmidt-Rohr, Recoupled long-range C-H dipolar dephasing in solid-state NMR, and its use for spectral selection of fused aromatic rings, J. Magn. Reson., 162 (2003) 217-227.

[115] K. Schmidt-Rohr, J.D. Mao, Selective observation of nitrogen-bonded carbons in solidstate NMR by saturation-pulse induced dipolar exchange with recoupling, Chem. Phys. Lett., 359 (2002) 403-411.

[116] X. Fang, J. Mao, R.M. Cory, D.M. McKnight, K. Schmidt-Rohr, ${ }^{15} \mathrm{~N}$ and ${ }^{13} \mathrm{C}\left\{{ }^{14} \mathrm{~N}\right\} \mathrm{NMR}$ investigation of the major nitrogen - containing segment in an aquatic fulvic acid: Evidence for a hydantoin derivative, Magn. Reson. Chem., 49 (2011) 775-780.

[117] T. Duncan, Chemical Shift Tensors, Farragut, Madison, WI, (1997).

[118] E.R. deAzevedo, W.-G. Hu, T.J. Bonagamba, K. Schmidt-Rohr, Principles of

centerband-only detection of exchange in solid-state NMR, and extentions to four-time CODEX, J. Chem. Phys., 112 (2000) 8988-9001.

[119] D. Doddrell, D. Pegg, M.R. Bendall, Distortionless enhancement of NMR signals by polarization transfer, J. Magn. Reson. (1969), 48 (1982) 323-327.

[120] W.K. Rhim, D. Elleman, R. Vaughan, Analysis of multiple pulse NMR in solids, The Journal of Chemical Physics, 59 (1973) 3740-3749.

[121] R.J. Smernik, J.M. Oades, Solid-state ${ }^{13} \mathrm{C}$ dipolar dephasing experiments for quantifying protonated and non-protonated carbon in soil organic matter and model systems, Eur. J. Soil

Sci., 52 (2001) 103-120.

[122] T. Gullion, J. Schaefer, Rotational-echo double-resonance NMR, J. Magn. Reson. (1969), 81 (1989) 196-200.

[123] R. Newman, Chemical-shift anisotropies can dominate dephasing in "dipolar dephasing" CP/MAS NMR experiments, J. Magn. Reson. (1969), 96 (1992) 370-375.

[124] L.B. Alemany, D.M. Grant, T.D. Alger, R.J. Pugmire, Cross polarization and magic angle sample spinning NMR spectra of model organic compounds. 3. Effect of the carbon-13proton dipolar interaction on cross polarization and carbon-proton dephasing, J. Am. Chem. Soc., 105 (1983) 6697-6704.

[125] K. Schmidt-Rohr, A. Rawal, X.W. Fang, A new NMR method for determining the particle thickness in nanocomposites, using T-2,T-H-selective $\mathrm{X}\{\mathrm{H}-1\}$ recoupling, J. Chem. Phys., 126 (2007) Article Number: 054701

[126] M.H. Levitt, R. Freeman, NMR population inversion using a composite pulse, J. Magn. Reson. (1969), 33 (1979) 473-476.

[127] N. Sinha, K. Schmidt-Rohr, M. Hong, Compensation for pulse imperfections in rotational-echo double-resonance NMR by composite pulses and EXORCYCLE, J. Magn. Reson., 168 (2004) 358-365.

[128] X. Mao, Z. Xu, R. Luo, N.J. Mathers, Y. Zhang, P.G. Saffigna, Nitrate in soil humic acids revealed by ${ }^{14} \mathrm{~N}$ nuclear magnetic resonance spectroscopy, Soil Res., 40 (2002) 717-726. 
[129] N. Mathers, X. Mao, P. Saffigna, Z. Xu, S. Berners-Price, M. Perera, Recent advances in the application of ${ }^{13} \mathrm{C}$ and ${ }^{15} \mathrm{~N}$ NMR spectroscopy to soil organic matter studies, Soil Res., 38 (2000) 769-787.

[130] H. Knicker, P.G. Hatcher, A.W. Scaroni, Solid-State ${ }^{15}$ N NMR spectroscopy of coal, Energy Fuels, 9 (1995) 999-1002.

[131] H. Knicker, R. Fründ, H.-D. Lüdemann, The chemical nature of nitrogen in native soil organic matter, Naturwissenschaften, 80 (1993) 219-221.

[132] K.A. Thorn, L.G. Cox, N-15 NMR spectra of naturally abundant nitrogen in soil and aquatic natural organic matter samples of the International Humic Substances Society, Org. Geochem., 40 (2009) 484-499.

[133] C.P. Grey, W.S. Veeman, A.J. Vega, Rotational echo ${ }^{14} \mathrm{~N} /{ }^{13} \mathrm{C} /{ }^{1} \mathrm{H}$ triple resonance solid state nuclear magnetic resonance: A probe of ${ }^{13} \mathrm{C}-{ }^{14} \mathrm{~N}$ internuclear distances, J. Chem. Phys., 98 (1993) 7711-7724.

[134] T. Gullion, Measurement of dipolar interactions between spin-12 and quadrupolar nuclei by rotational-echo, adiabatic-passage, double-resonance NMR, Chem. Phys. Lett., 246 (1995) 325-330.

[135] K. Saalwächter, K. Schmidt-Rohr, Relaxation-induced dipolar exchange with recoupling - An MAS NMR method for determining heteronuclear distances without irradiating the second spin, J. Magn. Reson., 145 (2000) 161-172.

[136] T. Gullion, J. Schaefer, Detection of weak heteronuclear dipolar coupling by rotationalecho double-resonance nuclear magnetic resonance, Adv. Magn. Reson., 13 (1989) 57-83. [137] S.F. Liu, J.D. Mao, K. Schmidt-Rohr, A robust technique for two-dimensional separation of undistorted chemical-shift anisotropy powder patterns in magic-angle-spinning NMR, J. Magn. Reson., 155 (2002) 15-28.

[138] H.-R. Schulten, M. Schnitzer, The chemistry of soil organic nitrogen: a review, Biol. Fertil. Soils 26 (1998) 1-5.

[139] D.C. Olk, Organic forms of soil nitrogen. Agronomy Monograph 49., in: J.S.S.a.W.R. Raun (Ed.) Nitrogen in Agricultural Systems, ASA, CSSA, SSSA, Madison, WI, 2008, pp. 57100.

[140] C.M. Preston, R. J.A., S.P. Mathur, M. Lévesque, Application of solution and solid-state multinuclear NMR to a peat-based composting systems for fish and crab scrap., Can. J. Spectrosc., 31 (1986) 63-69.

[141] K.A. Thorn, K. Kennedy, R. . N-15 NMR investination of the covalent binding of reduced TNT amines to soil humic acid, model compounds, and lignocellulose Environ. Sci. Technol., 17 (2002) 3787-3796

[142] I. Kögel-Knabner, Analytical approaches for characterizing soil organic matter, Org. Geochem., 31 (2000) 609-625.

[143] G. Almendros, R. Fründ, F.J. González-Vila, K. Haider, H. Knicker, H.-D. Lüdemann, Analysis of carbon-13 and nitrogen-15 CPMAS NMR-spectra of soil organic matter and composts, FEBS Lett., 282 (1991) 119-121.

[144] R.J. Smernik, J.A. Baldock, Does solid-state ${ }^{15}$ N NMR spectroscopy detect all soil organic nitrogen? , Biogeochemistry, 75 (2005) 507-528.

[145] R.J. Smernik, J.A. Baldock, Solid-state ${ }^{15} \mathrm{~N}$ NMR analysis of highly ${ }^{15} \mathrm{~N}$-enriched plant materials, Plant Soil, 275 (2005) 271-283. 
[146] S.R. Kelemen, M. Afeworki, M.L. Gorbaty, P.J. Kwiatek, M.S. Solum, J.Z. Hu, R.J. Pugmire, XPS and N-15 NMR study of nitrogen forms in carbonaceous solids, Energy Fuels, 16 (2002) 1507-1515.

[147] N. Mahieu, D. Olk, E. Randall, Accumulation of heterocyclic nitrogen in humified organic matter: a ${ }^{15} \mathrm{~N}$ - NMR study of lowland rice soils, Eur. J. Soil Sci., 51 (2000) 379-389. [148] C.M. Preston, B.S. Rauthan, C.A. Rodger, J.A. Ripmeester, A hydrogen-1, carbon-13 and nitrogen-15 nuclear magnetic resonance study of p-benzoquinone polymers incorporating amino nitrogen compounds ("synthetic humic acids"), Soil Sci., 134 (1982) 277-293.

[149] L.M. Benzing-Purdie, M.V. Cheshire, B.L. Williams, G.P. Sparling, C.I. Ratcliffe, J.A. Ripmeester, Fate of ${ }^{15} \mathrm{~N}$ glycine in peat as determined by ${ }^{13} \mathrm{C}$ and ${ }^{15} \mathrm{~N}$ CP-MAS NMR spectroscopy., J. Agric. Food. Chem, 34 (1986) 170-176.

[150] M.V. Cheshire, B.L. Williams, L.M. Benzing-Purdie, C.I. Ratcliffe, J.A. JRipmeester, Use of NMR spectroscopy to study transformation of nitrogenous substances during incubation of peat, Soil Use Manage., 6 (1990) 90-92.

[151] S.N. Zhuo, Q.X. Wen, Nitrogen forms in synthetic humic acids using nitrogen-15 nuclear magnetic resonance technique, Pedosphere 3(1993) 193-200.

[152] P.W. Clinton, R.H. Newman, R.B. Allen, Immobilization of ${ }^{15} \mathrm{~N}$ in forest litter studied by ${ }^{15}$ N CPMAS NMR spectroscopy, Eur. J. Soil Sci., 46 (1995) 551-556.

[153] R.J. DiCosty, D.P. Weliky, S.J. Anderson, E.A. Paul, ${ }^{15}$ N-CPMAS nuclear magnetic resonance spectroscopy and biological stability of soil organic nitrogen in whole soil and particle-size fractions, Org. Geochem., 34 (2003) 1635-1650.

[154] A. Potthast, R. R. Schiene, K. Fisher, Structural investigations of N-modified lignins by

${ }^{15} \mathrm{~N}-\mathrm{NMR}$ spectroscopy and possible pathways for formation of nitrogen containing compounds related to lignin. , Holzforschung, 50 (1996) 554-562.

[155] C.N. Bedrock, M.V. Cheshire, B.L. Williams, I. Solntseva, S.J. Chapman, J.A. Chudek, B.A. Goodman, Identification of nitrogenous components of fungal and bacterial origin immobilized in decomposing wheat straw by NMR spectroscopy using ${ }^{15} \mathrm{~N}$ CPMAS, Soil Biol. Biochem., 30 (1998) 113-115.

[156] K. Schmidt-Rohr, J.D. Mao, D.C. Olk, Nitrogen-bonded aromatics in soil organic matter and their implications for a yield decline in intensive rice cropping, Proc. Nat. Acad. Sci. U.S.A., 101 (2004) 6351-6354.

[157] J.-D. Mao, R.M. Cory, D.M. McKnight, K. Schmidt-Rohr, Characterization of a nitrogen-rich fulvic acid and its precursor algae by solid-state NMR, Org. Geochem., 38 (2007) 1277-1292.

[158] X. Fang, K. Schmidt-Rohr, Fate of the amino acid in glucose-glycine melanoidins investigated by solid-state nuclear magnetic resonance (NMR), J. Agric. Food. Chem., 57 (2009) 10701-10711.

[159] J.R. Helms, X. Kong, E. Salmon, P.G. Hatcher, K. Schmidt-Rohr, J. Mao, Structural characterization of gilsonite bitumen by advanced nuclear magnetic resonance spectroscopy and ultrahigh resolution mass spectrometry revealing pyrrolic and aromatic rings substituted with aliphatic chains, Org. Geochem., 44 (2012) 21-36.

[160] A. Bielecki , D.P. Burum, D.M. Rice , F.E. Karasz, Solid-state two-dimensional carbon13-proton correlation (HETCOR) NMR spectrum of amorphous poly(2,6-dimethyl-pphenylene oxide) (PPO), Macromolecules, 24 (1991) 4820-4482.

[161] C.E. Bronnimann, C.F. Ridenour, D.R. Kinney, G.E. Maciel, $2 \mathrm{D}{ }^{1} \mathrm{H}-{ }^{13} \mathrm{C}$ heteronuclear correlation spectra of representative organic solids, J. Magn. Reson., 97 (1992) 522-534. 
[162] P. Caravatti, G. Bodenhausen, R.R. Ernst, Heteronuclear solid-state correlation spectroscopy, Chemical Physical Letters, 89 (1982) 363-367.

[163] J.-D. Mao, K. Schmidt-Rohr, Absence of mobile carbohydrate domains in dry humic substances proven by NMR, and implications for organic - Contaminant sorption models, Environ. Sci. Technol., 40 (2006) 1751-1756.

[164] K.W. Zilm, G.G. Webb, Applications of zero field and 2-dimensional n.m.r. methods to fossil fuels, Fuel, 67 (1988) 707-713.

[165] J.D. Mao, R.L. Johnson, J. Lehmann, D.C. Olk, E.G. Neves, M.L. Thompson, K.

Schmidt-Rohr, Abundant and stable char residues in soils: Implications for soil fertility and carbon sequestration, Environ. Sci. Technol., 46 (2012) 9571-9576.

[166] X. Cao, C. Lattao, J.J. Pignatello, J. Mao, K. Schmidt-Rohr, Sorption selectivity in natural organic matter probed with fully deuterium-exchanged and carbonyl- ${ }^{13} \mathrm{C}$-labeled benzophenone and ${ }^{1} \mathrm{H}-{ }^{13} \mathrm{C}$ NMR spectroscopy, Environ. Sci. Technol., 48 (2014) 8645-8652. [167] P. Caravatti , P. Neuenschwander , R.R. Ernst, Charcterization of polymer blends by selective proton spin-diffusion NMR measurements, Macromolecules, 19 (1986) 1889-1895. [168] P. Caravatti , P. Neuenschwander , R.R. Ernst, Characterization of heterogeneous polymer blends by two-dimensional proton spin diffusion spectroscopy, Macromolecules, 18 (1985) 119-122.

[169] M. Goldman, L. Shen, Spin-spin relaxation in LaF3, Phys. Rev., 144 (1966) 321-331. [170] W.G. Hu, J.D. Mao, B.S. Xing, K. Schmidt-Rohr, Poly(methylene) crystallites in humic substances detected by nuclear magnetic resonance, Environ. Sci. Technol., 34 (2000) 530-534. [171] J. Jeener, B.H. Meier, T. Bachmann, R.R. Ernest, Investigation of exchange processes by two - dimensional NMR spectroscopy, J. Chem. Phys., 71 (1979) 4546-4553

[172] A.F. de Jong, A.P.M. Kentgens, W.S. Veeman, Two-dimensional exchange NMR in rotating solids: A technique to study very slow molecular reorientations, Chem. Phys. Lett., 109 (1984) 337-342.

[173] D.P. Burum, Combined rotation and multiple pulse spectroscopy (CRAMPS), Concepts Magn. Reson., 2 (1990) 213-227.

[174] P.G. Hatcher, Dipolar-dephasing ${ }^{13} \mathrm{C}$ NMR studies of decomposed wood and coalified xylem tissue: evidence for chemical structural changes associated with defunctionalization of lignin structural units during coalification, Energy Fuels, 2 (1988) 48-58.

[175] K. Schmidt-Rohr, J.D. Mao, Selective dephasing of $\mathrm{OH}$ and NH proton magnetization based on ${ }^{1} \mathrm{H}$ chemical-shift anisotropy recoupling, J. Magn. Reson., 157 (2002) 210-217. [176] A. Ramamoorthy, C. Wu, S. Opella, Three-dimensional solid-state NMR experiment that correlates the chemical shift and dipolar coupling frequencies of two heteronuclei, J. Magn. Reson., Ser B, 107 (1995) 88-90.

[177] J.Z. Song, P.A. Peng, Characterisation of black carbon materials by pyrolysis-gas chromatography-mass spectrometry, J. Anal. Appl. Pyrolysis, 87 (2010) 129-137.

[178] J. Mao, X. Fang, Y. Lan, A. Schimmelmann, M. Mastalerz, L. Xu, K. Schmidt-Rohr, Chemical and nanometer-scale structure of kerogen and its change during thermal maturation investigated by advanced solid-state ${ }^{13} \mathrm{C}$ NMR spectroscopy, Geochim. Cosmochim. Acta, 74 (2010) 2110-2127.

[179] B. Xing, J.J. Pignatello, Dual-mode sorption of low-polarity compounds in glassy poly (vinyl chloride) and soil organic matter, Environ. Sci. Technol., 31 (1997) 792-799. 
[180] W.J. Weber, P.M. McGinley, L.E. Katz, A distributed reactivity model for sorption by soils and sediments. 1. Conceptual basis and equilibrium assessments, Environ. Sci. Technol., 26 (1992) 1955-1962.

[181] Q. Chen, S. Hou, K. Schmidt-Rohr, A simple scheme for probehead background suppression in one-pulse ${ }^{1} \mathrm{H}$ NMR, Solid State Nucl. Magn. Reson., 26 (2004) 11-15. [182] K. Schmidt-Rohr, J. Clauss, H. Spiess, Correlation of structure, mobility, and morphological information in heterogeneous polymer materials by two-dimensional widelineseparation NMR spectroscopy, Macromolecules, 25 (1992) 3273-3277.

[183] E.R. deAzevedo, W.-G. Hu, T.J. Bonagamba, K. Schmidt-Rohr, Centerband-only detection of exchange: Efficient analysis of dynamics in solids by NMR, J. Am. Chem. Soc., 121 (1999) 8411-8412.

[184] S.A. Waksman, Humus Origin, Chemical Composition, and Importance in Nature, Williams and Wilkins, Baltimore, MD, 1936.

[185] M.a.M. Kononova, Soil organic matter; its nature, its role in soil formation and in soil fertility. Translated from the Russian by T.Z. Nowakowsli and A.C.D. Newman. , in, Pergamon Press, Oxford, UK., 1966.

[186] R. Haworth, The chemical nature of humic acid, Soil Sci., 111 (1971) 71-79.

[187] E.G. Gregorich, M.H. Beare, U.F. McKim, J.O. Skjemstad, Chemical and biological

characteristics of physically uncomplexed organic matter, Soil Sci. Soc. Am. J., 70 (2006) 975985.

[188] B.T. Christensen, Physical fractionation of soil and organic matter in primary particle size and density separates, Adv. Soil Sci., (1992) 1-90.

[189] J. Six, H. Bossuyt, S. Degryze, K. Denef, A history of research on the link between (micro) aggregates, soil biota, and soil organic matter dynamics, Soil Tillage Res., 79 (2004) 7 31.

[190] M.W. Schmidt, M.S. Torn, S. Abiven, T. Dittmar, G. Guggenberger, I.A. Janssens, M. Kleber, I. Kögel-Knabner, J. Lehmann, D.A. Manning, Persistence of soil organic matter as an ecosystem property, Nature, 478 (2011) 49-56.

[191] R. Swaby, J. Ladd, Chemical nature, microbial resistance, and origin of soil humus, in: G.J. Neale (Ed.) Transactions of Joint Meeting of Commissions IV and V, International Society of Soil Science, New Zealand Society of Soil Science Soil Bureau, Lower Hutt, N.Z., 1963, pp. 197-202.

[192] P. MacCarthy, The principles of humic substances, Soil Sci., 166 (2001) 738-751. [193] A.J. Simpson, M.J. Simpson, E. Smith, B.P. Kelleher, Microbially derived inputs to soil organic matter: are current estimates too low?, Environ. Sci. Technol., 41 (2007) 8070-8076. [194] M.F. Cotrufo, M.D. Wallenstein, C.M. Boot, K. Denef, E. Paul, The Microbial Efficiency - Matrix Stabilization (MEMS) framework integrates plant litter decomposition with soil organic matter stabilization: do labile plant inputs form stable soil organic matter?, Global Change Biol., 19 (2013) 988-995.

[195] J.M. Bremner, Organic Forms of Nitrogen, in: C.A. Black, D. Evans, R.C. Dinauer (Eds.) Methods of Soil Analysis, American Society of Agronomy, Madison, WI, 1965, pp. 1148-1178.

[196] D.S. Orlov, Humic substances of soils and general theory of humification, AA Balkema, Rotterdam, 1995. 
[197] H. Knicker, H.-D. Lüdemann, N-15 and C-13 CPMAS and solution NMR studies of N15 enriched plant material during 600 days of microbial degradation, Org. Geochem., 23 (1995) 329-341.

[198] H. Knicker, P.G. Hatcher, Survival of protein in an organic-rich sediment: possible protection by encapsulation in organic matter, Naturwissenschaften, 84 (1997) 231-234. [199] P. Leinweber, H.R. Schulten, Nonhydrolyzable forms of soil organic nitrogen: extractability and composition, J. Plant Nutr. Soil Sci., 163 (2000) 433-439.

[200] J.D. Mao, D.C. Olk, X.W. Fang, Z.Q. He, K. Schmidt-Rohr, Influence of animal manure application on the chemical structures of soil organic matter as investigated by advanced solidstate NMR and FT-IR spectroscopy, Geoderma, 146 (2008) 353-362.

[201] M.J. Simpson, Nuclear magnetic resonance based investigations of contaminant interactions with soil organic matter, Soil Sci. Soc. Am. J., 70 (2006) 995-1004.

[202] M. Thevenot, M.-F. Dignac, C. Rumpel, Fate of lignins in soils: a review, Soil Biol. Biochem., 42 (2010) 1200-1211.

[203] R. Benner, Chemical composition and reactivity, in: D.A. Hansell, C.A. Carlson (Eds.) Biogeochemistry of Marine Dissolved Organic Matter, Academic Press, San Diego, 2002, pp. 59-90.

[204] R. Benner, Molecular indicators of the bioavailability of dissolved organic matter, in: S.E. Findlay, R.L. Sinsabaugh (Eds.) Aquatic Ecosystems: Interactivity of Dissolved Organic Matter, Academic Press, San Diego, 2003, pp. 121-138.

[205] P. Sannigrahi, E.D. Ingall, R. Benner, Cycling of dissolved and particulate organic matter at station Aloha: Insights from C-13 NMR spectroscopy coupled with elemental, isotopic and molecular analyses, Deep-Sea Res. Part I-Oceanogr. Res. Pap., 52 (2005) 14291444.

[206] P.J. Mitchell, A.J. Simpson, M.J. Simpson, Dissolved organic matter, eMagRes, (2013) 503-516.

[207] R. Benner, J.D. Pakulski, M. McCarthy, J.I. Hedges, P.G. Hatcher, Bulk chemical characteristics of dissolved organic matter in the ocean., Science, 255 (1992) 1561-1564. [208] J.I. Hedges, P.G. Hatcher, J.R. Ertel, K.J. Meyers-Schulte, A comparison of dissolved humic substances from seawater with Amazon River counterparts by ${ }^{13} \mathrm{C}-\mathrm{NMR}$ spectrometry, Geochim. Cosmochim. Acta, 56 (1992) 1753-1757.

[209] D.M. McKnight, R. Harnish, R.L. Wershaw, J.S. Baron, S. Schiff, Chemical characteristics of particulate, colloidal, and dissolved organic material in Loch Vale Watershed, Rocky Mountain National Park, Biogeochemistry, 36 (1997) 99-124. [210] X. Zang, J.D.H. van Heemst, K.J. Dria, P.G. Hatcher, Encapsulation of protein in humic acid from a histosol as an explanation for the occurrence of organic nitrogen in soil and sediment, Org. Geochem., 31 (2000) 679-695.

[211] E. Kaiser, A.J. Simpson, K.J. Dria, B. Sulzberger, P.G. Hatcher, Solid-state and multidimensional solution-state NMR of solid phase extracted and ultrafiltered riverine dissolved organic matter, Environ. Sci. Technol., 37 (2003) 2929-2935.

[212] A. Brown, D.M. McKnight, Y.P. Chin, E.C. Roberts, M. Uhle, Chemical characterization of dissolved organic material in Pony Lake, a saline coastal pond in Antarctica, Mar. Chem., 89 (2004) 327-337.

[213] T.S. Bianchi, T. Filley, K. Dria, P.G. Hatcher, Temporal variability in sources of dissolved organic carbon in the lower Mississippi River, Geochim. Cosmochim. Acta, 68 (2004) 959-967. 
[214] R.L. Fimmen, R.M. Cory, Y.P. Chin, T.D. Trouts, D.M. McKnight, Probing the oxidation-reduction properties of terrestrially and microbially derived dissolved organic matter, Geochim. Cosmochim. Acta, 71 (2007) 3003-3015.

[215] V.I. Esteves, M. Otero, A.C. Duarte, Comparative characterization of humic substances from the open ocean, estuarine water and fresh water Org, Geochem., 40 (2009) 942-950. [216] H.A.N. Abdulla, E.C. Minor, R.F. Dias, P.G. Hatcher, Changes in the compound classes of dissolved organic matter along an estuarine transect: A study using FTIR and C-13 NMR, Geochim. Cosmochim. Acta, 74 (2010) 3815-3838.

[217] R. Jaffé, Y. Yamashita, N. Maie, W.T. Cooper, T. Dittmar, W.K. Dodds, J.B. Jones, T. Myoshi, J.R. Ortiz-Zayas, D.C. Podgorski, A. Watanabe, Dissolved organic matter in headwater streams: Compositional variability across climatic regions of North America, Geochim. Cosmochim. Acta, 94 (2012) 95-108.

[218] J.D. Mao, X.Q. Kong, K. Schmidt-Rohr, J.J. Pignatello, E.M. Perdue, Advanced solidstate NMR characterization of marine dissolved organic matter isolated using the coupled reverse osmosis/electrodialysis method, Environ. Sci. Technol., 46 (2012) 5806-5814. [219] M.A. Wilson, A.H. Gillam, P.J. Collin, Analysis of the structure of dissolved marine humic substances and their phytoplanktonic precursors by ${ }^{1} \mathrm{H}$ and ${ }^{13} \mathrm{C}$ nuclear magnetic resonance, Chem. Geol., 40 (1983) 187-201.

[220] E. Engelhaupt, T.S. Bianchi, Sources and composition of high - molecular - weight dissolved organic carbon in a southern Louisiana tidal stream (Bayou Trepagnier), Limnol. Oceanogr., 46 (2001) 917-926.

[221] J. Templier, S. Derenne, J.P. Croue, C. Largeau, Comparative study of two fractions of riverine dissolved organic matter using various analytical pyrolytic methods and a C-13 CP/MAS NMR approach, Org. Geochem., 36 (2005) 1418-1442.

[222] J. Templier, F. Miserque, N. Barré, F. Mercier, J.-P. Croué, S. Derenne, Is nitrogen functionality responsible for contrasted responses of riverine dissolved organic matter in pyrolysis?, J. Anal. Appl. Pyrolysis, 97 (2012) 62-72.

[223] J.F. Koprivnjak, P.H. Pfromm, E. Ingall, T.A. Vetter, P. Schmitt-Kopplin, N. Hertkorn, M. Frommberger, H. Knicker, E.M. Perdue, Chemical and spectroscopic characterization of marine dissolved organic matter isolated using coupled reverse osmosis-electrodialysis, Geochim. Cosmochim. Acta, 73 (2009) 4215-4231.

[224] Y. Zhang, W. Huang, Y. Ran, J. Mao, Compositions and constituents of freshwater dissolved organic matter isolated by reverse osmosis, Mar. Pollut. Bull., 85 (2014) 60-66. [225] U.G. Nwosu, R.L. Cook, ${ }^{13} \mathrm{C}$ Nuclear magnetic resonance and electron paramagnetic spectroscopic comparison of hydrophobic acid, transphilic acid, and reverse osmosis May 2012 isolates of organic matter from the Suwannee River, Environ. Eng. Sci., 32 (2015) 14-22. [226] R.G. Wetzel, P.G. Hatcher, T.S. Bianchi, Natural photolysis by ultraviolet irradiance of recalcitrant dissolved organic matter to simple substrates for rapid bacterial metabolism, Limnol. Oceanogr., 40 (1995) 1369-1380.

[227] M. Kulovaara, N. Corin, P. Backlund, J. Tervo, Impact of UV 254-radiation on aquatic humic substances, Chemosphere, 33 (1996) 783-790.

[228] C. Osburn, D. Morris, K. Thorn, R. Moeller, Chemical and optical changes in freshwater dissolved organic matter exposed to solar radiation, Biogeochemistry, 54 (2001) 251-278. [229] J.R. Helms, J. Mao, A. Stubbins, K. Schmidt-Rohr, R.G. Spencer, P.J. Hernes, K. Mopper, Loss of optical and molecular indicators of terrigenous dissolved organic matter during long-term photobleaching, Aquat. Sci., (2014) 353-373. 
[230] J.R. Helms, J. Mao, K. Schmidt-Rohr, H. Abdulla, K. Mopper, Photochemical flocculation of terrestrial dissolved organic matter and iron, Geochim. Cosmochim. Acta, 121 (2013) 398-413.

[231] Z.F. Liu, J.D. Mao, M.L. Peterson, C. Lee, S.G. Wakeham, P.G. Hatcher, Characterization of sinking particles from the northwest Mediterranean Sea using advanced solid-state NMR, Geochim. Cosmochim. Acta, 73 (2009) 1014-1026.

[232] J. Mao, L. Tremblay, J.-P. Gagné, Structural changes of humic acids from sinking organic matter and surface sediments investigated by advanced solid-state NMR: Insights into sources, preservation and molecularly uncharacterized components, Geochim. Cosmochim. Acta, 75 (2011) 7864-7880.

[233] J. Hwang, E.R. Druffel, T.I. Eglinton, D.J. Repeta, Source(s) and cycling of the nonhydrolyzable organic fraction of oceanic particles, Geochim. Cosmochim. Acta, 70 (2006) 5162-5168.

[234] J.I. Hedges, J.A. Baldock, Y. Gélinas, C. Lee, M. Peterson, S.G. Wakeham, Evidence for non-selective preservation of organic matter in sinking marine particles, Nature, 409 (2001) 801-804.

[235] M. McCarthy, T. Pratum, J. Hedges, R. Benner, Chemical composition of dissolved organic nitrogen in the ocean, Nature, 390 (1997) 150-154.

[236] L.I. Aluwihare, D.J. Repeta, S. Pantoja, C.G. Johnson, Two chemically distinct pools of organic nitrogen accumulate in the ocean, Science, 308 (2005) 1007-1010.

[237] N. Maie, K.J. Parish, A. Watanabe, H. Knicker, R. Benner, T. Abe, K. Kaiser, R. Jaffe, Chemical characteristics of dissolved organic nitrogen in an oligotrophic subtropical coastal ecosystem, Geochim. Cosmochim. Acta, 70 (2006) 4491-4506.

[238] L.I. Aluwihare, T. Meador, Chemical composition of marine dissolved organic nitrogen, in, San Diego, CA, USA: Academic Press, 2008, pp. 95-139.

[239] L.L. Clark, E.D. Ingall, R. Benner, Marine phosphorus is selectively remineralized, Nature, 393 (1998) 426-426.

[240] L.L. Clark, E.D. Ingall, R. Benner, Marine organic phosphorus cycling: Novel insights from nuclear magnetic resonance Am. J. Sci., 299 (1999) 724-737.

[241] L.C. Kolowith, E.D. Ingall, R. Benner, Composition and cycling of marine organic phosphorus, Limnol. Oceanogr., 46 (2001) 309-320.

[242] P. Sannigrahi, E.D. Ingall, R. Benner, Nature and dynamics of phosphorus-containing components of marine dissolved and particulate organic matter, Geochim. Cosmochim. Acta, 70 (2006) 5868-5882.

[243] D.M. Karl, K.M. Björkman, Chapter 5 - Dynamics of Dissolved Organic Phosphorus, in: D.A.H.A. Carlson (Ed.) Biogeochemistry of Marine Dissolved Organic Matter (Second Edition), Academic Press, Boston, 2014, pp. 233-334.

[244] C.L. Young, E.D. Ingall, Marine Dissolved Organic Phosphorus Composition: Insights from Samples Recovered Using Combined Electrodialysis/Reverse Osmosis, Aquat.

Geochem., 16 (2010) 563-574.

[245] J.S.P. Seinfeld, Atmospheric Chemistry and Physics: From Air Pollution to Climate Change, 2nd ed. ed., John Wiley \& Sons, Inc., Hoboken, New Jersey, 1998.

[246] M.-C.K. Chalbot, I., Nuclear magnetic resonance spectroscopy for determining the functional content of organic aerosols: A review, Environ. Pollut., 191 (2014) 232-249. 
[247] R.J.J. Park, D.J.; Humar, N. Yantosca, R.M., Regional visibility statistics in the United States: Natural and transboundary pollution influences, and implications for the Regional Haze Rule, Atmos. Environ., 40 (2006) 5405-5423.

[248] A. Zanobetti, M. Franklin, P. Koutrakis, J. Schwartz, Fine particulate air pollution and its components in association with cause-specific emergency admissions, Environ. Health, 8 (2009) 58.

[249] Y. Subbalakshmi, A.F. Patti, G.S.H. Lee, M.A. Hooper, Structural characterisation of macromolecularorganic material in air particulate matter using Py-GC-MS and solid state ${ }^{13} \mathrm{C}$ NMR, J. Environ. Monit., 2 (2000) 561-565.

[250] R.M. Duarte, A.C. Duarte, Atmospheric organic matter, eMagRes, 2 (2013) 415-426.

[251] R.M. Duarte, A.C. Duarte, Unraveling the structural features of organic aerosols by NMR spectroscopy: A review, Magn. Reson. Chem., 53 (2015) 658-666.

[252] R.M.B.O. Duarte, C.A. Pio, A.C. Duarte, Spectroscopic study of the water soluble organic matter isolated from atmospheric aerosols collected under different atmospheric conditions., Anal. Chim. Acta, 530 (2005) 7-14.

[253] R. Duarte, M,B.O., E.B.H. Santos, C.A. Pio, D. A.C., Comparison of structural features of water-soluble organic matter from atmospheric aerosols with those of aquatic humic substances, Atmos. Environ., 41 (2007) 8100-8113.

[254] P. Sannigrahi, A.P. Sullian, R.J. Webber, E.D. Ingall, Characterization of water-soluble organic carbon in urban atmospheric aerosols using solid-state NMR spectroscopy, Environ. Sci. Technol., 40 (2006) 666-672.

[255] J. Zhao, P. Peng, J. Song, S. Ma, G. Sheng, J. Fu, Characterization of macromolecular organic matter in atmospheric dust from Guangzhou, China, Atmos. Environ., 45 (2011) 56125620.

[256] M.S. Solum, A.F. Sarofim, R.J. Pugmire, T.H. Fletcher, H.F. Zhang, ${ }^{13}$ C NMR analysis of soot produced from model compounds and a coal, Energy Fuels, 15 (2001) 961-971.

[257] M.S. Solum, J.M. Veranth, Y. Jiang, A.M. Orendt, A.F. Sarofim, R.J. Pugmire, The study of anthracene aerosols by solid-state NMR and ESR, Energy Fuels, 17 (2003) 738-743. [258] J.R. Cronin, S. Chang, Organic matter in meteorites: molecular and isotopic analysis of the Murchison meteorite, in: J.M. Greenberg, C.X. mendoza-Gomez, V. Pirronello (Eds.) The Chemistry of Life's Origins, Kluwer Academic Publishers, Boston, MA, 1993, pp. 209-258. [259] R.A. Hayatsu, E. Anders, Organic compounds in meteorites and their origins, Top. Curr. Chem., 99 (1981) 1-37.

[260] J.R. Cronin, S. Pizzarello, D.P. Cruikshank, Organic matter in carbonaceous chondrites, planetary sattellites, asteroids and comets, in: J.F.K.a.M.S. Matthews (Ed.) Meteorites and the Early Solar System, The University of Arizona Press, Tucson, AZ, 1988, pp. 819-857.

[261] J.M. Hayes, Organic constituents of meteorites - A review, Geochim. Cosmochim. Acta, 31 (1967) 1395-1440.

[262] C.M.O.D. Alexander, S.S. S.S. Russell, J.W. Arden, R.D. Ash, M.M. Grady, C.T. Pillinger, The origin of chrondritic macromolecular organic matter: A carbon and nitrogen isotope study, Meteorit. Planet. Sci., 33 (1998) 603-622.

[263] G.J. Flynn, L.P. Keller, M. Feser, S. Wirick, C. Jacobsen, The origin of organic matter in the Solar system: Evidence from the interplanetary dust particles, Geochim. Cosmochim. Acta, 67 (2003) 4791-4806. 
[264] M.A. Stephton, C.T. Pillinger, L. Gilmour, Aromatic moieties in meteoritic macromolecular materials: Analysis by hydrolysis and d13 of individual compounds, Geochim. Cosmochim. Acta, 64 (2000) 321-328.

[265] G. Flynn, L.P. Keller, C. Jacobsen, S. Wirick, An assessment of the amount and types of organic matter contributed to the Earth by interplanetary dust, Adv. Space Res., 33 (2004) 5766.

[266] G.D. Cody, C.M.O.D. Alexander, F. Tera, Solid-state $\left({ }^{1} \mathrm{H}\right.$ and $\left.{ }^{13} \mathrm{C}\right)$ nuclear magnetic resonance spectroscopy of insoluble organic residue in the Murchison meteorite: A selfconsistent quantitative analysis, Geochim. Cosmochim. Acta, 66 (2002) 1851-1865.

[267] G.D. Cody, C.M.O.D. Alexander, NMR studies of chemical structural variation of insoluble organic matter from different carbonaceous chondrite groups., Geochim.

Cosmochim. Acta, 69 (2005) 1085-1097.

[268] J.R. Cronin, S. Pizzarello, J. Frye, ${ }^{13} \mathrm{C}$ NMR spectroscopy of the insoluble carbon of cabonaceous chondrites., Geochim. Cosmochim. Acta, 51 (1987) 299-303.

[269] A. Gardinier, S. Derenne, F. Robert, F. Behar, C. Largeau, J. Maquet, Solid-state $\mathrm{CP} / \mathrm{MAS}{ }^{13} \mathrm{C}$ NMR of the insoluble organic matter of the Orgueil and Murchison meteorites: quantitative study, Earth. Planet. Sci. Lett., 184 (2000) 9-21.

[270] S. Pizzarello, Y. Huang, L. Becker, R.J. Poreda, R.A. Nieman, G. Cooper, M. Williams, The organic content of the Tagish Lake meteorite, Science, 293 (2001) 2236-2239.

[271] G.D. Cody, E. Heying, C.M.O. Alexander, L.R. Nittler, A.L. Kilcoyne, S.A. Sandford, R.M. Stroud, Establishing a molecular relationship between chondritic and cometary organic solids, Proc. Natl. Acad. Sci. U.S.A., 108 (2011) 19171-19176.

[272] Y. Kebukawa, A.L.D. Kilcoyne, G.D. Cody, Exploring the potential formation of organic solids in chondrites and comets through polymerization of interstellar formaldehyde, Astrophys. J., 771 (2013) 19.

[273] H. Yabuta, C.M.O. Alexander, M.L. Fogel, A.L.D. Kilcoyne, G.D. Cody, A molecular and isotopic study of the macromolecular organic matter of the ungrouped C2 WIS 91600 and its relationship to Tagish Lake and PCA 91008, Meteorit. Planet. Sci., 45 (2010) 1446-1460. [274] S. Pizzarello, S.K. Davidowski, G.P. Holland, L.B. Williams, Processing of meteoritic organic materials as a possible analog of early molecular evolution in planetary environments, Proc. Natl. Acad. Sci. U.S.A., 110 (2013) 15614-15619.

[275] C.M.O. Alexander, G.D. Cody, Y. Kebukawa, R. Bowden, M.L. Fogel, A.L.D. Kilcoyne, L.R. Nittler, C.D.K. Herd, Elemental, isotopic, and structural changes in Tagish Lake insoluble organic matter produced by parent body processes, Meteorit. Planet. Sci., 49 (2014) 503-525.

[276] L. Remusat, S. Derenne, F. Robert, H. Knicker, New pyrolytic and spectroscopic data on Orgueil and Murchison insoluble organic matter: A different origin than soluble?, Geochim. Cosmochim. Acta, 69 (2005) 3919-3932.

[277] A.D. Alekseev, E.V. Ul'yanova, NMR potentials for studying physical processes in fossil coals, Physics-Uspekhi, 48 (2005) 1161.

[278] V.J. Bartuska, G.E. Maciel, J. Schaefer, E.O. Stejskal, Prospects for carbon-13 nuclear magnetic resonance analysis of solid fossil-fuel materials, Fuel, 56 (1977) 354-358.

[279] P.G. Hatcher, Chemical structural models for coalified wood (vitrinite) in low rank coal, Org. Geochem., 16 (1990) 959-968.

[280] F.P. Miknis, Z.S. Jiao, D.B. Macgowan, R.C. Surdam, Solid-state NMR characterization of Mowry shale from the Powder River Basin Org. Geochem., 20 (1993) 339-347. 
[281] R.L. Patience, A.L. Mann, I.J.F. Poplett, Determination of molecular structure of kerogens using ${ }^{13} \mathrm{C}$ NMR spectroscopy: II. The effects of thermal maturation on kerogens from marine sediments Geochim. Cosmochim. Acta, 56 (1992) 2725-2742.

[282] C.E. Snape, D.E. Axelson, R.E. Botto, J.J. Delpuech, P. Tekely, B.C. Gerstein, M.

Pruski, G.E. Maciel, M.A. Wilson, Quantitative reliability of aromaticity and related measurements on coals by ${ }^{13} \mathrm{C}$ n.m.r. A debate, Fuel, 68 (1989) 547-560.

[283] M.S. Solum, R.J. Pugmire, D.M. Grant, ${ }^{13}$ C Solid-state NMR of Argonne premium coals, Energy Fuels, 3 (1989) 187-193.

[284] R. Suggate, W. Dickinson, Carbon NMR of coals: The effects of coal type and rank, Int. J. Coal Geol., 57 (2004) 1-22.

[285] M.A. Wilson, R.J. Pugmire, J. Karas, L.B. Alemany, W.R. Woolfenden, D.M. Grant, P.H. Given, Carbon distribution in coals and coal macerals by cross polarization magic angle spinning carbon-13 nuclear magnetic resonance spectrometry, Anal. Chem., 56 (1984) 933943.

[286] Z. Wei, X. Gao, D. Zhang, J. Da, Assessment of thermal evolution of kerogen geopolymers with their structural parameters measured by solid-state ${ }^{13} \mathrm{C}$ NMR spectroscopy, Energy Fuels, 19 (2005) 240-250.

[287] X. Cao, J. Yang, J. Mao, Characterization of kerogen using solid-state nuclear magnetic resonance spectroscopy: A review, Int. J. Coal Geol., 108 (2013) 83-90.

[288] J.Z. Hu, M.S. Solum, C.M.V. Taylor, R.J. Pugmire, D.M. Grant, Structural determination in carbonaceous solids using advanced solid state NMR techniques, Energy Fuels, 15 (2001) 14-22.

[289] N.K. Sethi, R.J. Pugmire, J.C. Facelli, D.M. Grant, Quantitative determination of different carbon types in fusinite and anthracite coals from carbon-13 nuclear magnetic resonance chemical shielding line-shape analysis, Anal. Chem., 60 (1988) 1574-1579. [290] X. Cao, J.E. Birdwell, M.A. Chappell, Y. Li, J.J. Pignatello, J. Mao, Characterization of oil shale, isolated kerogen, and postpyrolysis residues using advanced ${ }^{13} \mathrm{C}$ solid-state nuclear magnetic resonance spectroscopy, AAPG Bull., 97 (2013) 421-436.

[291] X. Cao, M.A. Chappell, A. Schimmelmann, M. Mastalerz, Y. Li, W. Hu, J. Mao, Chemical structure changes in kerogen from bituminous coal in response to dike intrusions as investigated by advanced solid-state ${ }^{13} \mathrm{C}$ NMR spectroscopy, Int. J. Coal Geol., 108 (2013) 5364.

[292] Y. Li, X. Cao, D. Zhu, M.A. Chappell, L.F. Miller, J. Mao, Characterization of coals and their laboratory-prepared black carbon using advanced solid-state ${ }^{13} \mathrm{C}$ nuclear magnetic resonance spectroscopy, Fuel Processing Technology, 96 (2012) 56-64.

[293] S.M. Althaus, K. Mao, G.J. Kennedy, M. Pruski, Solid-state NMR studies of fossil fuels using one-and two-dimensional methods at high magnetic field, Energy Fuels, 26 (2012) 44054412.

[294] K. Mao, M. Pruski, Directly and indirectly detected through-bond heteronuclear correlation solid-state NMR spectroscopy under fast MAS, J. Magn. Reson., 201 (2009) 165174.

[295] J.-D. Mao, L.S. Hundal, K. Schmidt-Rohr, M.L. Thompson, Nuclear magnetic resonance and diffuse-reflectance infrared Fourier-transform spectroscopy of sludge-derived biocolloidal organic matter, Environ. Sci. Technol., 37 (2003) 1751-1757.

[296] Ü. Lille, I. Heinmaa, T. Pehk, Molecular model of Estonian kukersite kerogen evaluated by ${ }^{13}$ C MAS NMR spectra, Fuel, 82 (2003) 799-804. 
[297] J.D. Mao, A. Schimmelmann, M. Mastalerz, P.G. Hatcher, Y. Li, Structural features of a Bituminous coal and their changes during low-temperature oxidation and loss of volatiles investigated by advanced solid-state NMR spectroscopy, Energy Fuels, 24 (2010) 2536-2544. 


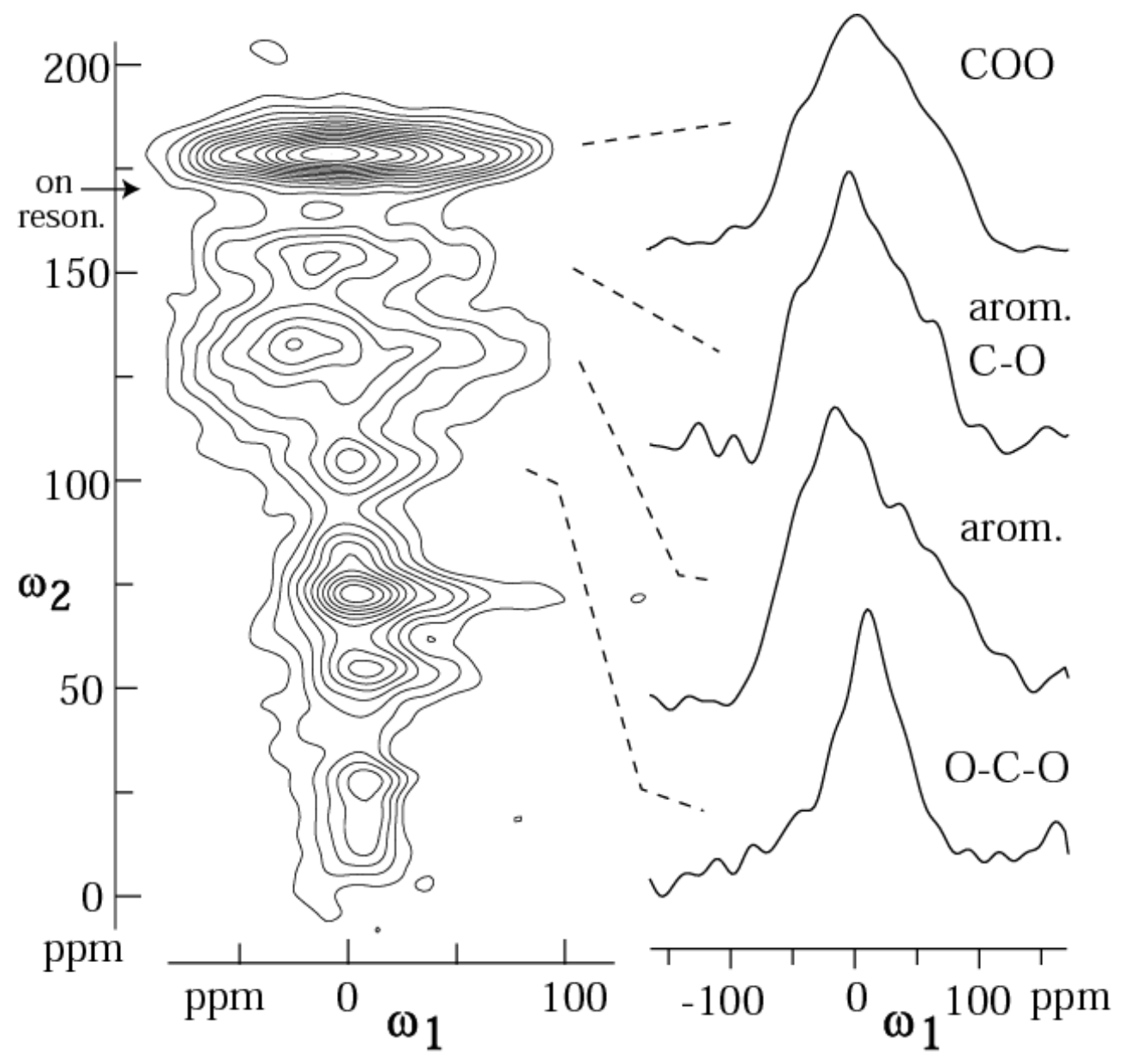

